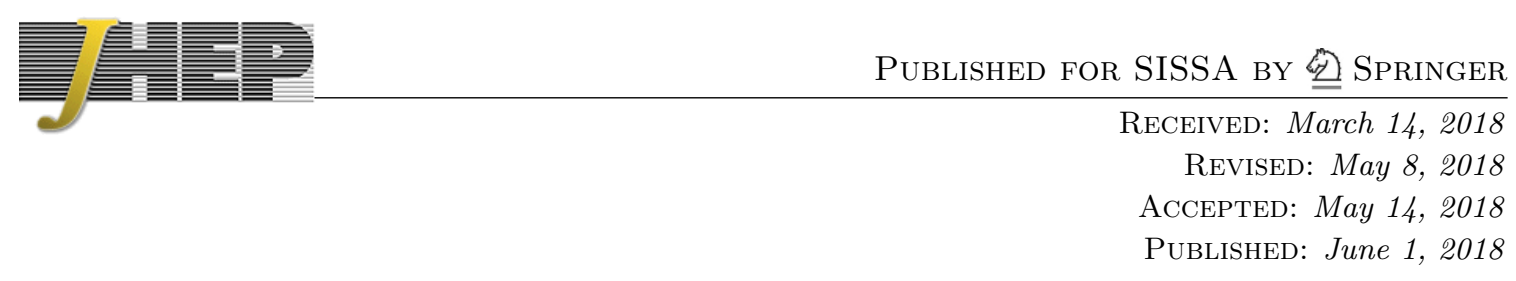

\title{
Lepton flavour violation in the MSSM: exact diagonalization vs mass expansion
}

\author{
Andreas Crivellin, ${ }^{a}$ Zofia Fabisiewicz, ${ }^{b}$ Weronika Materkowska, ${ }^{b}$ Ulrich Nierste, ${ }^{c}$ \\ Stefan Pokorski ${ }^{b}$ and Janusz Rosiek ${ }^{b}$ \\ a Paul Scherrer Institut, \\ CH-5232 Villigen PSI, Switzerland \\ ${ }^{b}$ Faculty of Physics, University of Warsaw, \\ Pasteura 5, 02-093 Warsaw, Poland \\ ${ }^{c}$ Institut für Theoretische Teilchenphysik, Karlsruhe Institute of Technology, \\ 76128 Karlsruhe, Germany \\ E-mail: andreas.crivellin@cern.ch, zofia.fabisiewicz@fuw.edu.pl, \\ weronika.materkowska@fuw.edu.pl, ulrich.nierste@kit.edu, \\ stefan.pokorski@fuw.edu.pl, janusz.rosiek@fuw.edu.pl
}

ABSTRACT: The forthcoming precision data on lepton flavour violating (LFV) decays require precise and efficient calculations in New Physics models. In this article lepton flavour violating processes within the Minimal Supersymmetric Standard Model (MSSM) are calculated using the method based on the Flavour Expansion Theorem, a recently developed technique performing a purely algebraic mass-insertion expansion of the amplitudes. The expansion in both flavour-violating and flavour-conserving off-diagonal terms of sfermion and supersymmetric fermion mass matrices is considered. In this way the relevant processes are expressed directly in terms of the parameters of the MSSM Lagrangian. We also study the decoupling properties of the amplitudes. The results are compared to the corresponding calculations in the mass eigenbasis (i.e. using the exact diagonalization of the mass matrices). Using these methods, we consider the following processes: $\ell \rightarrow \ell^{\prime} \gamma$, $\ell \rightarrow 3 \ell^{\prime}, \ell \rightarrow 2 \ell^{\prime} \ell^{\prime \prime}, h \rightarrow \ell \ell^{\prime}$ as well as $\mu \rightarrow e$ conversion in nuclei. In the numerical analysis we update the bounds on the flavour changing parameters of the MSSM and examine the sensitivity to the forthcoming experimental results. We find that flavour violating muon decays provide the most stringent bounds on supersymmetric effects and will continue to do so in the future. Radiative $\ell \rightarrow \ell^{\prime} \gamma$ decays and leptonic three-body decays $\ell \rightarrow 3 \ell^{\prime}$ show an interesting complementarity in eliminating "blind spots" in the parameter space. In our analysis we also include the effects of non-holomorphic $A$-terms which are important for the study of LFV Higgs decays.

KeYwORDS: Supersymmetry Phenomenology

ARXIV EPRINT: 1802.06803 


\section{Contents}

1 Introduction 1

2 Effective LFV interactions 2

$2.1 \gamma-\ell-\ell^{\prime}$ interactions 2

$2.2 Z-\ell-\ell^{\prime}$ interactions 4

2.3 LFV Higgs interactions 5

2.4 Box contributions 7

2.4.1 Leptonic operators with $J \neq K$ and $I \neq L \quad 8$

2.4.2 Leptonic operators with $J=K$ and $I \neq L \quad 8$

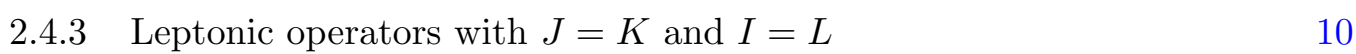

$\begin{array}{lll}2.4 .4 & \text { Operators with two leptons and two quarks } & 10\end{array}$

3 Observables 10

$\begin{array}{lll}3.1 & \text { Radiative lepton decays: } \ell^{I} \rightarrow \ell^{J} \gamma & 10\end{array}$

$3.2 h(H) \rightarrow \bar{\ell}^{I} \ell^{J}$ decays 11

$3.3 \ell^{I} \rightarrow \ell^{J} \ell^{K} \bar{\ell}^{L}$ decays 11

$3.4 \mu \rightarrow e$ conversion in nuclei 13

4 Mass eigenstates vs. mass insertions calculations 16

5 Phenomenological analysis $\quad 21$

5.1 Generic bounds on LFV parameters 21

5.2 Dependence on the mass splitting 24

$\begin{array}{lll}5.3 & \text { Correlations between LFV processes } & 28\end{array}$

5.4 Non-decoupling effects in LFV Higgs decays 29

6 Conclusions $\quad 32$

$\begin{array}{ll}\text { A MSSM Lagrangian and vertices } & 33\end{array}$

B Loop integrals $\quad 36$

$\begin{array}{ll}\text { C Divided differences } & 37\end{array}$

$\begin{array}{ll}\text { D Box diagrams in the mass eigenstates basis } & 38\end{array}$

E Effective lepton couplings in the leading MI order $\quad 41$

$\begin{array}{lll}\text { E.1 Lepton-photon vertex } & 41\end{array}$

E.1.1 Tensor (magnetic) couplings $\quad 41$

$\begin{array}{lll}\text { E.1.2 Vector couplings } & 42\end{array}$

E.2 Lepton- $Z^{0}$ vertex 43

E.3 CP-even Higgs-lepton vertex 45

$\begin{array}{lll}\text { E.4 CP-odd Higgs-lepton vertex } & 47\end{array}$

$\begin{array}{lll}\text { E.5 4-lepton box diagrams } & 47\end{array}$ 


\section{Introduction}

So far, the LHC did not observe any particles beyond those of the Standard Model (SM). Complementary to direct high energy searches at the LHC, there is a continuous effort in indirect searches for new physics (NP). In this respect, a promising approach is the search for processes which are absent - or extremely suppressed - in the SM such as lepton flavour violation (LFV) which is forbidden in the SM in the limit of vanishing neutrino masses. The experimental sensitivity for rare LFV processes such as $\ell \rightarrow \ell^{\prime} \gamma, \mu \rightarrow e$ conversion in nuclei and $\ell \rightarrow \ell^{\prime} \mu^{+} \mu^{-}$or $\ell \rightarrow \ell^{\prime} e^{+} e^{-}$will improve significantly in the near future, probing scales well beyond those accessible at foreseeable colliders. Furthermore, the discovery of the $125 \mathrm{GeV}$ Higgs boson $h[1,2]$ has triggered an enormous experimental effort in measuring its properties, including studies of its LFV decays. The most recent experimental limits on the LFV processes are given in table 2 in section 5 .

Many studies of LFV processes within the MSSM (and possible extensions of it) exist (see e.g. refs. [3-29] and ref. [30] for a recent review). In this article we revisit this subject in the light of the new calculational methods which have been recently developed [31, 32]. These methods allow for a systematic expansion of the amplitudes of the LFV processes in terms of mass insertions (MI), i.e. in terms of off-diagonal elements of the mass matrices. We show that a transparent qualitative behaviour of the amplitudes of the LFV processes is obtained by expanding them not only in the flavour-violating off-diagonal terms in the sfermion mass matrices but also in the flavour conserving but chirality violating entries related to the tri-linear $A$-terms as well as in the off-diagonal terms of the gaugino and higgsino mass matrices. This procedure is useful because in the MI approximation we work directly with the parameters of the Lagrangian and can therefore easily put experimental bounds on them. We compare the results of the calculations performed in the mass eigenbasis (i.e. using a numerical diagonalization of the slepton mass matrices) with those obtained at leading non-vanishing order of the MI approximation, in different regions of the supersymmetric parameter space and considering various decoupling limits. Of course, the MI approximation $[33,34]$ has already been explored for many years as a very useful tool in flavour physics. However, a detailed comparison between the full calculation and the MI approximation is still lacking, partly because a fully systematic discussion of the MI approximation [31] to any order and the technical tools facilitating it [32] have not been available until recently.

Concerning the phenomenology, we summarise and update the bounds on the flavour violating SUSY parameters, show their complementarity and examine the impact of the anticipated increase in the experimental sensitivity. We investigate in detail the decay $h \rightarrow \mu \tau$ showing the results in various decoupling limits and analyse the role of the socalled non-holomorphic $A$-terms [35-42], which are usually neglected in literature. We also avoid simplifying assumptions on the sparticle spectrum and assume neither degeneracies nor hierarchies among the supersymmetric particles.

This article is structured as follows: in section 2 we establish our conventions and present the results for the 2-point, 3-point, and 4-point functions related to flavour violating charged lepton interactions in the mass eigenbasis, i.e. expressed in terms of rotation 
matrices and physical masses. Section 3 contains the formula for the decay rates of the processes under investigation. In section 4 we discuss the MI expansion and summarise important properties of the decoupling limits $M_{\mathrm{SUSY}} \rightarrow \infty$ and $M_{A} \rightarrow \infty$. In section 5 we present the numerical bounds on LFV parameters obtained from current experimental measurements and discuss the dependence of the results on the SUSY spectrum. We also discuss the correlations between the radiative decays and the 3-body decays of charged lepton as well as the non-decoupling effects in LFV neutral Higgs decays. Finally we conclude in section 6. All required Feynman rules used in our calculations are collected in appendix A. The definitions of loop integrals can be found in appendix B. In appendix $\mathrm{C}$ we explain the notation for the "divided differences" of the loop functions used in the expanded form of the amplitudes. The expression for the 4-lepton box diagrams and for the MI-expanded expression of the amplitudes are given in the appendices D and E, respectively.

\section{Effective LFV interactions}

In this section we collect the analytical formula in the mass eigenbasis for flavour violating interactions generated at the one-loop level. ${ }^{1}$ We use the notation and conventions for the MSSM as given in refs. [43, 44]. ${ }^{2}$

In our analysis, we include the so-called non-holomorphic trilinear soft SUSY breaking terms:

$$
L_{n h}=\sum_{I, J=1}^{3} \sum_{i=1}^{2}\left(A_{l}^{\prime I J} H_{i}^{2 \star} L_{i}^{I} R^{J}+A_{d}^{\prime I J} H_{i}^{2 \star} Q_{i}^{I} D^{J}+A_{u}^{\prime I J} H_{i}^{1 \star} Q_{i}^{I} U^{J}+\text { H.c. }\right),
$$

which couple up(down)-sfermions to the down(up)-type Higgs doublets. Here, as throughout the rest of the paper, capital letters $I, J=1,2,3$ denote flavour indices and the small letters $i=1,2$ are $\mathrm{SU}(2)_{L}$ indices.

\section{$2.1 \gamma-\ell-\ell^{\prime}$ interactions}

We define the effective Lagrangian for flavour violating couplings of leptons to on-shell photons as

$$
L_{\ell \gamma}=-e \sum_{I, J}\left(F_{\gamma}^{J I} \bar{\ell}^{J} \sigma_{\mu \nu} P_{L} \ell^{I}+F_{\gamma}^{I J *} \bar{\ell}^{J} \sigma_{\mu \nu} P_{R} \ell^{I}\right) F^{\mu \nu}
$$

The SM contribution to $F_{\gamma}^{J I}$ is suppressed by powers of $m_{\nu}^{2} / M_{W}^{2}$ and thus completely negligible. In the mass eigenbasis the supersymmetric contributions to $F_{\gamma}^{J I}$ come from the diagrams displayed in figure 1 . Let us decompose $F_{\gamma}$ in the following way

$$
F_{\gamma}^{J I}=F_{\gamma A}^{J I}-m_{J} F_{\gamma L B}^{J I}-m_{I} F_{\gamma R B}^{J I}
$$

\footnotetext{
${ }^{1}$ Note that these expressions are not valid in the flavour conserving case where additional terms should be included and renormalization is required.

${ }^{2}$ The conventions of $[43,44]$ are very similar to the later introduced and now widely accepted SLHA2 [45] notation, up to the minor differences summarised in the appendix A.
} 

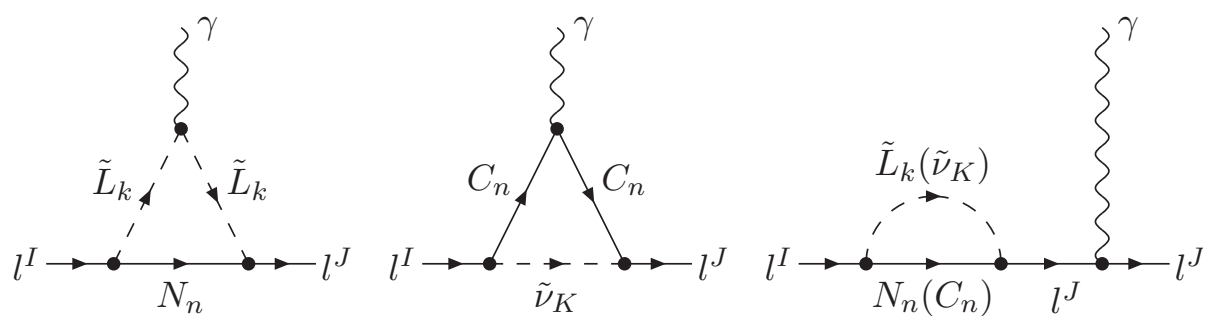

Figure 1. One-loop supersymmetric contributions to the LF violating effective lepton-photon interaction (mirror-reflected self-energy diagram not shown).

with

$$
\begin{aligned}
(4 \pi)^{2} F_{\gamma A}^{J I}= & \sum_{K=1}^{3} \sum_{n=1}^{2} V_{\ell \tilde{\nu} C, R}^{J K n *} V_{\ell \tilde{\nu} C, L}^{I K n} m_{C_{n}} C_{11}\left(m_{C_{n}}, m_{\tilde{\nu}_{K}}\right) \\
& -\frac{1}{2} \sum_{k=1}^{6} \sum_{n=1}^{4} V_{\ell \tilde{L} N, R}^{J k n *} V_{\ell \tilde{L} N, L}^{I K n} m_{N_{n}} C_{12}\left(m_{\tilde{L}_{k}}, m_{N_{n}}\right) \\
(4 \pi)^{2} F_{\gamma L B}^{J I}= & -\sum_{K=1}^{3} \sum_{n=1}^{2} V_{\ell \tilde{\nu} C, L}^{J K n *} V_{\ell \tilde{\nu} C, L}^{I K n} C_{23}\left(m_{C_{n}}, m_{\tilde{\nu}_{K}}\right) \\
& +\frac{1}{2} \sum_{k=1}^{6} \sum_{n=1}^{4} V_{\ell \tilde{L} N, L}^{J k n *} V_{\ell \tilde{L} N, L}^{I k n} C_{23}\left(m_{\tilde{L}_{k}}, m_{N_{n}}\right) .
\end{aligned}
$$

Here, $V$ abbreviates the tree-level lepton-slepton-neutrino and lepton-sneutrino-chargino vertices, i.e. the subscripts of $V$ stand for the interacting particles and the chirality of the lepton involved. The super-scripts refer to the lepton or slepton flavour as well as to the chargino and neutralino involved. The specific form of the chargino and neutralino vertices $V_{L(R)}$ is defined in appendix A and the 3-point loop functions $C_{i j}$ are given in appendix B. $F_{\gamma A}\left(F_{\gamma L B}\right)$ denotes the parts of the amplitude which is (not) proportional to the masses of fermions exchanged in the loop. $F_{\gamma R B}$ can be obtained from $F_{\gamma L B}$ by exchanging $L \leftrightarrow R$ on the r.h.s. of eq. (2.4).

Gauge invariance requires that LFV (axial) vectorial photon couplings vanish for onshell external particles. However, off-shell photon contributions are necessary to calculate three body decays of charged leptons. The vectorial part of the amplitude for the $\gamma \ell \ell^{\prime}$ vertex can be written as

$$
i A_{\gamma}^{J I \mu}=i e q^{2} \bar{u}_{J}\left(p_{J}\right)\left(\Gamma_{\gamma L}^{J I} P_{L}+\Gamma_{\gamma R}^{J I} P_{R}\right) \gamma^{\mu} u_{I}\left(p_{I}\right),
$$

where $q=p_{I}-p_{J}$ and $\Gamma_{\gamma L}^{J I}$ is at the leading order in $p^{2} / M_{\text {SUSY }}^{2}$ momentum independent and reads

$$
\begin{aligned}
\Gamma_{\gamma L}^{J I}= & \sum_{K=1}^{3} \sum_{n=1}^{2} V_{\ell \tilde{\nu} C, L}^{J K n *} V_{\ell \tilde{\nu} C, L}^{I K n} C_{01}\left(m_{C_{n}}, m_{\tilde{\nu}_{K}}\right) \\
& -\sum_{k=1}^{6} \sum_{n=1}^{4} V_{\ell \tilde{L} N, L}^{J k n *} V_{\ell \tilde{L} N, L}^{I k n} C_{02}\left(m_{N_{n}}, m_{\tilde{L}_{k}}\right)
\end{aligned}
$$



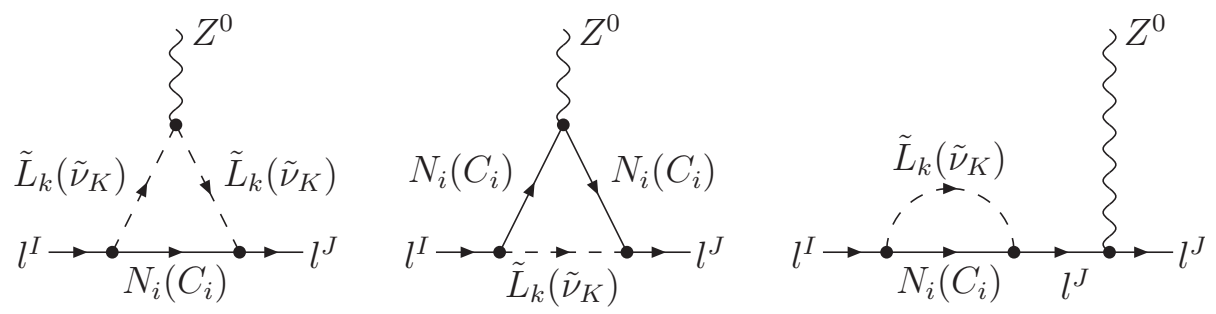

Figure 2. One-loop supersymmetric contributions to the LFV effective lepton- $Z^{0}$ interaction (the mirror-reflected self-energy diagram not shown).

$\Gamma_{\gamma R}^{J I}$ can be obtained by replacing $L \leftrightarrow R$. Again, the loop functions $C_{01}, C_{02}$ are defined in appendix B.

Finally, one should note that for heavy MSSM spectrum the 2-loop Barr-Zee diagrams [46] involving the non-decoupling LFV Higgs interactions (see section 5.4) are important and have to be included [47-52].

\section{$2.2 \quad Z-\ell-\ell^{\prime}$ interactions}

In order to calculate the three body decays of charged leptons as are considered in section 3.3 it is sufficient to calculate the effective $Z-\ell-\ell^{\prime}$ interactions in the limit of vanishing external momenta. The Wilson coefficients of the effective Lagrangian for the $Z$ coupling to charged leptons are generated at one-loop level by the diagrams shown in figure 2 and can be written as

$$
L_{\ell Z}^{J I}=\left(F_{Z L}^{J I} \bar{\ell}^{J} \gamma_{\mu} P_{L} \ell^{I}+F_{Z R}^{J I} \bar{\ell}^{J} \gamma_{\mu} P_{R} \ell^{I}\right) Z^{\mu}
$$

with

$$
\begin{aligned}
& F_{Z L}^{J I}=\Gamma_{Z L}^{J I}-\frac{e\left(1-2 s_{W}^{2}\right)}{2 s_{W} c_{W}} \Sigma_{V L}^{J I}(0) \\
& F_{Z R}^{J I}=\Gamma_{Z R}^{J I}+\frac{e s_{W}}{c_{W}} \Sigma_{V R}^{J I}(0)
\end{aligned}
$$

Here, $\Gamma_{Z L(R)}$ denote the contribution originating from the one-particle irreducible (1PI) vertex diagram and $\Sigma_{V L(R)}$ is the left-(right-)handed part of the lepton self-energy defined as

$$
\Sigma^{J I}\left(p^{2}\right)=\Sigma_{V L}^{J I}\left(p^{2}\right) \not p P_{L}+\Sigma_{V R}^{J I}\left(p^{2}\right) \not p P_{R}+\Sigma_{m L}^{J I}\left(p^{2}\right) P_{L}+\Sigma_{m R}^{J I}\left(p^{2}\right) P_{R}
$$

Contrary to the left- and right-handed magnetic photon-lepton couplings, which change chirality, the $Z \bar{\ell}^{I} \ell^{J}$ coupling is chirality conserving. Therefore, the Wilson coefficients of the left-handed and right-handed couplings are not related to each other but rather satisfy $F_{Z L(R)}^{I J}=F_{Z L(R)}^{J *}$. In the mass eigenbasis the vectorial part of the lepton self-energy and 
the 1PI triangle diagrams are given by (see appendix A for definitions of vertices $V$ )

$$
\begin{aligned}
(4 \pi)^{2} \Sigma_{V L}^{J I}\left(p^{2}\right)= & \sum_{i=1}^{2} \sum_{K=1}^{3} V_{\ell \tilde{\nu} C, L}^{I K i} V_{\ell \tilde{\nu} C, L}^{J K i *} B_{1}\left(p, m_{\tilde{\nu}_{K}}, m_{C_{i}}\right) \\
& +\sum_{i=1}^{4} \sum_{j=1}^{6} V_{\ell \tilde{L} N, L}^{I j i} V_{\ell \tilde{L} N, L}^{J j i *} B_{1}\left(p, m_{L_{j}}, m_{N_{i}}\right), \\
(4 \pi)^{2} \Gamma_{Z L}^{J I}= & \frac{1}{2} \sum_{i, j=1}^{2} \sum_{K=1}^{3} V_{\ell \tilde{\nu} C, L}^{I K i} V_{\ell \tilde{\nu} C, L}^{J K j *}\left(V_{C C Z, L}^{i j} C_{2}\left(m_{\tilde{\nu}_{K}}, m_{C_{i}}, m_{C_{j}}\right)\right. \\
& \left.-2 V_{C C Z, R}^{i j} m_{C_{i}} m_{C_{j}} C_{0}\left(m_{\tilde{\nu}_{K}}, m_{C_{i}}, m_{C_{j}}\right)\right) \\
& +\frac{e}{4 s W c} \sum_{i=1}^{2} \sum_{K=1}^{3} V_{\ell \tilde{\nu} C, L}^{I K i} V_{\ell \tilde{\nu} C, L}^{J K i *} C_{2}\left(m_{\tilde{\nu}_{K}}, m_{\tilde{\nu}_{K}}, m_{C_{i}}\right) \\
& +\frac{1}{2} \sum_{j=1}^{6} \sum_{i, k=1}^{4} V_{\ell \tilde{L} N, L}^{I j i} V_{\ell \tilde{L} N, L}^{J j k *}\left(V_{N N Z, L}^{i k} C_{2}\left(m_{L_{j}}, m_{N_{i}}, m_{N_{k}}\right)\right. \\
& \left.-2 V_{N N Z, R}^{i k} m_{N_{i}} m_{N_{k}} C_{0}\left(m_{L_{j}}, m_{N_{i}}, m_{N_{k}}\right)\right) \\
& -\frac{1}{2} \sum_{j, k=1}^{6} \sum_{i=1}^{4} V_{\ell \tilde{L} N, L}^{I j i} V_{\ell \tilde{L} N, L}^{J k i *} V_{L L Z}^{j k} C_{2}\left(m_{L_{j}}, m_{L_{k}}, m_{N_{i}}\right),
\end{aligned}
$$

at vanishing external momenta with obvious replacements $L \leftrightarrow R$ for $\Sigma_{V R}^{J I}, \Gamma_{Z R}^{J I}$.

\subsection{LFV Higgs interactions}

To compactify the notation, we denote the CP-even Higgs boson decays by $H_{0}^{K} \rightarrow \bar{\ell}^{I} \ell^{J}$, where, following again the notation of $[43,44], H \equiv H_{0}^{1}, h \equiv H_{0}^{2}$. As usual, we denote CP-odd neutral Higgs boson by $A_{0}$.

In order to study $h \rightarrow \ell \ell^{\prime}$ decays precisely, we keep the terms depending on the external Higgs mass. Therefore, we assume the following effective action governing the LFV Higgslepton interaction:

$$
\begin{aligned}
A_{H \mathrm{eff}}^{\ell}= & \bar{\ell}^{J}\left(k_{J}\right)\left(F_{h \ell}^{J I K}\left(k_{J}, k_{I}\right) P_{L}+F_{h \ell}^{I J K *}\left(k_{J}, k_{I}\right) P_{R}\right) \ell^{I}\left(k_{I}\right) H_{0}^{K}\left(k_{I}-k_{J}\right) \\
& +\bar{\ell}^{J}\left(k_{J}\right)\left(F_{A \ell}^{J I}\left(k_{J}, k_{I}\right) P_{L}+F_{A \ell}^{I J *}\left(k_{J}, k_{I}\right) P_{R}\right) \ell^{I}\left(k_{I}\right) A_{0}\left(k_{I}-k_{J}\right) .
\end{aligned}
$$

In addition, to calculate the $\mu \rightarrow e$ conversion rate one needs to include the effective Higgsquark couplings. For this purpose, one can set all external momenta to zero and consider the effective Lagrangian

$$
L_{H \mathrm{eff}}^{q}=\bar{u}^{J}\left(F_{h u}^{J K} P_{L}+F_{h u}^{I J K *} P_{R}\right) u^{I} H_{0}^{K}+\bar{d}^{J}\left(F_{h d}^{J I K} P_{L}+F_{h d}^{I J K *} P_{R}\right) d^{I} H_{0}^{K} .
$$

However, in this article we consider only the lepton sector and therefore do not give the explicit forms of Higgs quark couplings. The relevant 1-loop expressions in the same notation as used in the current paper are given in ref. [53] and the formulae that take into account also non-decoupling chirally enhanced corrections and 2-loop QCD corrections in the general MSSM can be found in refs. [54-56]. ${ }^{3}$

\footnotetext{
${ }^{3}$ Earlier accounts on chiral resummation can be found in refs. [57-65].
} 

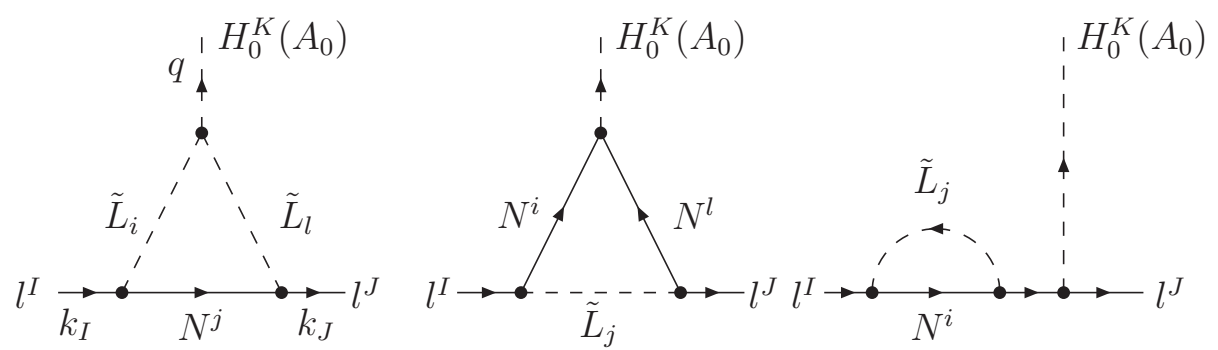

Figure 3. Slepton-neutralino diagrams contributing to the $H_{0}^{K} \rightarrow \ell^{I} \bar{\ell}^{J}$ and $A_{0} \rightarrow \ell^{I} \bar{\ell}^{J}$ decays in the MSSM (the mirror-reflected self-energy diagram is omitted).

At the 1-loop level there are eight diagrams contributing to the effective lepton Yukawa couplings. The ones with slepton and neutralino exchange are displayed in figure 3 , while diagrams with the chargino exchange can be obtained by the obvious replacements $N \rightarrow$ $C, L \rightarrow \tilde{\nu}$.

The expressions for $F_{h}$ and $F_{A}$ are obtained from 1PI triangle diagrams and the scalar part of lepton self-energies (see eq. (2.9)) while the chirality conserving parts of the selfenergies are absorbed by a field rotation required to go to the physical basis with a diagonal lepton mass matrix. Therefore,

$$
\begin{gathered}
F_{h}^{J I K}\left(k_{J}, k_{I}\right)=\Gamma_{h}^{J I K}\left(k_{J}, k_{I}\right)-\frac{Z_{R}^{1 K}}{v_{1}} \Sigma_{m L}^{J I}(0), \\
F_{A}^{J I}\left(k_{J}, k_{I}\right)=\Gamma_{A}^{J I}\left(k_{J}, k_{I}\right)-\frac{i \sin \beta}{v_{1}} \Sigma_{m L}^{J I}(0),
\end{gathered}
$$

where the $Z_{R}$ denotes the CP-even Higgs mixing matrix (see appendix A) and the scalar self-energy contributions are evaluated at zero momentum transfer and given by:

$$
\begin{aligned}
(4 \pi)^{2} \Sigma_{m L}^{J I}(0)= & \sum_{i=1}^{2} \sum_{L=1}^{3} m_{C_{i}} V_{\ell \tilde{\nu} C, L}^{I L i} V_{\ell \tilde{\nu} C, R}^{J L i *} B_{0}\left(0, m_{\tilde{\nu}_{L}}, m_{C_{i}}\right) \\
& +\sum_{i=1}^{4} \sum_{j=1}^{6} m_{N_{i}} V_{\ell \tilde{L} N, L}^{I j i} V_{\ell \tilde{L} N, R}^{J j i *} B_{0}\left(0, m_{L_{j}}, m_{N_{i}}\right)
\end{aligned}
$$

The neutralino-slepton contributions to the 1PI vertex diagrams can be written as (the symbols in square brackets denote common arguments of the 3-point functions) ${ }^{4}$

$$
\begin{aligned}
& (4 \pi)^{2} \Gamma_{h}^{J I K}\left(k_{J}, k_{I}\right)=-\sum_{n=1}^{4} \sum_{l, m=1}^{6} V_{\ell \tilde{L} N, L}^{J m n *} V_{\ell \tilde{L} N, L}^{I l n} V_{H \tilde{L} \tilde{L}}^{K l m} m_{N_{n}} C_{0}\left[k_{J}, k_{I}-k_{J}, m_{N_{n}}, m_{\tilde{L}_{m}}, m_{\tilde{L}_{l}}\right] \\
& -\sum_{l, n=1}^{4} \sum_{m=1}^{6} V_{\ell \tilde{L} N, R}^{J n m *} V_{\ell \tilde{L} N, L}^{I n l}\left(V_{N H N, R}^{l K m} C_{2}+V_{N H N, L}^{l K m} m_{N_{l}} m_{N_{m}} C_{0}\right)\left[k_{J}, k_{I}-k_{J}, m_{\tilde{L}_{n}}, m_{N_{m}}, m_{N_{l}}\right],
\end{aligned}
$$

\footnotetext{
${ }^{4}$ As we shall see later using MI expanded formulae (see appendix E.3), due to strong cancellations the leading order terms in eqs. (2.15), (2.16) are suppressed by the ratios of $m_{\ell} / M_{W}$ or $A_{l}^{\prime} / M_{\mathrm{SUSY}}$. Additional terms linear in $m_{\ell} / M_{W}$, not included in eq. (2.16), appear in 1PI vertex diagrams when external lepton masses are not neglected. We calculated such terms and proved explicitly that after performing the MI expansion they were suppressed by additional powers of $v^{2} / M_{\text {SUSY }}^{2}$ and therefore, a posteriori, negligible. Thus, we do not display such terms in eq. (2.16).
} 


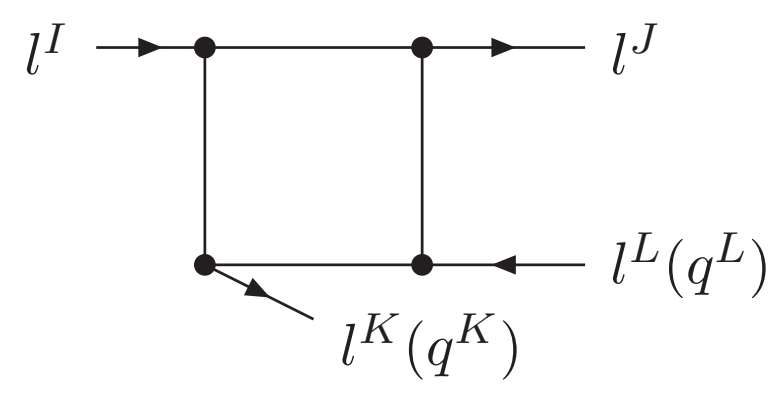

Figure 4. Box diagrams with external charged leptons or quarks.

$$
\begin{aligned}
& (4 \pi)^{2} \Gamma_{A}^{J I}\left(k_{J}, k_{I}\right)=-\sum_{n=1}^{4} \sum_{l, m=1}^{6} V_{\ell \tilde{L} N, L}^{J m n *} V_{\ell \tilde{L} N, L}^{I l n} V_{A \tilde{L} \tilde{L}}^{1 l m} m_{N_{n}} C_{0}\left[k_{J}, k_{I}-k_{J}, m_{N_{n}}, m_{\tilde{L}_{m}}, m_{\tilde{L}_{l}}\right] \\
& -\sum_{l, n=1}^{4} \sum_{m=1}^{6} V_{\ell \tilde{L} N, R}^{J n m *} V_{\ell \tilde{L} N, L}^{I n l}\left(V_{N A N, R}^{l 1 m} C_{2}+V_{N A N, L}^{l 1 m} m_{N_{l}} m_{N_{m}} C_{0}\right)\left[k_{J}, k_{I}-k_{J}, m_{\tilde{L}_{n}}, m_{N_{m}}, m_{N_{l}}\right],
\end{aligned}
$$

while the chargino-sneutrino triangle diagram is obtained by replacing $\tilde{L} \rightarrow \tilde{\nu}, N \rightarrow C$ and adjusting the summation limits appropriately in vertex factors $V_{\ldots}$ (see appendix A).

\subsection{Box contributions}

4 -fermion interactions are also generated by box diagrams. The corresponding conventions for incoming and outgoing particles are shown in figure 4 . We calculate all box diagrams in the approximation of vanishing external momenta. The effective Lagrangian for the 4-lepton interactions involves the quadrilinear operators

$$
\begin{aligned}
& O_{V X Y}^{J I K L}=\left(\bar{\ell}^{J} \gamma^{\mu} P_{X} \ell^{I}\right) \times\left(\bar{\ell}^{K} \gamma_{\mu} P_{Y} \ell^{L}\right), \\
& O_{S X Y}^{J I K L}=\left(\bar{\ell}^{J} P_{X} \ell^{I}\right) \times\left(\bar{\ell}^{K} P_{Y} \ell^{L}\right), \\
& O_{T X}^{J I K L}=\left(\bar{\ell}^{J} \sigma^{\mu \nu} \ell^{I}\right) \times\left(\bar{\ell}^{K} \sigma_{\mu \nu} P_{X} \ell^{L}\right),
\end{aligned}
$$

where $X, Y$ stands for the chirality $L$ or $R .^{5}$ The Wilson coefficients of these operators are calculated from the box diagrams in figure 4 and are denoted by $B_{N X Y}^{J I K L}$ with $N=V, S$, or $B_{T X}^{J I K L}$.

The operator basis in eq. (2.17) is redundant. First, we note that

$$
\begin{aligned}
& O_{N X Y}^{J I K L}=O_{N Y X}^{K L J I} \quad \text { for } N=V, S, \\
& O_{T X}^{J I K L}=O_{T X}^{K L J I} .
\end{aligned}
$$

Second, there are Fierz relations among different operators:

$$
\begin{aligned}
& O_{V X X}^{J I K L}=O_{V X X}^{K I J L}, \\
& O_{V X Y}^{J I K L}=-2 O_{S X Y}^{K I J L} \quad \text { for } X \neq Y,
\end{aligned}
$$

\footnotetext{
${ }^{5}$ Note that the upper $L$ index in box formfactors denotes the sfermion flavor while the lower $L$ subscript denotes its chirality, even if both symbols are identical. Also, recall that $\left(\bar{\ell}^{J} \sigma^{\mu \nu} P_{L} \ell^{I}\right) \times\left(\bar{\ell}^{K} \sigma_{\mu \nu} P_{R} \ell^{L}\right)=0$.
} 


$$
\begin{aligned}
& O_{T X}^{J I K L}=\frac{1}{2} O_{T X}^{K I J L}-6 O_{S X X}^{K I J L}, \\
& O_{S X X}^{J I K L}=-\frac{1}{2} O_{S X X}^{K I J L}-\frac{1}{8} O_{T X}^{K I J L} .
\end{aligned}
$$

Furthermore, we have

$$
\begin{array}{ll}
O_{V X Y}^{J I K L \dagger}=O_{V X Y}^{I J L K}, & O_{S L L}^{J I K L \dagger}=O_{S R R}^{I J L K}, \\
O_{S L R}^{J I K L \dagger}=O_{S R L}^{I J L K}, & O_{T L}^{J I K L \dagger}=O_{T R}^{J I L K} .
\end{array}
$$

Eqs. (2.18) to (2.20) must be taken into account when deriving the effective Lagrangian.

\subsubsection{Leptonic operators with $J \neq K$ and $I \neq L$}

The case with both $J \neq K$ and $I \neq L$ covers the decays $\tau^{\mp} \rightarrow \mu^{\mp} e^{\mp} \ell^{ \pm}$with $\ell=e$ or $\mu$, but does not appear in $\mu^{\mp}$ decays. We can therefore specify to $I=3$ for the effective Lagrangian. Furthermore, we can choose either $(J, K)=(1,2)$ or $(J, K)=(2,1)$ without the need to sum over both cases: the Fierz identities in eq. (2.19) permit to bring all operators into the form $(\bar{e} \ldots \tau) \times(\bar{\mu} \ldots \ell)$ (corresponding to the case $(J, K)=(1,2))$ or into an alternative form with $e$ interchanged with $\mu$. Thus we have

$$
L_{4 \ell}^{J 3 K L}=\sum_{L=1,2}\left[\sum_{\substack{N=V, S \\ X, Y=L, R}} B_{N X Y}^{J 3 K L} O_{N X Y}^{J 3 K L}+\sum_{X=L, R} B_{T X}^{J 3 K L} O_{T X}^{J 3 K L}\right]+\text { h.c. }
$$

with $J \neq K$ and $J, K, L \leq 2$,

as the four-lepton interaction in the Lagrangian. Note that the "+h.c." piece of $L_{4 \ell}^{J K}$ describes $\tau^{+}$decays.

The Wilson coefficients $B_{N X Y}^{J 3 K L}$ and $B_{T X}^{J 3 K L}$ in eq. (2.21) are simply identical to the results of the sum of all contributing box diagrams to the decay amplitude. The latter is given in eq. (3.7) with the coefficients of the spinor structure in the right column of table 1. The relation to the analytic expressions in eqs. (D.3) to (D.6) is

$$
B_{N X Y}^{J I K L}=B_{A N X Y}^{J I K L}+B_{B N X Y}^{J I K L}+B_{C N X Y}^{J I K L}+B_{D N X Y}^{J I K L}, \quad \text { for } N=V, S
$$

and an analogous expression for $B_{T X}^{J I K L}$.

\subsubsection{Leptonic operators with $J=K$ and $I \neq L$}

The case $J=K$ occurs for the decays $\mu^{ \pm} \rightarrow e^{ \pm} e^{ \pm} e^{\mp}$ and $\tau^{ \pm} \rightarrow \ell^{ \pm} \ell^{ \pm} \ell^{\mp \prime}$ with $\ell, \ell^{\prime}=e, \mu$. Thanks to the Fierz identities in eq. (2.19) we may restrict the operator basis to

$$
\begin{aligned}
O_{V X X}^{J I J L}, \quad O_{V X Y}^{J I J L}=-2 O_{S X Y}^{J I J L}, \quad O_{S X X}^{J I J L} & =-\frac{1}{12} O_{T X}^{J I J L}, \\
\text { with } X, Y & =L, R \text { and } X \neq Y .
\end{aligned}
$$


The four-lepton piece of the effective Lagrangian for the decay $\ell^{I \mp} \rightarrow \ell^{J \mp} \ell^{J \mp} \ell^{L \pm}$ reads:

$$
L_{4 \ell}^{J I J L}=\sum_{L=1,2}\left[\sum_{X, Y=L, R} \widetilde{C}_{V X Y}^{J I J L} O_{V X Y}^{J I J L}+\sum_{X=L, R} \widetilde{C}_{S X X}^{J I J L} O_{S X X}^{J I J L}\right]+\text { h.c. }
$$

with $L, J<I$.

For the matching calculation it is useful to quote the tree-level matrix elements of the operators:

$$
\begin{aligned}
\left\langle l^{J-}\left(p_{J}, s_{J}\right) l^{J-}\right. & \left.\left(p_{J}^{\prime}, s_{J}^{\prime}\right) l^{L+}\left(p_{L}, s_{J}\right)\left|O_{V X X}^{J J L}\right| l^{I-}\left(p_{I}, s_{I}\right)\right\rangle \\
= & {\left[\bar{u}\left(p_{J}, s_{J}\right) \gamma_{\mu} P_{X} u\left(p_{I}, s_{I}\right)\right]\left[\bar{u}\left(p_{J}^{\prime}, s_{J}^{\prime}\right) \gamma^{\mu} P_{X} v\left(p_{L}, s_{L}\right)\right] } \\
& -\left[\bar{u}\left(p_{J}^{\prime}, s_{J}^{\prime}\right) \gamma_{\mu} P_{X} u\left(p_{I}, s_{I}\right)\right]\left[\bar{u}\left(p_{J}, s_{J}\right) \gamma^{\mu} P_{X} v\left(p_{L}, s_{L}\right)\right] \\
= & 2\left[\bar{u}\left(p_{J}, s_{J}\right) \gamma_{\mu} P_{X} u\left(p_{I}, s_{I}\right)\right]\left[\bar{u}\left(p_{J}^{\prime}, s_{J}^{\prime}\right) \gamma^{\mu} P_{X} v\left(p_{L}, s_{L}\right)\right] \\
\left\langle l^{J-}\left(p_{J}, s_{J}\right) l^{J-}\right. & \left.\left(p_{J}^{\prime}, s_{J}^{\prime}\right) l^{L+}\left(p_{L}, s_{J}\right)\left|O_{V X Y}^{J I J}\right| l^{I-}\left(p_{I}, s_{I}\right)\right\rangle \\
= & {\left[\bar{u}\left(p_{J}, s_{J}\right) \gamma_{\mu} P_{X} u\left(p_{I}, s_{I}\right)\right]\left[\bar{u}\left(p_{J}^{\prime}, s_{J}^{\prime}\right) \gamma^{\mu} P_{Y} v\left(p_{L}, s_{L}\right)\right] } \\
& -\left[\bar{u}\left(p_{J}^{\prime}, s_{J}^{\prime}\right) \gamma_{\mu} P_{X} u\left(p_{I}, s_{I}\right)\right]\left[\bar{u}\left(p_{J}, s_{J}\right) \gamma^{\mu} P_{Y} v\left(p_{L}, s_{L}\right)\right] \\
= & {\left[\bar{u}\left(p_{J}, s_{J}\right) \gamma_{\mu} P_{X} u\left(p_{I}, s_{I}\right)\right]\left[\bar{u}\left(p_{J}^{\prime}, s_{J}^{\prime}\right) \gamma^{\mu} P_{Y} v\left(p_{L}, s_{L}\right)\right] } \\
& -2\left[\bar{u}\left(p_{J}, s_{J}\right) P_{X} u\left(p_{I}, s_{I}\right)\right]\left[\bar{u}\left(p_{J}^{\prime}, s_{J}^{\prime}\right) P_{Y} v\left(p_{L}, s_{L}\right)\right] \\
\left\langle l^{J-}\left(p_{J}, s_{J}\right) l^{J-}\right. & \left.\left(p_{J}^{\prime}, s_{J}^{\prime}\right) l^{L+}\left(p_{L}, s_{J}\right)\left|O_{S X X}^{J I J}\right| l^{I-}\left(p_{I}, s_{I}\right)\right\rangle \\
= & {\left[\bar{u}\left(p_{J}, s_{J}\right) P_{X} u\left(p_{I}, s_{I}\right)\right]\left[\bar{u}\left(p_{J}^{\prime}, s_{J}^{\prime}\right) P_{X} v\left(p_{L}, s_{L}\right)\right] } \\
& -\left[\bar{u}\left(p_{J}^{\prime}, s_{J}^{\prime}\right) P_{X} u\left(p_{I}, s_{I}\right)\right]\left[\bar{u}\left(p_{J}, s_{J}\right) P_{X} v\left(p_{L}, s_{L}\right)\right] \\
= & \frac{1}{2}\left[\bar{u}\left(p_{J}, s_{J}\right) P_{X} u\left(p_{I}, s_{I}\right)\right]\left[\bar{u}\left(p_{J}^{\prime}, s_{J}^{\prime}\right) P_{X} v\left(p_{L}, s_{L}\right)\right] \\
& -\frac{1}{8}\left[\bar{u}\left(p_{J}, s_{J}\right) \sigma_{\mu \nu} P_{X} u\left(p_{I}, s_{I}\right)\right]\left[\bar{u}\left(p_{J}^{\prime}, s_{J}^{\prime}\right) \sigma^{\mu \nu} P_{X} v\left(p_{L}, s_{L}\right)\right]
\end{aligned}
$$

Here we have used the Fierz transform to group the spinors into the canonical order $\left[\bar{u}\left(p_{J}, \ldots\right) \ldots u\left(p_{I}, \ldots\right)\right]\left[\bar{u}\left(p_{J}^{\prime}, \ldots\right) \ldots v\left(p_{L}, \ldots\right)\right]$. This allows us to use the same formula for spin-summed squared matrix elements as in the case of $J \neq K$ of section 2.4.1.

To quote the Wilson coefficients $\widetilde{C}_{N X Y}^{J I J L}, N=V, S$ in terms of the box diagrams $B_{N X Y}^{J I J L}$ in eq. (2.22) we must compare the results of the MSSM decay amplitude in eq. (3.6) with the matrix elements in eq. (2.25) and read off coefficients of the various Dirac structures. The result is

$$
\begin{aligned}
\widetilde{C}_{V X X}^{J I J L} & =\frac{1}{2} B_{V X X}^{J I J L}, \\
\widetilde{C}_{V X Y}^{J I J L} & =B_{V X Y}^{J I J L} \\
\widetilde{C}_{S X X}^{J I J L} & =2 B_{S X X}^{J I J L} .
\end{aligned} \quad \text { for } X \neq Y,
$$

The Fierz identities further imply the equalities

$$
\begin{aligned}
& B_{S X Y}^{J I J L}=-2 B_{V X Y}^{J I J L} \quad \text { for } X \neq Y, \\
& B_{T X}^{J I J L}=-\frac{1}{4} B_{S X X}^{J I J L} .
\end{aligned}
$$




\subsubsection{Leptonic operators with $J=K$ and $I=L$}

These operators do not appear in lepton decays, but trigger muonium-antimuonium transitions and describe muon or tau pair production in $e^{-}-e^{-}$collisions at energies far below $M_{\text {SUSY }}$. Their Wilson coefficients are tiny in the MSSM.

\subsubsection{Operators with two leptons and two quarks}

The analogous Lagrangian for the 2-lepton-2-quark interactions reads

$$
L_{2 \ell 2 q}^{I J K L}=\sum_{N, X, Y} B_{q N X Y}^{I J K L} O_{q N X Y}^{J I K L}
$$

where

$$
\begin{aligned}
O_{q V X Y}^{I J K L} & =\left(\bar{\ell}_{I} \gamma^{\mu} P_{X} \ell_{J}\right) \times\left(\bar{q}_{K} \gamma_{\mu} P_{Y} q_{L}\right), \\
O_{q S X Y}^{I J K L} & =\left(\bar{\ell}_{I} P_{X} \ell_{J}\right) \times\left(\bar{q}_{L} P_{Y} q_{K}\right), \\
O_{q T X}^{I J K L} & =\left(\bar{\ell}_{I} \sigma^{\mu \nu} \ell_{J}\right) \times\left(\bar{q}_{K} \sigma_{\mu \nu} P_{X} q_{L}\right) .
\end{aligned}
$$

Again, we consider only purely leptonic contributions here in detail and do not give explicit expressions for the 2-lepton-2-quark box diagrams. The relevant expressions in the mass eigenbasis can be found using formulae of appendix $\mathrm{D}$ and inserting proper quark vertices from refs. [43, 44] into these.

\section{Observables}

In this section we collect the formulae for the LFV observables in terms of the effective interactions defined in section 2. All the processes listed here will be included in the future version of the SUSY_FLAVOR numerical library calculating an extensive set of flavour and CP-violating observables both in the quark and leptonic sectors [66-68].

\subsection{Radiative lepton decays: $\ell^{I} \rightarrow \ell^{J} \gamma$}

The branching ratios for the radiative lepton decays $\ell^{I} \rightarrow \ell^{J} \gamma$ are given by

$$
\operatorname{Br}\left(\ell^{I} \rightarrow \ell^{J} \gamma\right)=\frac{48 \pi^{2} e^{2}}{m_{I}^{2} G_{F}^{2}}\left(\left|F_{\gamma}^{J I}\right|^{2}+\left|F_{\gamma}^{I J}\right|^{2}\right) \operatorname{Br}\left(\ell^{I} \rightarrow e \nu \nu\right)
$$

Here we used $\Gamma\left(\ell^{I} \rightarrow e \nu \nu\right) \approx G_{F}^{2} m_{I}^{5} /\left(192 \pi^{3}\right)$ for the tree-level leptonic decay width and the factors $\operatorname{Br}(\mu \rightarrow e \nu \nu) \approx 1, \operatorname{Br}(\tau \rightarrow e \nu \nu)=0.1785 \pm 0.0005$ [69] are introduced to account for the hadronic decay modes of the $\tau$ lepton.

Even though in our numerical analyses we restrict ourselves to LFV processes, we remind the reader that the expressions for the anomalous magnetic moments and electric dipole moments of the charged leptons can be also calculated in term of the quantities defined in eq. (2.4) and read:

$$
\begin{aligned}
\Delta a_{I} & =-4 m_{I} \operatorname{Re}\left[F_{\gamma A}^{I I}-m_{I}\left(F_{\gamma L B}^{I I}+F_{\gamma R B}^{I I}\right)\right], \\
d_{l}^{I} & =-2 e \operatorname{Im} F_{\gamma A}^{I I}
\end{aligned}
$$


I)

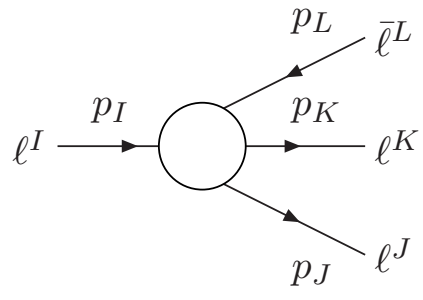

II)

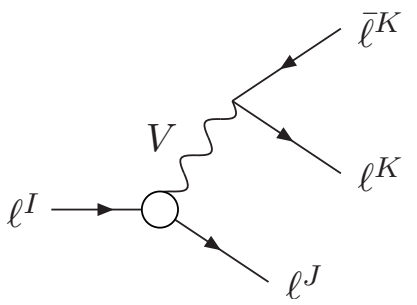

Figure 5. Diagrams contributing to $\ell^{I} \rightarrow \ell^{J} \ell^{K} \bar{\ell}^{L}$ decay. I): 1PI irreducible box diagrams; II): penguin diagrams with $V=Z, \gamma, h, H$ or $A$. For $K=J$ crossed diagrams must be also included.

\section{$3.2 \quad h(H) \rightarrow \bar{\ell}^{I} \ell^{J}$ decays}

The decay branching ratios for the CP-even and CP-odd Higgs bosons read:

$$
\begin{aligned}
\operatorname{Br}\left(H_{0}^{K} \rightarrow \ell^{I+} \ell^{J-}\right) & =\frac{m_{H_{0}^{K}}}{16 \pi \Gamma_{H_{0}^{K}}}\left(\left|F_{h}^{I J K}\right|^{2}+\left|F_{h}^{J I K}\right|^{2}\right) \\
\operatorname{Br}\left(A_{0} \rightarrow \ell^{I+} \ell^{J-}\right) & =\frac{m_{A}}{16 \pi \Gamma_{A}}\left(\left|F_{A}^{I J}\right|^{2}+\left|F_{A}^{J I}\right|^{2}\right)
\end{aligned}
$$

with $F_{h}^{I J K}, F_{A}^{I J}$ defined in eq. (2.14). Note that summing over lepton charges in the final state, $\ell^{I+} \ell^{J-}$ and $\ell^{J+} \ell^{I-}$, would produce an additional factor of 2 .

\section{$3.3 \quad \ell^{I} \rightarrow \ell^{J} \ell^{K} \bar{\ell}^{L}$ decays}

The LFV decays of charged lepton into three lighter ones can be divided into 3 classes, depending on the flavours in the final state:

(A) $\ell \rightarrow \ell^{\prime} \ell^{\prime} \ell^{\prime}$ : three leptons of the same flavour, i.e. $\mu^{ \pm} \rightarrow e^{ \pm} e^{+} e^{-}, \tau^{ \pm} \rightarrow e^{ \pm} e^{+} e^{-}$and $\tau^{ \pm} \rightarrow \mu^{ \pm} \mu^{+} \mu^{-}$, with a pair of opposite charged leptons.

(B) $\ell^{ \pm} \rightarrow \ell^{\prime \pm} \ell^{\prime \prime+} \ell^{\prime \prime-}$ : three distinguishable leptons with $\ell^{\prime}$ carrying the same charge as $\ell$, i.e. $\tau^{ \pm} \rightarrow e^{ \pm} \mu^{+} \mu^{-}$and $\tau^{ \pm} \rightarrow \mu^{ \pm} e^{+} e^{-}$.

(C) $\ell^{ \pm} \rightarrow \ell^{\prime \mp} \ell^{\prime \prime+} \ell^{\prime \prime-}$ : three distinguishable leptons with $\ell^{\prime}$ carrying the opposite charge as $\ell$, i.e. $\tau^{ \pm} \rightarrow e^{\mp} \mu^{ \pm} \mu^{ \pm}$and $\tau^{ \pm} \rightarrow \mu^{\mp} e^{ \pm} e^{ \pm}$.

Class (C), representing a $\Delta L=2$ processes, is tiny within the MSSM: it could only be generated at 1-loop level by box diagrams suppressed by double flavour changes, or at the 2-loop level by double penguin diagrams involving two LFV vertices. Therefore, we will not consider these processes in our numerical analysis.

In order to calculate $\operatorname{Br}\left(\ell^{I} \rightarrow \ell^{J} \ell^{K} \bar{\ell}^{L}\right)$ we decompose the corresponding amplitude $A$ as

$$
A=A_{0}+A_{\gamma} .
$$

The relevant diagrams are displayed in figure 5. $A_{0}$ contains contributions from 4-lepton box diagrams and from penguin diagrams (including vector-like off-shell photon couplings, see eq. (2.5)) which in the limit of vanishing external momenta can be represented as the 
4 -fermion contact interactions. $A_{\gamma}$ is the on-shell photon contribution originating from the magnetic operator (see eq. (2.2)) which has to be treated separately with more care as the photon propagator becomes singular in the limit of vanishing external momenta.

We further decompose $A_{0}$ for the two cases (A) and (B) according to its Lorentz structure:

$$
\begin{aligned}
& A_{0}^{(A)}=\sum_{Q=V, S, T} C_{Q, X Y}^{(A)}\left[\bar{u}\left(p_{J}\right) \Gamma_{Q}^{\prime} P_{X} u\left(p_{I}\right)\right]\left[\bar{u}\left(p_{J}^{\prime}\right) \Gamma_{Q} P_{Y} v\left(p_{L}\right)\right], \\
& A_{0}^{(B)}=\sum_{Q=V, S, T} C_{Q, X Y}^{(B)}\left[\bar{u}\left(p_{J}\right) \Gamma_{Q}^{\prime} P_{X} u\left(p_{I}\right)\right]\left[\bar{u}\left(p_{K}\right) \Gamma_{Q} P_{Y} v\left(p_{L}\right)\right],
\end{aligned}
$$

with $X, Y=L, R$. Note that the amplitude $A_{0}^{(A)}$ in general contains a second term which is obtained from the one given in eq. (3.6) by replacing $\left(p_{J} \leftrightarrow p_{J}^{\prime}\right)$. However, one can use Fierz identities to reduce it to the structure given in eq. (3.6). The basis of Dirac quadrilinears $\Gamma_{Q}$ is the same as the one used to decompose 4-lepton box diagrams in eq. (2.17):

$$
\Gamma_{S}=1, \quad \Gamma_{V}=\gamma^{\mu}, \quad \Gamma_{T}=\sigma_{\mu \nu},
$$

and $\Gamma_{Q}^{\prime}$ is obtained from $\Gamma_{Q}$ by lowering the Lorentz indices.

The amplitudes originating from on-shell photon exchange are given by

$$
\begin{aligned}
A_{\gamma}^{(A)}= & \frac{e}{\left(p_{I}-p_{J}\right)^{2}}\left[\bar{u}\left(p_{J}\right) i \sigma^{\mu \nu}\left(C_{\gamma L} P_{L}+C_{\gamma R} P_{R}\right)\left(p_{I}-p_{J}\right)_{\nu} u\left(p_{I}\right)\right]\left[\bar{u}\left(p_{J}^{\prime}\right) \gamma_{\mu} v\left(p_{L}\right)\right] \\
& -\left(p_{J} \leftrightarrow p_{J}^{\prime}\right) \\
A_{\gamma}^{(B)}= & \frac{e}{\left(p_{I}-p_{J}\right)^{2}}\left[\bar{u}\left(p_{J}\right) i \sigma^{\mu \nu}\left(C_{\gamma L} P_{L}+C_{\gamma R} P_{R}\right)\left(p_{I}-p_{J}\right)_{\nu} u\left(p_{I}\right)\right]\left[\bar{u}\left(p_{K}\right) \gamma_{\mu} v\left(p_{L}\right)\right] .
\end{aligned}
$$

The full form of the coefficients $C_{N}^{(A, B)}, C_{\gamma}$ is displayed in table 1, where we compactified the expressions by using the following abbreviations for the Higgs penguin contributions: ${ }^{6}$

$$
V_{H}^{J I}=\sum_{N=1}^{2} \frac{Z_{R}^{1 N}}{m_{H_{0}^{N}}^{2}} F_{h}^{J I N}, \quad V_{A}^{J I}=\frac{i \sin \beta}{m_{A_{0}}^{2}} F_{A}^{J I} .
$$

Note that in eq. (3.7) and eq. (3.9) we do not explicitly display flavour indices, but they are specified in table 1.

Neglecting the lighter lepton masses whenever possible, the expression for the branching ratios can be written down as (for comparison see [23]):

$$
\begin{aligned}
\operatorname{Br}\left(\ell^{I} \rightarrow \ell^{J} \ell^{K} \bar{\ell}^{L}\right)= & \frac{N_{c} \operatorname{Br}\left(\ell^{I} \rightarrow e \nu \nu\right)}{32 G_{F}^{2}}\left(4\left(\left|C_{V L L}\right|^{2}+\left|C_{V R R}\right|^{2}+\left|C_{V L R}\right|^{2}+\left|C_{V R L}\right|^{2}\right)\right. \\
& +\left|C_{S L L}\right|^{2}+\left|C_{S R R}\right|^{2}+\left|C_{S L R}\right|^{2}+\left|C_{S R L}\right|^{2} \\
& \left.+48\left(\left|C_{T L}\right|^{2}+\left|C_{T R}\right|^{2}\right)+X_{\gamma}\right)
\end{aligned}
$$

where $N_{c}=1 / 2$ if two of the final state leptons are identical (decays (A)), $N_{c}=1$ for decays (B) and $X_{\gamma}$ denotes the contribution to matrix element from the photon penguin $A_{\gamma}$,

\footnotetext{
${ }^{6}$ Note that we define lepton Yukawa coupling appearing in table 1 to be negative, $Y_{l}^{I}=-\sqrt{2} m_{l}^{I} / v_{1}$.
} 


\begin{tabular}{|l|l|l|}
\hline & Decay (A) & Decay (B) \\
\hline$C_{V L L}$ & $B_{V L L}^{J I J J}-\frac{e\left(1-2 s_{W}^{2}\right)}{s_{W} c_{W}^{2}} F_{Z L}^{J I}+2 e^{2} V_{\gamma L}^{J I}$ & $B_{V L L}^{J I K K}-\frac{e\left(1-2 s_{W}^{2}\right)}{2 s_{W} c_{W} M_{Z}^{2}} F_{Z L}^{J I}+e^{2} V_{\gamma L}^{J I}$ \\
$C_{V R R}$ & $B_{V R R}^{J I J J}+\frac{2 e s_{W}}{c_{W} M_{Z}^{2}} F_{Z R}^{J I}+2 e^{2} V_{\gamma R}^{J I}$ & $B_{V R R}^{J I K K}+\frac{e s_{W}}{c_{W} M_{Z}^{2}} F_{Z R}^{J I}+e^{2} V_{\gamma R}^{J I}$ \\
$C_{V L R}$ & $B_{V L R}^{J I J J}+\frac{e s_{W}}{c_{W} M_{Z}^{2}} F_{Z L}^{J I}+e^{2} V_{\gamma L}^{J I}+\frac{1}{2} Y_{l}^{J}\left(V_{H}^{I J *}-V_{A}^{I J *}\right)$ & $B_{V L R}^{J I K K}+\frac{e s_{W}}{c_{W} M_{Z}^{2}} F_{Z L}^{J I}+e^{2} V_{\gamma L}^{J I}$ \\
$C_{V R L}$ & $B_{V R L}^{J I J J}-\frac{e\left(1-2 s_{W}^{2}\right)}{2 s_{W} c_{W} M_{Z}^{2}} F_{Z R}^{J I}+e^{2} V_{\gamma R}^{J I}+\frac{1}{2} Y_{l}^{J}\left(V_{H}^{J I}-V_{A}^{J I}\right)$ & $B_{V R L}^{J I K K}-\frac{e\left(1-2 s_{W}^{2}\right)}{2 s_{W} c_{W} M_{Z}^{2}} F_{Z R}^{J I}+e^{2} V_{\gamma R}^{J I}$ \\
$C_{S L L}$ & $B_{S L L}^{J I J J}+\frac{3}{2} Y_{l}^{J}\left(V_{H}^{J I}+V_{A}^{J I}\right)$ & $B_{S L L}^{J I K K}+Y_{l}^{K}\left(V_{H}^{J I}+V_{A}^{J I}\right)$ \\
$C_{S R R}$ & $B_{S R R}^{J I J J}+\frac{3}{2} Y_{l}^{J}\left(V_{H}^{I J *}+V_{A}^{I J *}\right)$ & $B_{V R R}^{J I K K}+Y_{l}^{K}\left(V_{H}^{I J *}+V_{A}^{I J *}\right)$ \\
$C_{S L R}$ & $-2 B_{V L R}^{J I J J}-\frac{2 e s_{W}}{c_{W} M_{Z}^{2}} F_{Z L}^{J I}-2 e^{2} V_{\gamma L}^{J I}+Y_{l}^{J}\left(V_{H}^{J I}-V_{A}^{J I}\right)$ & $B_{S L R}^{J I K K}+Y_{l}^{K}\left(V_{H}^{J I}-V_{A}^{J I}\right)$ \\
$C_{S R L}$ & $-2 B_{S R L}^{J I J J}+\frac{e\left(1-2 s_{W}^{2}\right)}{s_{W} c_{W} M_{Z}^{2}} F_{Z R}^{J I}-2 e^{2} V_{\gamma R}^{J I}+Y_{l}^{J}\left(V_{H}^{I J *}-V_{A}^{I J *}\right)$ & $B_{S R L}^{J I K K}+Y_{l}^{K}\left(V_{H}^{I J *}-V_{A}^{I J *}\right)$ \\
$C_{T L}$ & $-\frac{1}{4} B_{S L L}^{J I J J}+\frac{1}{8} Y_{l}^{J}\left(V_{H}^{J I}+V_{A}^{J I}\right)$ & $B_{T L}^{J I K K}$ \\
$C_{T R}$ & $-\frac{1}{4} B_{S R R}^{J I J J}+\frac{1}{8} Y_{l}^{J}\left(V_{H}^{I J *}+V_{A}^{I J *}\right)$ & $B_{T R}^{J I K K}$ \\
$C_{\gamma L}$ & $-2 e F_{\gamma}^{J I}$ & $-2 e F_{\gamma}^{J I}$ \\
$C_{\gamma R}$ & $-2 e F_{\gamma}^{I J *}$ & $-2 e F_{\gamma}^{I J *}$ \\
\hline
\end{tabular}

Table 1. Coefficients $C_{N}, C_{\gamma}$ of eq. (3.7) and eq. (3.9) for decay types (A) and (B). $B_{Q X Y}, B_{T X}$ denote the irreducible box diagram contributions (see eq. (2.21)), the terms with $F_{Z}$ stem from the $Z$ penguin Lagrangian (eq. (2.7)), $V_{\gamma}$ is the sum of the vector-like photon contributions (eq. (2.5)), Higgs contributions are defined in eq. (3.10) and the coefficients $F_{\gamma}$ of the magnetic operator are defined in eq. (2.2).

including also its interference with the $A_{0}$ part of the amplitude ( $m$ denotes the mass of the heaviest final state lepton)

$$
\begin{aligned}
X_{\gamma}^{(A)}= & -\frac{16 e}{m_{\ell^{I}}} \operatorname{Re}\left[\left(2 C_{V L L}+C_{V L R}-\frac{1}{2} C_{S L R}\right) C_{\gamma R}^{\star}+\left(2 C_{V R R}+C_{V R L}-\frac{1}{2} C_{S R L}\right) C_{\gamma L}^{\star}\right] \\
& +\frac{64 e^{2}}{m_{\ell^{I}}^{2}}\left(\log \frac{m_{\ell^{I}}^{2}}{m^{2}}-\frac{11}{4}\right)\left(\left|C_{\gamma L}\right|^{2}+\left|C_{\gamma R}\right|^{2}\right) \\
X_{\gamma}^{(B)}= & -\frac{16 e}{m_{\ell^{I}}} \operatorname{Re}\left[\left(C_{V L L}+C_{V L R}\right) C_{\gamma R}^{\star}+\left(C_{V R R}+C_{V R L}\right) C_{\gamma L}^{\star}\right] \\
& +\frac{32 e^{2}}{m_{\ell^{I}}^{2}}\left(\log \frac{m_{\ell^{I}}^{2}}{m^{2}}-3\right)\left(\left|C_{\gamma L}\right|^{2}+\left|C_{\gamma R}\right|^{2}\right) .
\end{aligned}
$$

\section{$3.4 \mu \rightarrow e$ conversion in nuclei}

The full 1-loop expressions for the $\mu \rightarrow e$ conversion in Nuclei depend on both the squark and slepton SUSY breaking terms. Thus, in principle the resulting upper bounds on the slepton mass insertions to some extent depend on the squark masses. Therefore, we do not include $\mu \rightarrow e$ conversion in nuclei in our numerical analysis. ${ }^{7}$ However, for completeness we collect here the complete set of formulae required to calculate the rate of this process.

\footnotetext{
${ }^{7}$ Recent discussion of interplay between the bounds on MI's in the slepton and squark sectors can be found in ref. [70].
} 
$\mu \rightarrow e$ conversion in nuclei is produced by the dipole, the vector, and the scalar operators already at the tree level [71]. Following the discussion of ref. [72] we use the effective Lagrangian

$$
L_{\mu \rightarrow e}=\sum_{N, X, Y} C_{q_{I} q_{I}}^{N X Y} O_{N X Y}^{q_{I} q_{I}}+C_{X}^{g g} O_{X}^{g g}
$$

where $N=V, S$ and $X, Y=L, R$ with the operators defined as

$$
\begin{aligned}
O_{V X Y}^{q_{I} q_{I}} & =\left(\bar{e} \gamma^{\mu} P_{X} \mu\right)\left(\bar{q}_{I} \gamma_{\mu} P_{Y} q_{I}\right) \\
O_{S X Y}^{q_{I} q_{I}} & =\left(\bar{e} P_{X} \mu\right)\left(\bar{q}_{I} P_{Y} q_{I}\right) \\
O_{X}^{g g} & =\alpha_{s} m_{\mu} G_{F}\left(\bar{e} P_{X} \mu\right) G_{\mu \nu}^{a} G_{a}^{\mu \nu}
\end{aligned}
$$

Using the notation introduced in previous sections, the corresponding Wilson coefficients can be expressed as

$$
\begin{aligned}
& C_{V X L}^{d_{I} d_{I}}=C_{d \ell V X L}^{12 I I}-\frac{1}{m_{Z}^{2}} \frac{e}{2 s_{W} c_{W}}\left(1-\frac{2}{3} s_{W}^{2}\right) F_{Z X}^{12}-\frac{1}{3} e^{2} V_{\gamma X}^{J I} \\
& C_{V X R}^{d_{I} d_{I}}=C_{d \ell V X R}^{12 I I}+\frac{1}{m_{Z}^{2}} \frac{e}{3 s_{W} c_{W}} s_{W}^{2} F_{Z X}^{12}-\frac{1}{3} e^{2} V_{\gamma X}^{J I} \\
& C_{V X L}^{u_{I} u_{I}}=C_{u \ell V X L}^{12 I I}+\frac{1}{m_{Z}^{2}} \frac{e}{2 s_{W} c_{W}}\left(1-\frac{4}{3} s_{W}^{2}\right) F_{Z X}^{12}+\frac{2}{3} e^{2} V_{\gamma X}^{J I} \\
& C_{V X R}^{u_{I} u_{I}}=C_{u \ell V X R}^{12 I I}-\frac{1}{m_{Z}^{2}} \frac{e}{s_{W} c_{W}} \frac{2}{3} s_{W}^{2} F_{Z X}^{12}+\frac{2}{3} e^{2} V_{\gamma X}^{J I} \\
& C_{S L X}^{d_{I} d_{I}}=C_{d \ell S L X}^{12 I I}+\frac{1}{\left(m_{0}^{K}\right)^{2}} F_{h}^{12 K} F_{h d}^{I I K} \\
& C_{S L X}^{u_{I} u_{I}}=C_{u \ell S L X}^{12 I I}+\frac{1}{\left(m_{0}^{K}\right)^{2}} F_{h}^{12 K} F_{h u}^{I I K} \\
& C_{S R X}^{d_{I} d_{I}}=C_{d \ell S R X}^{12 I I}+\frac{1}{\left(m_{0}^{K}\right)^{2}} F_{h}^{21 K *} F_{h d}^{I I K} \\
& C_{S R X}^{u_{I} u_{I}}=C_{u \ell S R X}^{12 I I}+\frac{1}{\left(m_{0}^{K}\right)^{2}} F_{h}^{21 K *} F_{h u}^{I I K}
\end{aligned}
$$

For this process, a Lagrangian involving only quark, lepton and photon fields is not sufficient. Instead, an effective Lagrangian at the nucleon level containing proton and neutron fields is required. It can be obtained in two steps. First, heavy quarks are integrated out. This results in a redefinition of the Wilson coefficient of the gluonic operator [73]

$$
C_{L}^{g g} \rightarrow \tilde{C}_{L}^{g g}=C_{L}^{g g}-\frac{1}{12 \pi} \sum_{q=c, b} \frac{C_{S L L}^{q q}+C_{S L R}^{q q}}{G_{F} m_{\mu} m_{q}}
$$

with an analogous equation for $C_{g g}^{R}$. Second, the resulting Lagrangian is matched at the scale of $\mu_{n}=1 \mathrm{GeV}$ to an effective Lagrangian at the nucleon level. Following [74] the transition rate $\Gamma_{\mu \rightarrow e}^{N}=\Gamma\left(\mu^{-} N \rightarrow e^{-} N\right)$ can then be written as

$$
\begin{aligned}
\Gamma_{\mu \rightarrow e}^{N}= & \frac{m_{\mu}^{5}}{4}\left|-e C_{L}^{D} F_{\gamma}^{12} / m_{\mu}+4\left(G_{F} m_{\mu} m_{p} \tilde{C}_{S L}^{(p)} S_{N}^{(p)}+\tilde{C}_{V R}^{(p)} V_{N}^{(p)}+(p \rightarrow n)\right)\right|^{2} \\
& +(L \leftrightarrow R),
\end{aligned}
$$


where $p$ and $n$ denote the proton and the neutron, respectively. The effective couplings in eq. (3.17) can be expressed in terms of our Wilson coefficients as

$$
\begin{aligned}
& \tilde{C}_{V R}^{(p / n)}=\sum_{q=u, d, s}\left(C_{V R L}^{q q}+C_{V R R}^{q q}\right) f_{V p / n}^{(q)} \\
& \tilde{C}_{S L}^{(p / n)}=\sum_{q=u, d, s} \frac{\left(C_{S L L}^{q q}+C_{S L R}^{q q}\right)}{m_{\mu} m_{q} G_{F}} f_{S p / n}^{(q)}+\tilde{C}_{L}^{g g} f_{G p / n}
\end{aligned}
$$

with analogous relations for $L \leftrightarrow R$. The Wilson coefficients in eqs. (3.18) and (3.19) are to be evaluated at the scale $\mu_{n}$.

The nucleon form factors for vector operators are fixed by vector-current conservation, i.e. $f_{V p}^{(u)}=2, f_{V n}^{(u)}=1, f_{V p}^{(d)}=1, f_{V n}^{(d)}=2, f_{V p}^{(s)}=0, f_{V n}^{(s)}=0$. Hence, the sum in eq. (3.18) is in fact only over $q=u, d$. The calculation of the scalar form factors are more involving. The values of the up- and down-quark scalar couplings $f_{S p / n}^{(u / d)}$ (based on the two-flavour chiral perturbation theory framework of [75]) can be found in refs. [76, 77], while the values of the $s$-quark scalar couplings $f_{S p / n}^{(s)}$ can be borrowed from a lattice calculation [78]. ${ }^{8}$ In summary, one has

$$
\begin{array}{ll}
f_{S p}^{(u)}=(20.8 \pm 1.5) \times 10^{-3}, & f_{S n}^{(u)}=(18.9 \pm 1.4) \times 10^{-3}, \\
f_{S p}^{(d)}=(41.1 \pm 2.8) \times 10^{-3}, & f_{S n}^{(d)}=(45.1 \pm 2.7) \times 10^{-3}, \\
f_{S p}^{(s)}=f_{S n}^{(s)}=(53 \pm 27) \times 10^{-3} . &
\end{array}
$$

The form factor for the gluonic operator can be obtained from a sum rule. In our normalisation

$$
f_{G p / n}=-\frac{8 \pi}{9}\left(1-\sum_{q=u, d, s} f_{S p / n}^{(q)}\right)
$$

The quantities $D_{N}, S_{N}^{(p / n)}$, and $V_{N}^{(p / n)}$ in eq. (3.17) are related to the overlap integrals [81] between the lepton wave functions and the nucleon densities. They depend on the nature of the target $N$. Their numerical values can be found in ref. [71]:

$$
\begin{aligned}
& D_{\mathrm{Au}}=0.189, \quad S_{\mathrm{Au}}^{(p)}=0.0614, \quad V_{\mathrm{Au}}^{(p)}=0.0974, \quad S_{\mathrm{Au}}^{(n)}=0.0918, \quad V_{\mathrm{Au}}^{(n)}=0.146 ; \\
& D_{\mathrm{Al}}=0.0362, \quad S_{\mathrm{Al}}^{(p)}=0.0155, \quad V_{\mathrm{Al}}^{(p)}=0.0161, \quad S_{\mathrm{Al}}^{(n)}=0.0167, \quad V_{\mathrm{Al}}^{(n)}=0.0173 ;
\end{aligned}
$$

for gold and aluminium, respectively.

Finally, the branching ratio is defined as the transition rate, (see eq. (3.17)), divided by the capture rate, the latter given in ref. [82]:

$$
\Gamma_{\mathrm{Au}}^{\mathrm{capt}}=8.7 \times 10^{-15} \mathrm{MeV}, \quad \Gamma_{\mathrm{Al}}^{\mathrm{capt}}=4.6 \times 10^{-16} \mathrm{MeV} .
$$

\footnotetext{
${ }^{8}$ For earlier determinations of the pion-nucleon sigma terms see [79, 80].
} 


\section{Mass eigenstates vs. mass insertions calculations}

For each process, we have given the exact one-loop expressions calculated in the mass eigenbasis (ME). These formulae are compact and well suited for numerical computations, however, do not allow for an easy understanding of the qualitative behaviour of the LFV amplitudes for various choices of the MSSM parameters. Therefore, in this section we expand the Wilson coefficients in terms of the "mass insertions", defined as the off-diagonal elements (both flavour violating and flavour conserving) of the mass matrices. Such an expansion allows us to:

- Recover the direct analytical dependence of the results on the MSSM Lagrangian parameters.

- Prove analytically the expected decoupling features of the amplitudes in the limit of a heavy SUSY spectrum. In the case of Higgs boson decays, we also identify explicitly the terms decoupling only with the heavy CP-odd Higgs mass $M_{A}$ (which also determines the heavy $\mathrm{CP}$ even and the charged Higgs masses). The decoupling properties also serve as an important cross-check of the correctness of our calculations.

- Test the dependence of the results on the pattern of the MSSM spectrum and the size of the mass splitting between SUSY particles.

- Better understand the possible cancellations between various types of contributions and correlations between different LFV processes.

The mass insertion expansion in flavour off-diagonal terms has been used for a long time in numerous articles on the subject. However, often various simplifying assumptions have been made, i.e. some terms have been neglected or a simplified pattern of the slepton spectrum was considered. This is understandable as a consistent MI expansion of the amplitudes for the LFV processes in the MSSM, mediated by the virtual chargino and neutralino exchanges, is technically challenging. The standard approach used in literature is to calculate diagrammatically the LFV amplitudes with the "mass insertions" treated as the new interaction vertices. We follow the common practice and normalise such slepton mass insertions to dimensionless " $\Delta$-parameters" : 9

$$
\begin{aligned}
\Delta_{L L}^{I J} & =\frac{\left(M_{L L}^{2}\right)^{I J}}{\sqrt{\left(M_{L L}^{2}\right)^{I I}\left(M_{L L}^{2}\right)^{J J}}}, & \Delta_{R R}^{I J} & =\frac{\left(M_{R R}^{2}\right)^{I J}}{\sqrt{\left(M_{R R}^{2}\right)^{I I}\left(M_{R R}^{2}\right)^{J J}}}, \\
\Delta_{L R}^{I J} & =\frac{A_{l}^{I J}}{\left(\left(M_{L L}^{2}\right)^{I I}\left(M_{R R}^{2}\right)^{J J}\right)^{1 / 4}}, & \Delta_{L R}^{\prime I J} & =\frac{A_{l}^{\prime I J}}{\left(\left(M_{L L}^{2}\right)^{I I}\left(M_{R R}^{2}\right)^{J J}\right)^{1 / 4}},
\end{aligned}
$$

where $M_{L L}^{2}, M_{R R}^{2}, A_{l}, A_{l}^{\prime}$ are the slepton soft mass matrices and trilinear terms.

As lepton flavour violation is already strongly constrained experimentally, it is sufficient to expand the amplitudes up to the first order in flavour-violating $\Delta$ 's. For instance, the

\footnotetext{
${ }^{9}$ We assume that trilinear $A_{l}, A_{l}^{\prime}$ terms scale linearly with the slepton mass scale.
} 
effective vertices listed in section 3 take the schematic form:

$$
\begin{aligned}
F^{I J}=\frac{1}{(4 \pi)^{2}}( & F_{L L}^{I J} \Delta_{L L}^{I J}+F_{R R}^{I J} \Delta_{R R}^{J I} \\
& \left.\quad+F_{A L R}^{I J} \Delta_{L R}^{J I}+F_{B L R}^{I J} \Delta_{L R}^{I J *}+F_{A L R}^{\prime I J} \Delta_{L R}^{\prime J I}+F_{B L R}^{\prime I J} \Delta_{L R}^{\prime I J *}\right)
\end{aligned}
$$

The MSSM contributions to $F_{L L}, \ldots, F_{B L R}^{\prime}$ can be classified according to their decoupling behaviour, distinguishing the following types ( $M$ denotes the average SUSY mass scale):

1. Effects related to the diagonal trilinear slepton soft terms or to the off-diagonal elements of supersymmetric fermion mass matrices, decoupling as $v^{2} / M^{2}$.

2. Effects related to the external momenta of the (on-shell) Higgs or $Z^{0}$ bosons, decoupling as $M_{h}^{2} / M^{2}$ or $M_{Z}^{2} / M^{2}$ (we did not include the $M_{Z}$ dependence as it is not necessary for the considered processes).

3. Non-decoupling effects related to the 2HDM structure of the MSSM. Such contributions are constant in the limit of a heavy SUSY scale $M$ but, in case of the SM-like Higgs boson $h$, decouple with the CP-odd Higgs mass like $v^{2} / M_{A}^{2}$ (the effective couplings of heavier $H, A$ bosons do not exhibit such a suppression). They are proportional either to the lepton Yukawa couplings or to the non-holomorphic $A_{l}^{\prime}$ terms.

The structure of the box diagrams is more complicated as they carry 4 flavour indices. Their MI expansion is given in appendix E.5. All box diagram contributions decouple at least as $v^{2} / M^{2}$.

Calculating consistently the quantities $F_{L L}, \ldots, F_{B L R}^{\prime}$ to the order $v^{2} / M^{2}$ is not trivial for chargino and neutralino contributions. If the MI expansion is used only for the sfermion mass matrices but the calculations for the supersymmetric fermions are done in the mass eigenbasis, the direct dependence on the Lagrangian parameters is hidden and the decoupling properties of the amplitude cannot be seen directly. However, one can also treat the off-diagonal entries of the chargino and neutralino mass matrices as "mass insertions". With such an approach, the final result is expressed explicitly in terms of Lagrangian parameters, but the computations can get very complicated. At the order $v^{2} / M^{2}$ one needs to include diagrams with all combinations of two fermionic mass insertions (each providing one power of $v / M_{1}, v / M_{2}$ or $v / \mu$ ) or flavour diagonal slepton terms originating from trilinear $A$-terms (providing powers of $v A_{l} / M^{2}, v A_{l}^{\prime} / M^{2}$ ). Thus, to obtain an expansion of the $F$ 's in eq. (4.2), one needs to formally go to the 3rd order of MI expansion, adding all diagrams with up to two flavour conserving and one flavour violating mass insertion. Therefore, the number of diagrams grows quickly with the order of the expansion and such a method is tedious and prone to calculational mistakes.

In our paper, we employ a recently developed technique using a purely algebraic MI expansion of the ME amplitudes listed in section 3, without the need for direct diagrammatic MI calculations ("FET theorem") [31], automatised in the specialised MassToMI Mathematica package $[32,83]$. The use of this package and full automation of the calculations 
allows us to perform the required 3rd order MI expansion for a completely general SUSY mass spectrum, without making any simplifying assumptions. Such a result would be very difficult to obtain diagrammatically, as in the intermediate steps of the calculations (before accounting for the cancellations and simplifications between various contributions) the expressions may contain up to tens of thousand terms, even if the final results collected in appendix $\mathrm{E}$ are again relatively compact. In detail:

- We perform the expansion always up to the lowest non-vanishing order in the slepton LFV terms, taking into account the possible cancellations. Compared to previous analyses, we consider the non-holomorphic trilinear soft terms as well.

- In the MI expanded expressions we include all terms decreasing with the SUSY mass scale as $v^{2} / M_{\mathrm{SUSY}}^{2}$ (or slower), where $M_{\mathrm{SUSY}}$ denotes any of the relevant mass parameters in the MSSM Lagrangian (apart from the soft Higgs mass terms): diagonal soft slepton masses, gaugino masses $M_{1}, M_{2}$ or the $\mu$ parameter.

- We do not assume degeneracy or any specific hierarchy for the sleptons, sneutrinos or supersymmetric fermion masses.

- In calculating the LFV Higgs decays we keep the leading terms in the external Higgs boson mass $\left(m_{h}^{2} / M_{\text {SUSY }}^{2}\right)$.

The full set of the expanded expressions in the MI approximation for the photon, $Z^{0}$ and CP-even Higgs leptonic penguins and for the 4-lepton box diagrams is collected in appendix E.

We illustrate the accuracy of the derived MI formulae in figure 6. The plots show the ratio of the MI expanded couplings over the ones obtained in the mass eigenbasis with exact diagonalization. For this purpose, we start from the following setup where all mass parameters are given in $\mathrm{GeV}$ :

$$
\begin{aligned}
& \tan \beta=5 \quad m_{\tilde{\mu}_{L}}=300 \quad A_{\mu \mu}=A_{\mu \mu}^{\prime}=0.1 \sqrt{m_{\tilde{\mu}_{L}} m_{\tilde{\mu}_{R}}} \\
& \mu=200+100 i \quad m_{\tilde{\tau}_{L}}=330 \\
& M_{1}=150 \quad m_{\tilde{\mu}_{R}}=300 \quad A_{\tau \tau}=A_{\tau \tau}^{\prime}=0.1 \sqrt{m_{\tilde{\tau}_{L}} m_{\tilde{\tau}_{R}}} \\
& M_{2}=300 \quad m_{\tilde{\tau}_{R}}=350
\end{aligned}
$$

Next, to see the decoupling effects we scale this spectrum uniformly up to slepton masses of $2 \mathrm{TeV}$. For each of the six penguin Wilson coefficients describing the transition between 2nd and 3rd generation, $F_{\gamma L(R)}^{23}($ eq. $(2.2)), F_{Z L(R)}^{23}$ (eq. (2.7)) and $F_{h L}^{23} \equiv F_{h}^{232}, F_{h R}^{23} \equiv F_{h}^{322}$ (eq. (2.13)) we plot the quantity

$$
\Delta F=\left|\frac{F_{\mathrm{MI}}}{F_{\mathrm{ME}}}\right|-1
$$

as a function of the average slepton mass. The accuracy of left-handed (right-handed) Wilson coefficients is illustrated with red(blue) lines. As can be seen from figure 6 , the accuracy of MI expanded amplitudes is very good even for light SUSY particles and for $M_{\text {SUSY }}>500 \mathrm{GeV}$ always better than $95 \%$. 

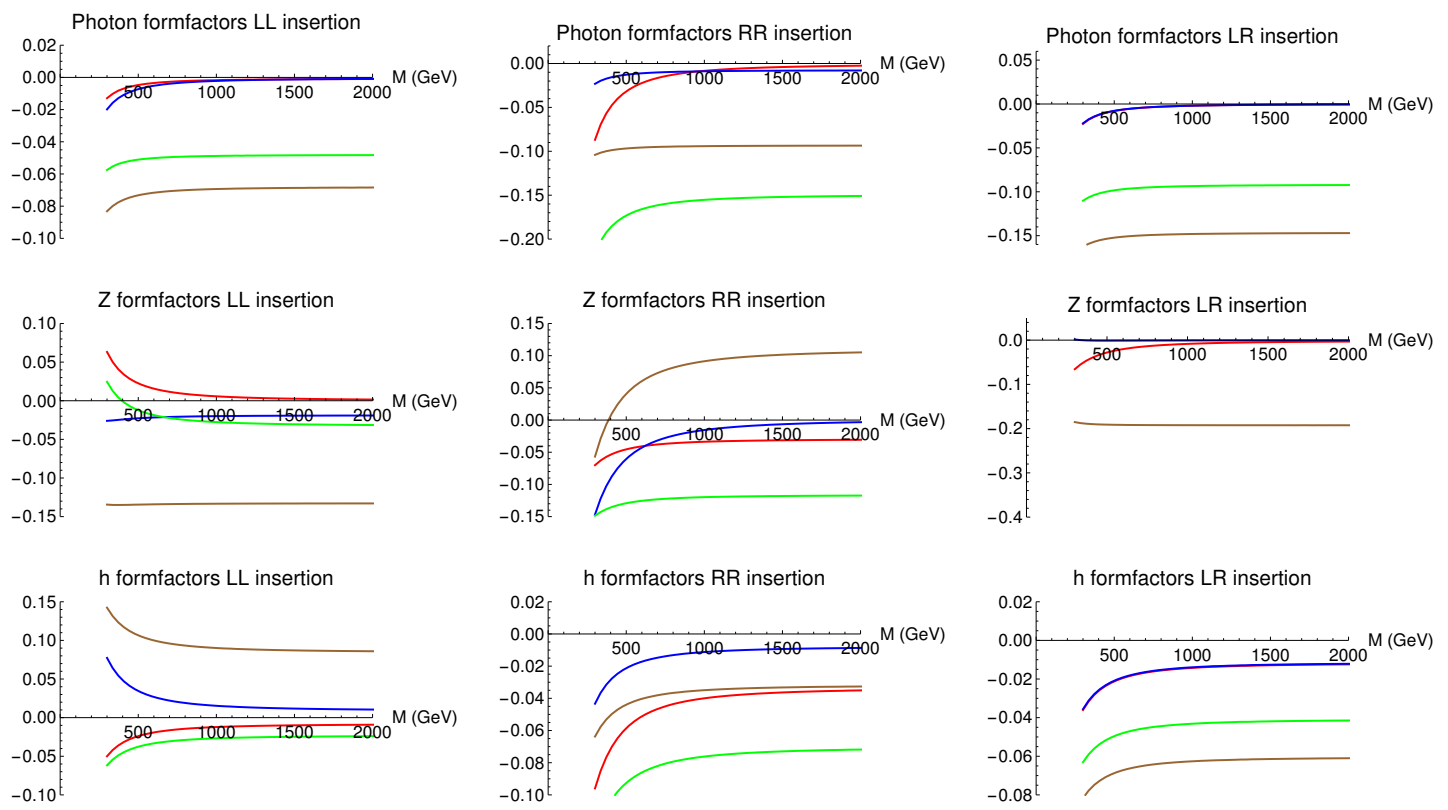

Figure 6. Accuracy of MI expansion for the penguin amplitudes. The curves show the ratio defined in eq. (4.4). Red and blue lines: $\Delta F$ for left and right couplings assuming a spectrum of eq. (4.3) for both the MI and ME expressions. Brown and green lines: $\Delta F$ (again for left and right couplings, respectively) assuming spectrum (4.3) for ME expressions but an universal degenerate sfermion mass in MI expressions. This assumption is inconsistent with non-zero off-diagonal elements of the mass matrices, which imply non-degenerate mass eigenstates. The plots show that the associated error can be numerically sizeable. The average SUSY mass scale $M$ (assumed to be equal to $\left.M_{2}=m_{\tilde{\mu}_{L}}=m_{\tilde{\mu}_{R}}\right)$ is shown on the horizontal axis.

Many analyses published to date for simplicity did not include the complete set of the contributions scaling like $v / M$ order and/or assumed a partially or fully degenerate SUSY spectrum. This procedure is inconsistent with non-zero off-diagonal elements of mass matrices, because the latter enforce unequal eigenvalues of the corresponding mass matrix. To illustrate the numerical effects arising from the incorrect neglection of SUSY mass splitting we plot the ratio of our expressions in the MI approximation for penguin Wilson coefficients calculated for degenerate slepton masses (equal to $300 \mathrm{GeV}$ rescaled by a common factor; other parameters as in eq. (4.3)) and the exact mass eigenbasis formulae (calculated with non-degenerate sfermion spectrum of eq. (4.3)) in figure 6. The accuracy of left-handed (right-handed) MI expanded Wilson coefficients with degenerate slepton spectrum is shown in green(brown). In this case discrepancy is much larger, of the order of 10\%-40\%, and does not disappear when increasing the total SUSY scale.

Some papers on the LFV in the MSSM, like e.g. refs. [7, 84], deal with general SUSY spectra. In order to compare the accuracy of the MI approximation derived in our analysis with previous works, we plotted in figure 7 the ratios of $\operatorname{Br}(\tau \rightarrow \mu \gamma)$ and $\operatorname{Br}(h \rightarrow \tau \mu)$ calculated using the exact (ME) and MI-expanded formulae scanning over randomly chosen MSSM mass spectra. In particular, in figure 7 we assume $\tan \beta=5, \alpha-\beta=-\pi / 2-\pi / 100$, $m_{h}=125 \mathrm{GeV}$, diagonal $A$ terms which are proportional to the lepton Yukawa couplings 

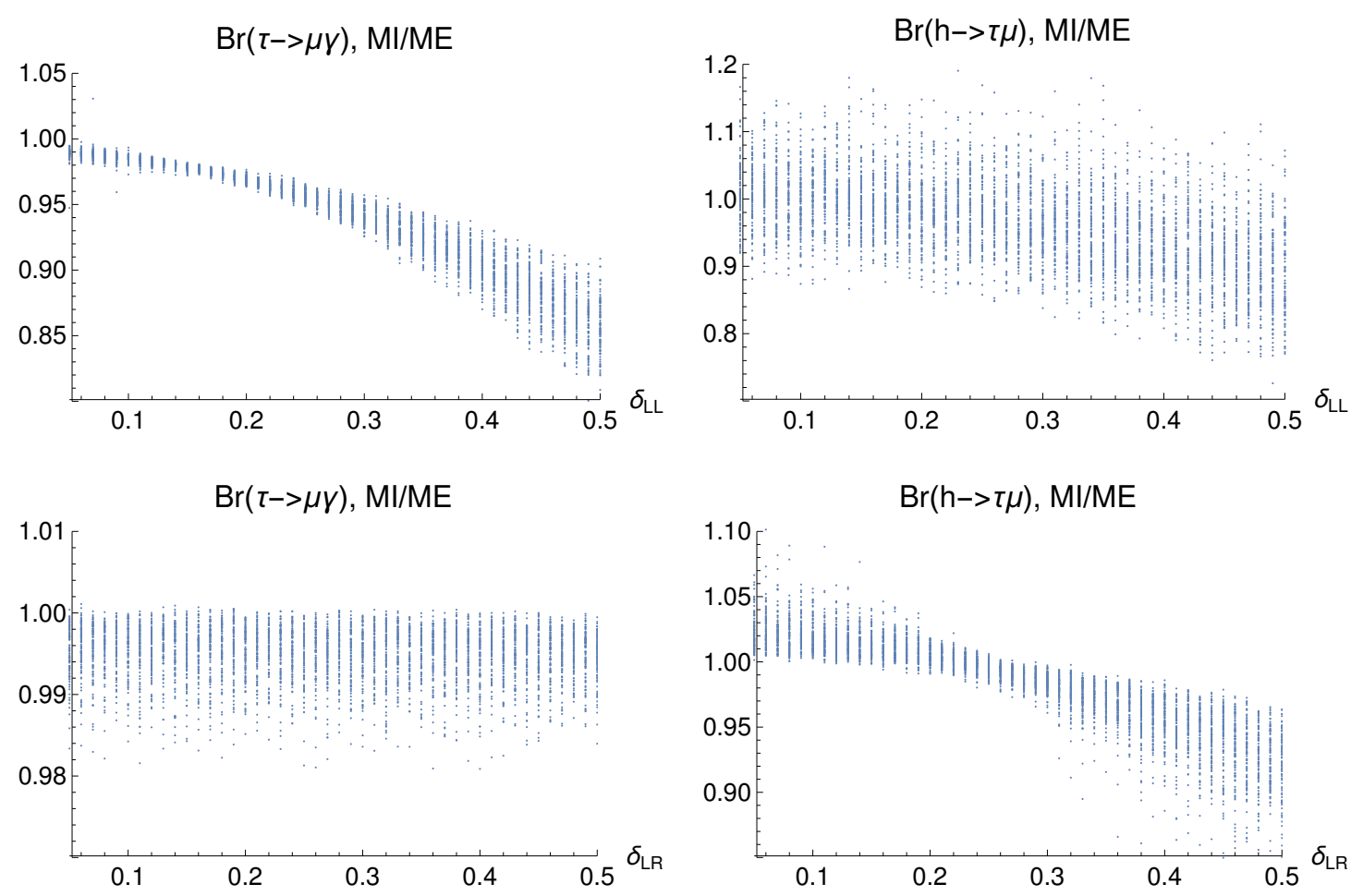

Figure 7. Accuracy of MI expansion for the $\tau \rightarrow \mu \gamma$ and $h \rightarrow \tau \mu$ decay rates. Points show the ratio $\operatorname{Br}(\tau \rightarrow \mu \gamma)^{M I} / \operatorname{Br}(\tau \rightarrow \mu \gamma)^{M E}$ (lower and upper left panel) and $\operatorname{Br}(h \rightarrow \tau \mu)^{M I} / \operatorname{Br}(h \rightarrow \tau \mu)^{M E}$ (lower and upper right panel) as a function of LL and LR mass insertion for $\tan \beta=5$ and random choice of other model parameters (see eq. (4.5)).

$\left(A_{l}^{I I}=A_{l}^{\prime I I}=Y_{l}^{I}\left(\left(M_{L L}^{2}\right)_{I I}\left(M_{R R}^{2}\right)_{J J}\right)^{1 / 4}\right)$ and we vary the mass parameters randomly and independently in the following ranges (all values are given in $\mathrm{GeV}$ and we set $M_{1}=M_{2} / 2$ ):

$$
M_{A} \in(200,500) \quad \quad \mu, M_{2}, m_{\tilde{\tau}_{L}}, m_{\tilde{\mu}_{L}}, m_{\tilde{\tau}_{R}}, m_{\tilde{\tau}_{R}} \in(500,1000) .
$$

As can be seen from upper left panel of figure 7, even for $\Delta_{L L}^{32}=0.5$ the accuracy of our MI expansion is better than about $15 \%$. This can be compared with the corresponding right panel of figure 8 in ref. [7] - there the difference between MI and ME calculation for the same value of $\Delta_{L L}^{32}=0.5$ is $20 \%-70 \%$, also the spread of points around the parabolic shape arising from neglected $\left(\Delta_{L L}^{32}\right)^{2}$ terms is much larger, $50 \%$ against a maximum of $10 \%$ in our approach. It is worth noting that the agreement in the lower left plot of figure 7 is almost perfect everywhere, which could be attributed to the fact that terms of higher order in $\Delta_{L R}$ are suppressed by additional $v / M$ powers (when $A$ terms are scaled linearly with $M$, like we choose) and thus small even for large $\Delta_{L R}$ values.

Accuracy of our MI expansion for $B r(h \rightarrow \tau \mu)$ shown in the right upper panel of figure 7 is worse, up to $20 \%$, because we include only non-decoupling $L L$ terms in our formulae, which is not a fully satisfying approximation for a SUSY scale in the range of 500-1000 GeV. Concerning the expansion in $\Delta_{L R}^{32}$ (where only decoupling terms contribute), we include them consistently and the accuracy is much better. This can be compared with ref. [84], 


\begin{tabular}{|l|r|r|r|r|}
\hline & Experimental upper bound & CL & Future sensitivity & CL \\
\hline$\tau \rightarrow e \gamma$ & $3.3 \times 10^{-8}[85]$ & $90 \%$ & $10^{-9}[86,87]$ & $90 \%$ \\
$\tau \rightarrow \mu \gamma$ & $4.4 \times 10^{-8}[85,88]$ & $90 \%$ & $10^{-9}[86]$ & $90 \%$ \\
$\mu \rightarrow e \gamma$ & $5.7 \times 10^{-13}[89]$ & $90 \%$ & $6 \times 10^{-14}[90]$ & $90 \%$ \\
\hline$Z \rightarrow \mu e$ & $7.5 \times 10^{-7}[91]$ & $95 \%$ & & \\
$Z \rightarrow \mu \tau$ & $1.2 \times 10^{-5}[92]$ & $95 \%$ & & \\
$Z \rightarrow \tau e$ & $9.8 \times 10^{-6}[92]$ & $95 \%$ & & \\
\hline$\mu \rightarrow e^{-} e^{+} e^{-}$ & $1.0 \times 10^{-12}[93]$ & $90 \%$ & $10^{-16}[94,95]$ & $90 \%$ \\
$\tau \rightarrow e^{-} e^{+} e^{-}$ & $2.7 \times 10^{-8}[96]$ & $90 \%$ & & \\
$\tau \rightarrow \mu^{-} \mu^{+} \mu^{-}$ & $2.1 \times 10^{-8}[96]$ & $90 \%$ & & \\
$\tau \rightarrow e^{-} \mu^{+} \mu^{-}$ & $2.7 \times 10^{-8}[96]$ & $90 \%$ & & \\
$\tau \rightarrow e^{+} \mu^{-} \mu^{-}$ & $1.7 \times 10^{-8}[96]$ & $90 \%$ & & \\
$\tau \rightarrow \mu^{-} e^{+} e^{-}$ & $1.8 \times 10^{-8}[96]$ & $90 \%$ & & \\
$\tau \rightarrow \mu^{+} e^{-} e^{-}$ & $1.5 \times 10^{-8}[96]$ & $90 \%$ & & \\
\hline$h \rightarrow e \tau$ & $6.1 \times 10^{-3}[97]$ & $90 \%$ & & \\
$h \rightarrow \mu \tau$ & $2.5 \times 10^{-3}[97]$ & $90 \%$ & & \\
$h \rightarrow \mu e$ & $3.6 \times 10^{-4}[98]$ & $90 \%$ & & \\
\hline$(\mu \rightarrow e)_{A u}$ & $7.0 \times 10^{-13}[99]$ & $90 \%$ & & \\
$(\mu \rightarrow e)_{A l}$ & \multicolumn{3}{|c|}{$50 \%]$} & \\
\hline
\end{tabular}

Table 2. Upper bounds on LFV decays of charged leptons. $h$ denotes the SM-like Higgs boson.

considering the same process. The agreement for $\Delta_{L L}^{32}=0.5$ in upper left panel of figure 6 ("general scenario") in [84] is better than ours, as they consistently include all $L L$ terms, not just non-decoupling ones. However, for $\Delta_{L R}^{32}$ (lower left panel of figure 6 in [84]) numerical accuracy of our formulae seems to be similar or even better. In general, no significant deviations should be expected here, as for this process our approach and the analysis of ref. [84] are equivalent up to the chosen calculational technique (FET vs. diagrammatic MI calculation) and, eventually, the selection of the included or neglected contributions.

\section{Phenomenological analysis}

\subsection{Generic bounds on LFV parameters}

As outlined in the introduction, flavour violation in the charged lepton sector is strongly constrained experimentally. In table 2 we collect the current and expected future experimental bounds on the processes discussed so far.

Assuming the absence of fine-tuned cancellations between different flavour violating parameters, the order of magnitude of the bounds on a given flavour violating entry $\Delta$ can be obtained by assuming that it is the only source of flavour violation. At the lowest order 


\begin{tabular}{|l|c|c|c|c|c|c|c|}
\hline Process & $(I, J)$ & $\Delta_{L L}^{I J}$ & $\Delta_{R R}^{I J}$ & $\Delta_{L R}^{I J}$ & $\Delta_{R L}^{I J}$ & $\Delta_{L R}^{\prime I J}$ & $\Delta_{R L}^{\prime I J}$ \\
\hline $\tan \beta=2$ & \multicolumn{7}{|c|}{} \\
\hline$\mu \rightarrow e \gamma$ & $(2,1)$ & $8.4 \cdot 10^{-4}$ & $5.0 \cdot 10^{-3}$ & $8.4 \cdot 10^{-6}$ & $8.3 \cdot 10^{-6}$ & $4.1 \cdot 10^{-6}$ & $4.1 \cdot 10^{-6}$ \\
$\tau \rightarrow \mu \gamma$ & $(3,2)$ & $5.3 \cdot 10^{-1}$ & $\mathcal{O}(1)$ & $9.1 \cdot 10^{-2}$ & $9.1 \cdot 10^{-2}$ & $4.5 \cdot 10^{-2}$ & $4.5 \cdot 10^{-2}$ \\
$\tau \rightarrow e \gamma$ & $(3,1)$ & $4.6 \cdot 10^{-1}$ & $\mathcal{O}(1)$ & $7.8 \cdot 10^{-2}$ & $7.8 \cdot 10^{-2}$ & $3.9 \cdot 10^{-2}$ & $3.8 \cdot 10^{-2}$ \\
\hline $\tan \beta=20$ & $(2,1)$ & $1.0 \cdot 10^{-4}$ & $4.5 \cdot 10^{-4}$ & $7.5 \cdot 10^{-5}$ & $7.4 \cdot 10^{-5}$ & $3.7 \cdot 10^{-6}$ & $3.7 \cdot 10^{-6}$ \\
\hline$\mu \rightarrow e \gamma$ & $(3,2)$ & $6.5 \cdot 10^{-2}$ & $2.9 \cdot 10^{-1}$ & $8.2 \cdot 10^{-1}$ & $8.2 \cdot 10^{-1}$ & $4.0 \cdot 10^{-2}$ & $4.0 \cdot 10^{-2}$ \\
$\tau \rightarrow \mu \gamma$ & $(3,1)$ & $5.7 \cdot 10^{-2}$ & $2.5 \cdot 10^{-1}$ & $7.0 \cdot 10^{-1}$ & $7.0 \cdot 10^{-1}$ & $3.4 \cdot 10^{-2}$ & $3.4 \cdot 10^{-2}$ \\
$\tau \rightarrow e \gamma$ &
\end{tabular}

Table 3. Upper bounds on the LFV parameters $\Delta$ from radiative charged lepton decays for the MSSM spectrum defined in eq. (5.3) and a SUSY scale of $M=400 \mathrm{GeV}$. All bounds scale (i.e. weaken) like $M^{2}$.

in the MI expansion, any LFV observable $X$ scales like $\Delta^{2}$ :

$$
X \approx f\left(m_{1}, \ldots, m_{n}\right)|\Delta|^{2},
$$

where $f$ is a known (non-negative) function of diagonal mass parameters - for any given process it can be extracted from the expanded expressions listed in appendix E. Thus, the experimental bound on $\Delta$ from a given measurement can be written as:

$$
|\Delta| \leq \sqrt{\frac{X^{\exp }}{f\left(m_{1}, \ldots, m_{n}\right)}} \sqrt{\frac{X^{\text {future }}}{X^{\exp }}} \equiv \Delta\left(m_{1}, \ldots, m_{n}\right) \sqrt{\frac{X^{\text {future }}}{X^{\exp }}}
$$

where by $X^{\exp }$ we denote one of the current experimental bounds listed in section 5.1 and $X^{\text {future }}$ is the expected future sensitivity.

To estimate the order of magnitude of the bounds on all types of mass insertions, we assume a common mass scale $M$ for all flavour diagonal SUSY parameters:

$$
\begin{aligned}
m_{\tilde{e}_{L I}} & =m_{\tilde{e}_{R I}}=M_{1}=M_{2}=\mu=M_{A}=M, \\
A_{\ell}^{I I} & =A_{\ell}^{\prime I I}=Y_{\ell}^{I} M .
\end{aligned}
$$

Currently, the strongest bounds on the dimensionless LFV parameters $\Delta$ defined in eq. (4.2) originate from the radiative lepton decays $\ell \rightarrow \ell^{\prime} \gamma$. We list such bounds for the parameter setup defined in eq. (5.3) and for the SUSY scale of $M=400 \mathrm{GeV}$ in table 3.

The 3-body decays of charged lepton lead to bounds which are approximately one order of magnitude weaker. In table 4 we display the relative strength of such bounds comparing them to the ones obtained from the radiative lepton decays, i.e. the ratios of bounds from radiative decays over the ones from 3-body decays. Such ratios remain constant with increasing $M$ up to the scale where the non-decoupling Higgs penguin contributions start to contribute. However, such effects occur for $M \gtrsim 30 \mathrm{TeV}$ for $\tau^{ \pm} \rightarrow \mu^{ \pm} \mu^{ \pm} \mu^{\mp}$ and $\tau^{ \pm} \rightarrow e^{ \pm} \mu^{ \pm} \mu^{\mp}$ decays and for even higher $M$ for the decays with electron pair in the 


\begin{tabular}{|l|c|c|c|c|c|c|c|}
\hline Process & $(I, J)$ & $\Delta_{L L}^{I J}$ & $\Delta_{R R}^{I J}$ & $\Delta_{L R}^{I J}$ & $\Delta_{R L}^{I J}$ & $\Delta_{L R}^{\prime I J}$ & $\Delta_{R L}^{\prime I J}$ \\
\hline $\tan \beta=2$ & \multicolumn{7}{|c|}{} \\
\hline$\mu \rightarrow e e e$ & $(2,1)$ & $1.7 \cdot 10^{+1}$ & $1.5 \cdot 10^{+1}$ & $1.6 \cdot 10^{+1}$ & $1.6 \cdot 10^{+1}$ & $1.6 \cdot 10^{+1}$ & $1.6 \cdot 10^{+1}$ \\
$\tau \rightarrow \mu \mu \mu$ & $(3,2)$ & $1.5 \cdot 10^{+1}$ & $1.2 \cdot 10^{+1}$ & $1.4 \cdot 10^{+1}$ & $1.4 \cdot 10^{+1}$ & $1.4 \cdot 10^{+1}$ & $1.4 \cdot 10^{+1}$ \\
$\tau \rightarrow \mu e^{+} e^{-}$ & $(3,2)$ & $1.3 \cdot 10^{+1}$ & $1.1 \cdot 10^{+1}$ & $1.2 \cdot 10^{+1}$ & $1.2 \cdot 10^{+1}$ & $1.2 \cdot 10^{+1}$ & $1.2 \cdot 10^{+1}$ \\
$\tau \rightarrow e e e$ & $(3,1)$ & $8.6 \cdot 10^{+0}$ & $8.2 \cdot 10^{+0}$ & $8.5 \cdot 10^{+0}$ & $8.5 \cdot 10^{+0}$ & $8.5 \cdot 10^{+0}$ & $8.5 \cdot 10^{+0}$ \\
$\tau \rightarrow e \mu^{+} \mu^{-}$ & $(3,1)$ & $6.9 \cdot 10^{+0}$ & $6.7 \cdot 10^{+0}$ & $6.8 \cdot 10^{+0}$ & $6.8 \cdot 10^{+0}$ & $6.8 \cdot 10^{+0}$ & $6.8 \cdot 10^{+0}$ \\
\hline $\tan \beta=20$ & \multicolumn{7}{|c|}{} \\
\hline$\mu \rightarrow e e e$ & $(2,1)$ & $1.6 \cdot 10^{+1}$ & $1.6 \cdot 10^{+1}$ & $1.6 \cdot 10^{+1}$ & $1.6 \cdot 10^{+1}$ & $1.6 \cdot 10^{+1}$ & $1.6 \cdot 10^{+1}$ \\
$\tau \rightarrow \mu \mu \mu$ & $(3,2)$ & $1.4 \cdot 10^{+1}$ & $1.4 \cdot 10^{+1}$ & $1.4 \cdot 10^{+1}$ & $1.4 \cdot 10^{+1}$ & $1.4 \cdot 10^{+1}$ & $1.4 \cdot 10^{+1}$ \\
$\tau \rightarrow \mu e^{+} e^{-}$ & $(3,2)$ & $1.3 \cdot 10^{+1}$ & $1.2 \cdot 10^{+1}$ & $1.2 \cdot 10^{+1}$ & $1.2 \cdot 10^{+1}$ & $1.2 \cdot 10^{+1}$ & $1.2 \cdot 10^{+1}$ \\
$\tau \rightarrow e e e$ & $(3,1)$ & $8.5 \cdot 10^{+0}$ & $8.5 \cdot 10^{+0}$ & $8.5 \cdot 10^{+0}$ & $8.5 \cdot 10^{+0}$ & $8.5 \cdot 10^{+0}$ & $8.5 \cdot 10^{+0}$ \\
$\tau \rightarrow e \mu^{+} \mu^{-}$ & $(3,1)$ & $6.8 \cdot 10^{+0}$ & $6.8 \cdot 10^{+0}$ & $6.8 \cdot 10^{+0}$ & $6.8 \cdot 10^{+0}$ & $6.8 \cdot 10^{+0}$ & $6.8 \cdot 10^{+0}$ \\
\hline
\end{tabular}

Table 4. Ratios of upper bounds on the LFV parameters $\Delta$ from the searches for 3-body and radiative decays of charged leptons. The MSSM spectrum is defined in eq. (5.3).

final state. For such a large $M$ the branching ratios for all 3-body decays are, anyway, below the current experimental sensitivities even for $\mathcal{O}\left(\Delta^{I J}\right) \sim 1$.

We do not display the bounds from LFV violating $Z^{0}$ decays as they are much weaker (3 to 8 orders of magnitude depending on which parameter $\Delta$ is chosen). This can be attributed to the large $Z$ boson width - for comparable $\Gamma\left(Z \rightarrow \ell \ell^{\prime}\right)$ and $\Gamma\left(\ell \rightarrow \ell^{\prime} \gamma\right)$ partial decay widths the difference in total widths leads to $\operatorname{Br}\left(\ell \rightarrow \ell^{\prime} \gamma\right) \gg \operatorname{Br}\left(Z \rightarrow \ell \ell^{\prime}\right)$. Thus, bounds from $\operatorname{Br}\left(Z \rightarrow \ell \ell^{\prime}\right)$ are not competitive (nor they will be in the foreseeable future) compared to those from other observables.

As can be seen in table 5 , the bounds on $\Delta$ parameters from LFV flavour Higgs boson decay searches are much weaker than those from the radiative charged lepton decays. However, in the Higgs sector some effects proportional to lepton Yukawa couplings or to the non-holomorphic terms are non-decoupling and are not weakened by increasing $M$ like other contributions, for fixed Higgs sector parameters. In table 5 we assume

$$
\alpha-\beta=-\frac{\pi}{2}-\gamma,
$$

with $\gamma=\pi / 100$. Using the tree-level relations of the MSSM Higgs sector in the limit of $\tan \beta>1$ and small values of $\gamma$ one has

$$
M_{A}=M_{Z} \sqrt{\frac{\sin 2(\alpha+\beta)}{\sin 2(\alpha-\beta)}} \approx M_{Z} \sqrt{\frac{-\sin 4 \beta}{2 \gamma}},
$$

this corresponds to $M_{A} \sim 350 \mathrm{GeV}$ for $\tan \beta=2$ and $M_{A} \sim 190 \mathrm{GeV}$ for $\tan \beta=20$ (the exact value including loop corrections may vary, depending on the squark parameters which we do not specify here).

The bounds on $\Delta_{L L}^{I J}, \Delta_{R R}^{I J}, \Delta_{L R}^{\prime I J}, \Delta_{R L}^{\prime I J}$ from the leptonic Higgs boson decay would decouple only if also $M_{A}$ is scaled up simultaneously with SUSY particle masses (thus assuring 


\begin{tabular}{|l|c|c|c|c|c|c|c|}
\hline Process & $(I, J)$ & $\Delta_{L L}^{I J}$ & $\Delta_{R R}^{I J}$ & $\Delta_{L R}^{I J}$ & $\Delta_{R L}^{I J}$ & $\Delta_{L R}^{\prime I J}$ & $\Delta_{R L}^{\prime I J}$ \\
\hline $\tan \beta=2$ & \multicolumn{7}{|l|}{} \\
\hline$h \rightarrow \mu e$ & $(2,1)$ & $1.8 \cdot 10^{+7}$ & $1.7 \cdot 10^{+6}$ & $2.6 \cdot 10^{+7}$ & $2.6 \cdot 10^{+7}$ & $1.0 \cdot 10^{+7}$ & $1.0 \cdot 10^{+7}$ \\
$h \rightarrow \tau \mu$ & $(3,2)$ & $4.4 \cdot 10^{+3}$ & $3.8 \cdot 10^{+2}$ & $6.3 \cdot 10^{+3}$ & $6.3 \cdot 10^{+3}$ & $2.5 \cdot 10^{+3}$ & $2.5 \cdot 10^{+3}$ \\
$h \rightarrow \tau e$ & $(3,1)$ & $8.0 \cdot 10^{+3}$ & $7.5 \cdot 10^{+2}$ & $1.1 \cdot 10^{+4}$ & $1.1 \cdot 10^{+4}$ & $4.9 \cdot 10^{+3}$ & $4.9 \cdot 10^{+3}$ \\
\hline $\tan \beta=20$ & $(2,1)$ & $3.9 \cdot 10^{+6}$ & $8.3 \cdot 10^{+6}$ & $5.1 \cdot 10^{+7}$ & $5.1 \cdot 10^{+7}$ & $1.2 \cdot 10^{+6}$ & $1.2 \cdot 10^{+6}$ \\
\hline$h \rightarrow \mu e$ & $(3,2)$ & $9.5 \cdot 10^{+2}$ & $1.9 \cdot 10^{+3}$ & $1.3 \cdot 10^{+4}$ & $1.2 \cdot 10^{+4}$ & $2.9 \cdot 10^{+2}$ & $2.9 \cdot 10^{+2}$ \\
$h \rightarrow \tau \mu$ & $(3,1)$ & $1.7 \cdot 10^{+3}$ & $3.5 \cdot 10^{+3}$ & $2.3 \cdot 10^{+4}$ & $2.2 \cdot 10^{+4}$ & $5.3 \cdot 10^{+2}$ & $5.4 \cdot 10^{+2}$ \\
$h \rightarrow \tau e$ &
\end{tabular}

Table 5. Ratios of upper bounds on the LFV $\Delta$ parameters from leptonic Higgs boson decays and from radiative decays of charged leptons. The MSSM spectrum is defined in eq. (5.3) (with the exception of setting $A_{l}^{\prime I I}=0$ ) and a SUSY scale of $M=400 \mathrm{GeV}$. The ratios for $\Delta_{L L}^{I J}, \Delta_{R R}^{I J}, \Delta_{L R}^{\prime I J}$, $\Delta_{R L}^{\prime I J}$ decrease with $M^{2}$, assuming fixed masses and mixing angles in the Higgs sector.

that the Higgs decay rates do not violate the Appelquist-Carrazone theorem [101]). This interesting feature is discussed in more details in section 5.4.

\subsection{Dependence on the mass splitting}

The formulae derived in the previous sections allow to analyse how the bounds on LFV mass insertions depend on the splitting between different SUSY masses. However, any process involving transition between the generations $I$ and $J$ depends in general, even at lowest order in the flavour violating MI's, on many mass parameters: $\mu$, gaugino masses $M_{1}, M_{2}$, left and right diagonal slepton soft masses $m_{\tilde{e}_{L I}}, m_{\tilde{e}_{L J}}, m_{\tilde{e}_{R I}}, m_{\tilde{e}_{R J}}$, and for the Higgs decays also on $M_{A}$ or on $\alpha$ angle. To simplify the discussion, we only take into account the bounds from $\ell \rightarrow \ell^{\prime} \gamma$ decays, which are currently most constraining.

In figure 8 we illustrate the dependence of the upper bounds on the $\Delta$ parameters originating from $\mu \rightarrow e \gamma$ on the mass splitting between left and right-handed sleptons for

$$
\begin{aligned}
\tan \beta & =2, & \mu & =M_{1}=M_{2} \equiv M=800 \mathrm{GeV} \\
m_{\tilde{e}_{L}} & =m_{\tilde{\mu}_{L}}=m_{L}, & m_{\tilde{e}_{R}} & =m_{\tilde{\mu}_{R}}=m_{R}, \\
A_{\ell}^{e e} & =A_{\ell}^{\prime e e}=Y_{e} \sqrt{m_{L} m_{R}}, & A_{\ell}^{\mu \mu} & =A_{\ell}^{\prime \mu \mu}=Y_{\mu} \sqrt{m_{L} m_{R}} .
\end{aligned}
$$

We have chosen here an average SUSY mass scale of $M=800 \mathrm{GeV}$, higher than $M=$ $400 \mathrm{GeV}$ used in tables $3-5$, to avoid the experimental bounds on slepton masses even in the case of a large splitting between the left and right-handed masses.

The features of plots in figure 8 can be understood using the expanded expressions for effective photon couplings collected in appendix E.1. As an example, let us consider the interesting cancellation between different contributions in the case of $\Delta_{R R}^{12}$ (right upper panel of figure 8). For our parameter setup, the coefficient $X_{\gamma N 2}^{e \mu}$ multiplying the RR parameter (see eq. (E.4)) can be reduced to the form

$$
X_{\gamma N 2}^{e \mu}=\frac{v_{1} Y_{\mu}}{M^{2}} f\left(x_{L}, x_{R}\right),
$$



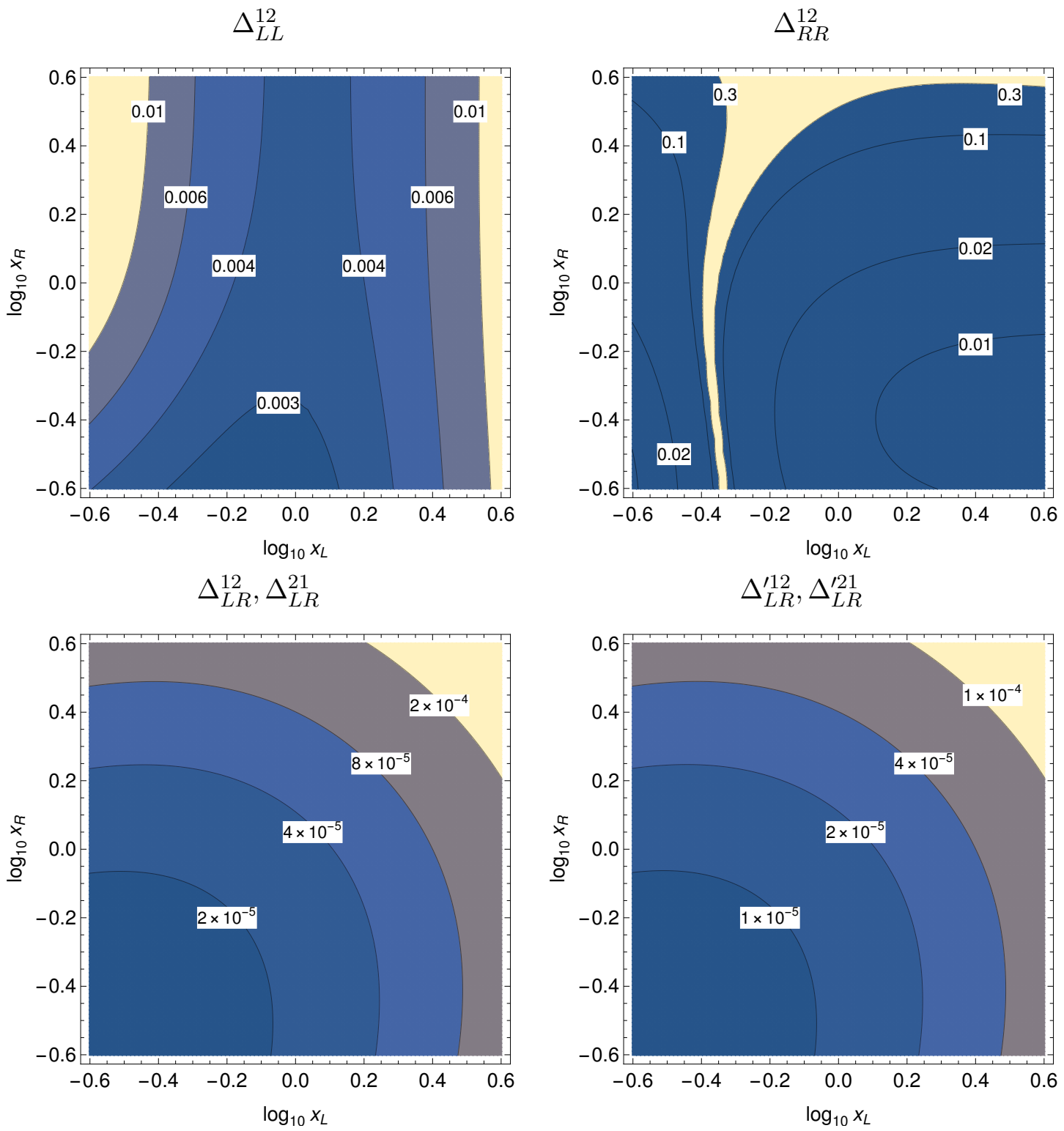

Figure 8. Upper bounds on the LFV parameters from $\mu \rightarrow e \gamma$ for $\tan \beta=2$ and $M=800 \mathrm{GeV}$ as a function of the splitting between the masses of gaugino and sleptons of different chiralities. The normalised slepton masses $x_{L(R)}=m_{L(R)} / M$ are plotted on the axes.

where $f\left(x_{L}, x_{R}\right)$ is a known, although complicated, dimensionless, rational and logarithmic function of mass ratios whose analytical form can be obtained using eq. (E.4), the loop integrals collected in appendix B and the definitions of divided differences from appendix $\mathrm{C}$. The properties of this function can be examined analytically and numerically. One finds

- For $x_{R}$ in the wide range $0.1-4$ the function $f$ vanishes for $x_{L} \sim 0.45$ (the exact value depends only weakly on $x_{R}$ ). As a result, the bounds on $\Delta_{R R}^{12}$ disappear completely for $m_{L} \sim 0.45 M$. 
- For large values of $x_{R} \gtrsim 5$ the position where the function $f$ becomes zero shifts towards bigger values of $x_{L}$. In addition, in this limit $f$ is suppressed by an overall factor $1 / x_{R}$, thus the bounds on $\Delta_{R R}^{12}$ become weaker for a larger values of $x_{R}$.

- For large values of $x_{L}$ the function $f$ depends on $x_{R}$ only. Therefore, the contour lines become horizontal.

- For small values of $x_{L}$ the function $f$ behaves like $1 / x_{L}$. Thus, the bounds on $\Delta_{R R}^{12}$ become stronger.

A similar analysis can be done for the bounds on $\Delta_{L L}^{12}$. However, the coefficient multiplying $\Delta_{L L}^{12}$ contains contributions from both chargino and neutralino loops and does not vanish for any mass pattern. Therefore, there is no cancellation area in the upper left panel of figure 8. In this case, the bound on $\Delta_{L L}^{12}$ is strongest for $m_{L} \sim M$ and $m_{R} \lesssim M$. For the case in which the left slepton masses are much lighter or much heavier than the masses of the SUSY fermion, the bounds become weaker.

Bounds on LR parameters, both holomorphic and non-holomorphic, are typically 12 orders of magnitude stronger than for LL and RR ones. In this case, the coefficient $X_{\gamma N 1}^{e \mu}$ multiplying the LR terms has a much simpler functional form. Therefore, it never vanishes and in addition is explicitly symmetric (as follows from the properties of divided differences) under the exchange of slepton mass arguments, as visible in both lower panels of figure 8. Furthermore, one can see that bounds on LR parameters are strongest for $m_{L}, m_{R} \lesssim M$ and become weaker when the slepton masses are much heavier than the chargino and neutralino masses. More quantitatively, $X_{\gamma N 1}^{e \mu}$ is proportional to the divided difference of the function $C_{12}$, which for $x \equiv x_{L}=x_{R}$ (corresponding to the diagonal of lower plots in figure 8) has the simple asymptotic behaviour

$$
C_{12}\left(\left\{m_{L}, m_{R}\right\}, M\right)=\left\{\begin{array}{cc}
-\frac{5}{2 M^{2}} & x \ll 1 \\
\frac{1}{2 M^{2} x^{2}} & x \gg 1
\end{array} .\right.
$$

From the form of eq. (5.7) it is immediately visible that the bounds become constant for small $x$ and fall like $1 / x^{2}$ for large $x$, as illustrated in the plots.

Using the formulae collected in appendix E, a similar discussion can be, if necessary, performed to explain the features or cancellation areas of other plots presented in this section. However, as the general analytical formulae in the MI approximation are rather complicated, we illustrate here other scenarios with numerical plots only.

Figure 9 shows similar bounds assuming identical left-and right-handed slepton masses which however differ among the generations, so that we choose

$$
\begin{aligned}
\tan \beta & =2, & \mu & =M_{1}=M_{2} \equiv M=800 \mathrm{GeV}, \\
m_{\tilde{e}_{L}} & =m_{\tilde{e}_{R}}=m_{\tilde{e}} & m_{\tilde{\mu}_{L}} & =m_{\tilde{\mu}_{R}}=m_{\tilde{\mu}}, \\
A_{\ell}^{e e} & =A_{\ell}^{\prime e e}=Y_{e} m_{\tilde{e}}, & A_{\ell}^{\mu \mu} & =A_{\ell}^{\prime \mu \mu}=Y_{\mu} m_{\tilde{\mu}},
\end{aligned}
$$

and plot the results in terms of $x_{e}=m_{\tilde{e}} / M$ and $x_{\mu}=m_{\tilde{\mu}} / M$. Again, a cancellation only exists for the bounds on $\Delta_{R R}^{12}$, for an almost constant ratio $m_{\tilde{\mu}} \sim 2.5 M$. In this case, the 

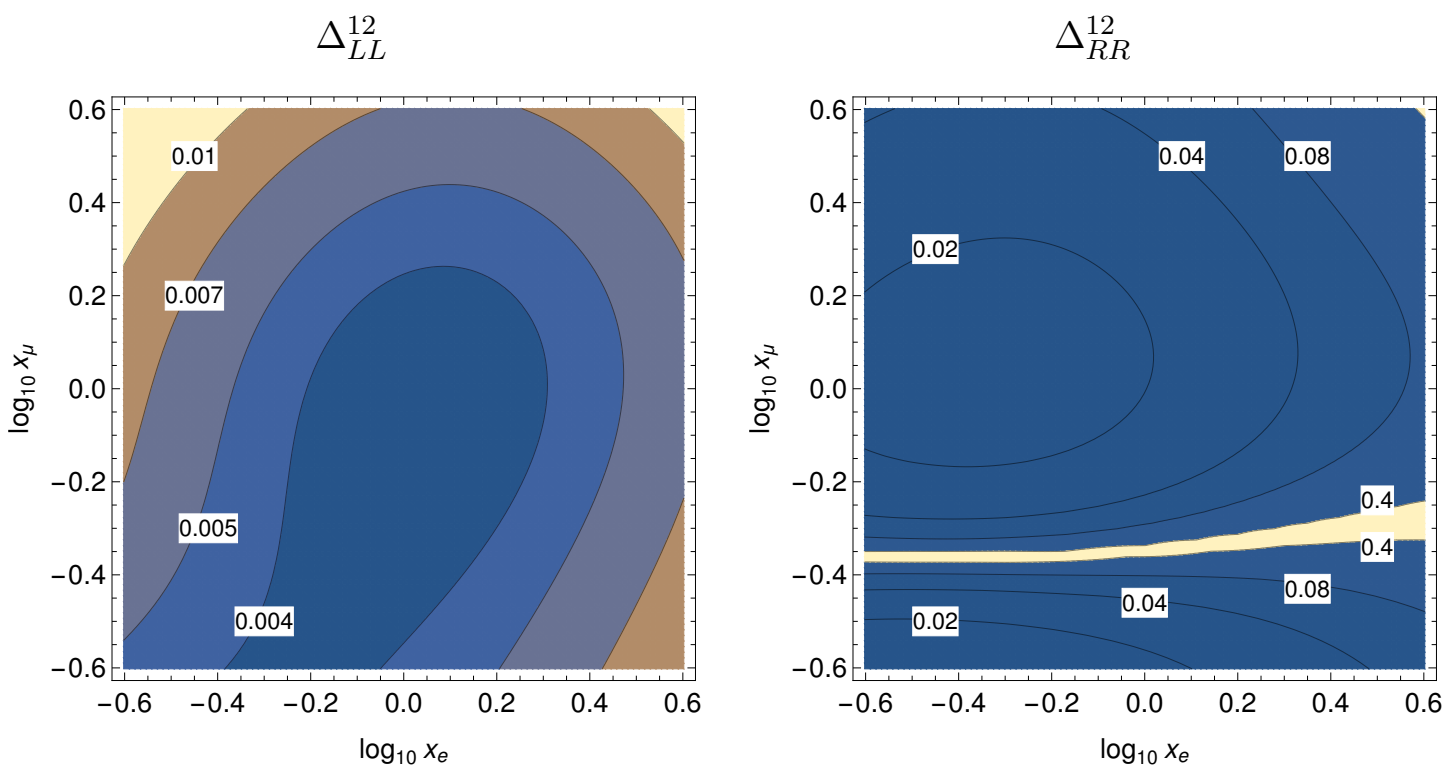

Figure 9. Upper bounds on the flavour violating LL and RR parameters using the current experimental limit on $\operatorname{Br}(\mu \rightarrow e \gamma)$ for $\tan \beta=2$ and $M=800 \mathrm{GeV}$ as a function of splitting between the masses of gaugino and sleptons of various flavours. The normalised selectron and smuon masses, $x_{e(\mu)}=m_{\tilde{e}(\tilde{\mu})} / M$ are plotted on the axes.

bounds on $\Delta_{L L}^{12}$ are strongest for small splitting between slepton and SUSY fermion masses, while the bounds on $\Delta_{R R}^{12}$ are, apart from the cancellation region, stronger for $m_{\tilde{\mu}} \lesssim M$. It is obvious from the form of $X_{\gamma N 1}^{e \mu}$ in eq. (E.4) that the bounds on the LR parameters, both holomorphic and non-holomorphic, have an identical behaviour as in the case of the $m_{L}-m_{R}$ splitting plotted in figure 8, with the replacements $x_{L} \leftrightarrow x_{e}, x_{R} \leftrightarrow x_{\mu}$.

Finally in figure 10 we assume an identical mass of $m=400 \mathrm{GeV}$ for all sleptons but vary $M_{1}=M_{2}$ and $\mu$. The results are displayed as a function of $x_{2}=M_{2} / M, x_{\mu}=\mu / M$ (we do not plot small values of $|\mu|<100 \mathrm{GeV}$ which are excluded by the direct searches for charginos and neutralinos). The structure of cancellation areas is more complicated, but again the "blind spots", where the bounds on MI's disappear, exist only for $\Delta_{R R}^{12}$. As expected from the form of $X_{\gamma N 1}^{e \mu}$ in eq. (E.4), the bounds on $\Delta_{L R}^{12}, \Delta_{L R}^{\prime 12}$ are at leading order independent of the $\mu$ parameter. They are also correlated with the bounds displayed in lower plots of figure 8, as for a fixed slepton mass and varied $M_{2}$ the coefficient $X_{\gamma N 1}^{e \mu}$ is now proportional to

$$
C_{12}\left(\{m, m\}, M_{2}\right)=\left\{\begin{array}{cl}
\frac{1}{2 m^{2}} & x \ll 1 \\
-\frac{5}{2 m^{2} x_{2}^{2}} & x \gg 1
\end{array}\right.
$$

so that again the bounds saturate for small $x_{2}$ and fall like $1 / x_{2}^{2}$ in the opposite limit.

Similar plots constraining 13 and 23 mass insertions have almost identical shape; the bounds are just rescaled by constant factors. The bounds on $\Delta_{L L}^{13}$ and $\Delta_{R R}^{13}\left(\Delta_{L L}^{23}\right.$ and $\left.\Delta_{R R}^{23}\right)$ are approximately 550 (650) times weaker than the bounds on $\Delta_{L L}^{12}$ and $\Delta_{R R}^{12}$, respectively. 

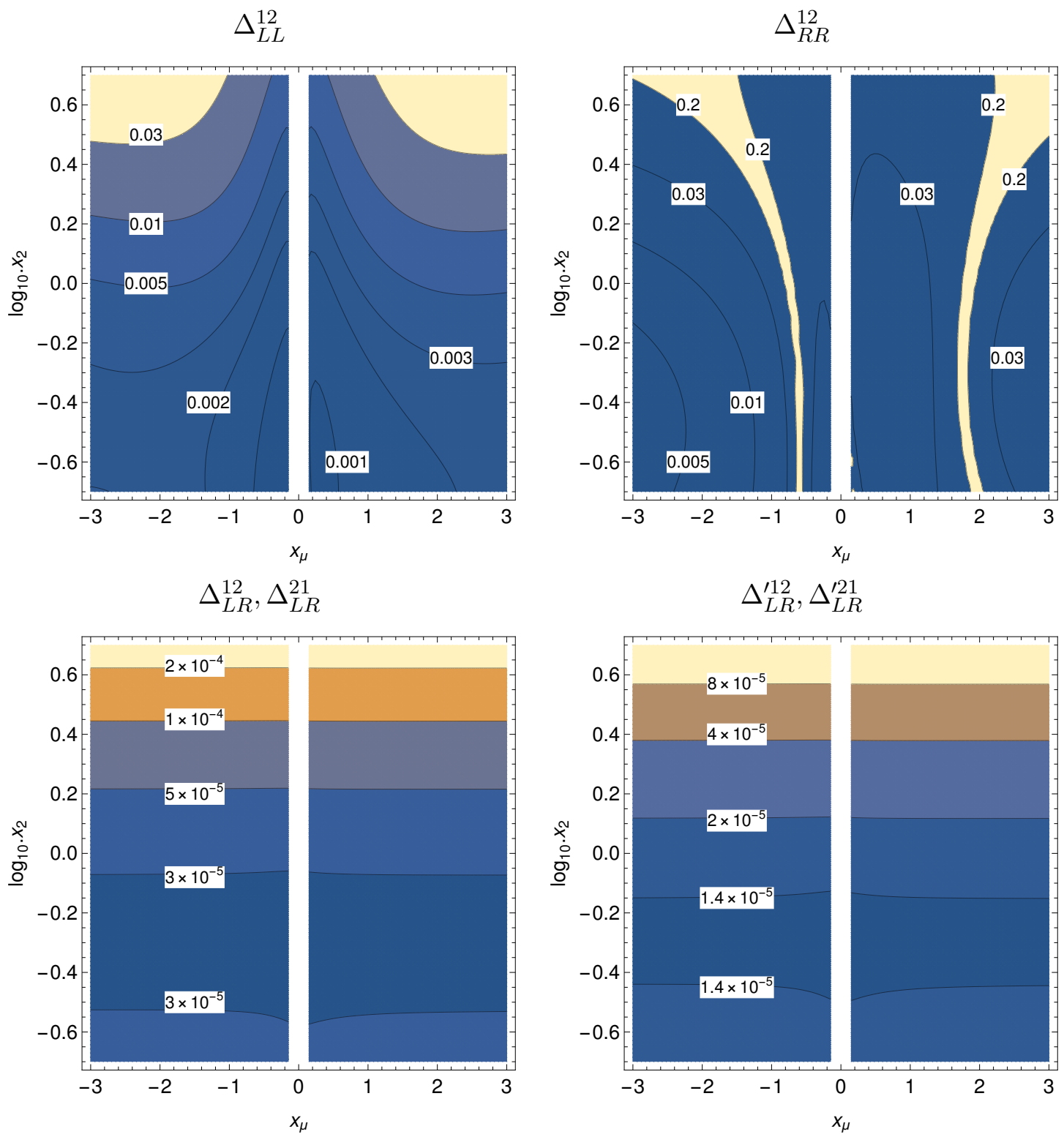

Figure 10. Upper bounds on the LFV parameters using the current experimental limit on the $\operatorname{Br}(\mu \rightarrow e \gamma)$ for degenerate slepton masses $M=800 \mathrm{GeV}$ as a function of mass splitting between the gaugino and the $\mu$ related parameters, $x_{\mu}=\mu / M, x_{2}=M_{1} / M=M_{2} / M$.

The bounds on $\Delta_{L R}^{13(31)}$ and $\Delta_{L R}^{\prime 13(31)}\left(\Delta_{L R}^{23(32)}\right.$ and $\left.\Delta_{L R}^{\prime 23(32)}\right)$ are respectively 9000 (11000) times weaker than the bounds on $\Delta_{L R}^{12(21)}$ and $\Delta_{L R}^{\prime 12(21)}$.

\subsection{Correlations between LFV processes}

The correlations between various leptonic decays, in particular radiative and 3-body charged lepton decays, are important for designing new experiments searching for the LFV phenomena. In the photon penguin domination scenario the ratio of decay rates for both 
processes is given by the simple formula:

$$
\frac{\operatorname{Br}\left(\ell \rightarrow 3 \ell^{\prime}\right)}{\operatorname{Br}\left(\ell \rightarrow \ell^{\prime} \gamma\right)} \approx \frac{\alpha_{e m}}{3 \pi}\left(\log \frac{m_{\ell}^{2}}{m_{\ell^{\prime}}^{2}}-\frac{11}{4}\right) .
$$

In this case the decision which measurement is more promising depends purely on experimental accuracy achievable for each of them. However, other types of contributions, like $Z$-penguin and box diagrams, can modify the ratio (5.9). Such contributions may be particularly important for a "blind spot" scenario, like the weakened limit on $\Delta_{R R}$ for some ratios of slepton and gaugino masses.

In figure 11 we plot the quantity $R_{\ell \ell^{\prime}}$ defined as

$$
R_{\ell \ell^{\prime}}=\frac{\alpha_{e m}}{3 \pi}\left(\log \frac{m_{\ell}^{2}}{m_{\ell^{\prime}}^{2}}-\frac{11}{4}\right) \frac{\operatorname{Br}\left(\ell \rightarrow \ell^{\prime} \gamma\right)}{\operatorname{Br}\left(\ell \rightarrow 3 \ell^{\prime}\right)},
$$

as a function of the SUSY mass splittings, in the same scenarios as described in figure 8 and figure 9. We assume non-vanishing $\Delta_{L L}^{12}$ and $\Delta_{R R}^{12}$ terms. For LR terms, both holomorphic and non-holomorphic, a photon penguin dominated scenario is always realised and $R_{\ell \ell^{\prime}}$ is very close to 1.

As one can see from figure 11, radiative and 3-body decays are almost always closely correlated, with $R_{\ell \ell^{\prime}}$ differing from 1 by a few $\%$ at most. Exceptions are only possible for parameter combinations for which $\operatorname{Br}\left(\ell \rightarrow \ell^{\prime} \gamma\right)$ becomes small due to cancellations or some other type of suppression, like in scenarios with large mass splitting (compare figures 8 and 9). Simultaneously, $\operatorname{Br}\left(\ell \rightarrow 3 \ell^{\prime}\right)$ is given by the more complicated expression (3.11), which in the limit of small photon penguin contribution becomes the sum of positive terms and cannot vanish. Thus, although both decays are usually strongly correlated and only relative experimental sensitivities decide which of them has better chances to discover generic LFV effects mediated by the slepton sector, for some particular ranges of MSSM parameter searches for 3-body charged lepton decays are a safer choice, allowing to avoid blind spots appearing for such setups due to the suppression of $\ell \rightarrow \ell^{\prime} \gamma$ decay rates.

\subsection{Non-decoupling effects in LFV Higgs decays}

LFV Higgs decays in the SM are absent at the tree level and strongly suppressed also at the loop level. Examining LFV Higgs boson decays within the MSSM is very interesting because, contrary to other processes discussed in this paper, some contributions to the Higgs decay amplitudes proportional to the lepton Yukawa couplings or to the non-holomorphic trilinear slepton soft terms do not decouple in the limit of heavy SUSY masses and can be potentially large.

As can be seen from tables 5, for an average SUSY mass scale of $M=400 \mathrm{GeV}$ and the parameter setup of eq. (5.3) the upper bounds on the flavour violating parameters from Higgs decays are much weaker than from the other processes. However, the bounds from Higgs decays on the $\Delta_{L L}^{I J}, \Delta_{R R}^{I J}$ and on the non-holomorphic LR terms $\Delta_{L R}^{I J}$ do not scale like $1 / M^{2}$. Thus, comparing the limits on $\Delta_{L R}^{\prime 13}$ and $\Delta_{L R}^{\prime 23}$ entries from $h \rightarrow \ell \ell^{\prime}$ and $\ell \rightarrow \ell^{\prime} \gamma$ decays one can check that e.g. for $\tan \beta=20$ and

$$
M_{\mathrm{SUSY}} \gtrsim \frac{1.5}{\sqrt{|\cos (\alpha-\beta)|}} \mathrm{TeV}
$$



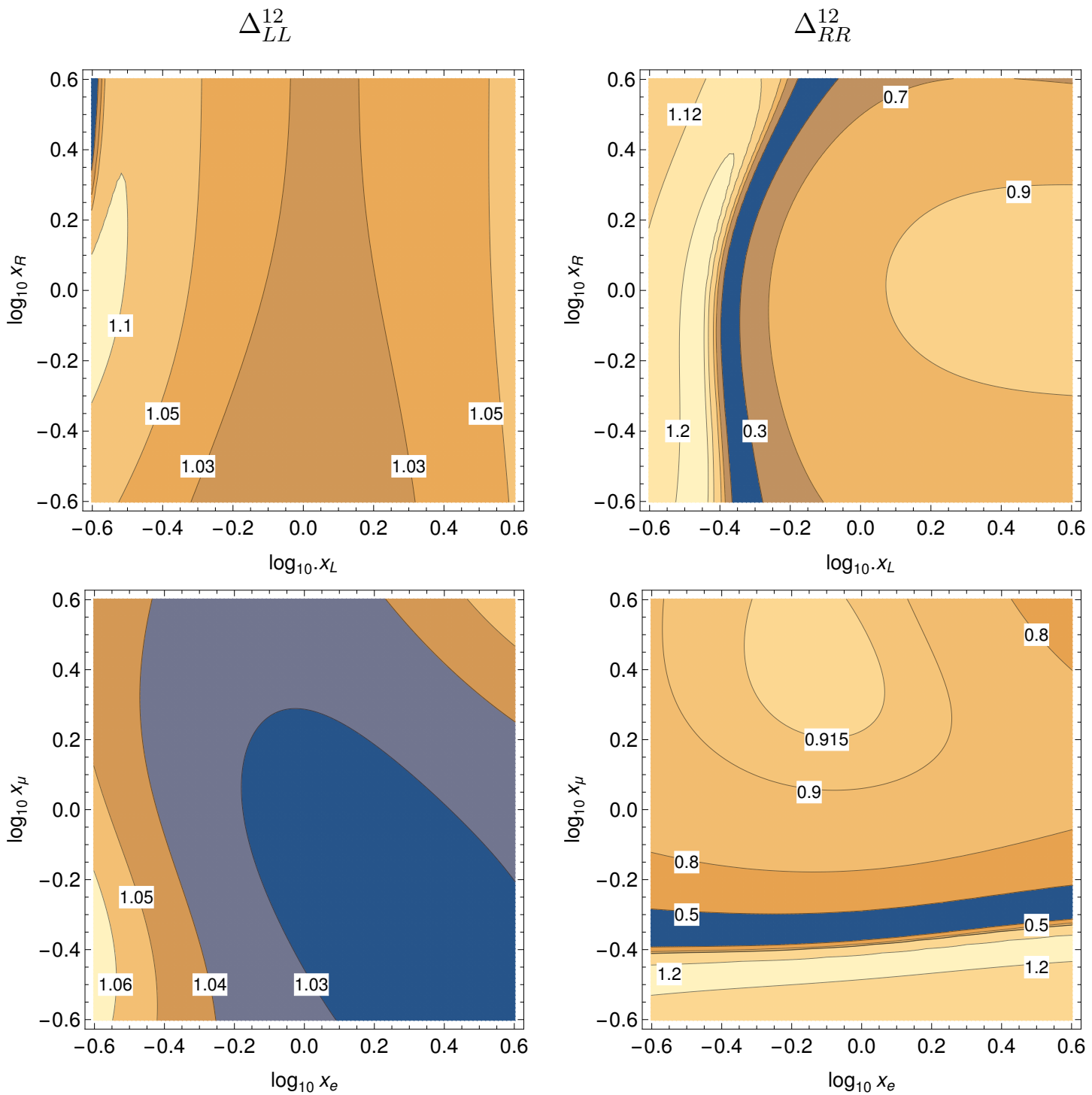

Figure 11. The ratio $R_{\ell \ell^{\prime}}$ as a function of the mass splitting between left and right slepton masses (upper row) and between the selectron and smuon masses (lower row) for $M=800 \mathrm{GeV}$ as a function of the normalised slepton masses $x_{L(R)}=m_{L(R)} / M$ and $x_{\tilde{e}(\tilde{\mu})}=m_{\tilde{e}(\tilde{\mu})} / M$.

the latter are becoming weaker. For $\Delta_{L R}^{\prime 12}$ the same occurs at a much higher scale

$$
M_{\mathrm{SUSY}} \gtrsim \frac{220}{\sqrt{|\cos (\alpha-\beta)|}} \mathrm{TeV} \text {. }
$$

The bounds on $\Delta_{L L}^{I J}, \Delta_{R R}^{I J}$ are obtained assuming that the flavour diagonal $A_{l}^{\prime}$ terms vanish, so that all non-decoupling LL and RR contributions are proportional to the Yukawa couplings (see eq. (E.23)). In this case the Higgs decays become most constraining for slightly higher SUSY scales, again for $\tan \beta=20$ and $\alpha$ angle of eq. (5.4) bounds on $\Delta_{L L}^{I J}$ and $\Delta_{R R}^{I J}$ from Higgs decays become stronger than the bounds from $\ell \rightarrow \ell^{\prime} \gamma$ decays for $M_{\mathrm{SUSY}} \gtrsim 2 / \sqrt{|\cos (\alpha-\beta)|} \mathrm{TeV}$ for $\tau \mu$ transitions, $M_{\mathrm{SUSY}} \gtrsim 3 / \sqrt{|\cos (\alpha-\beta)|} \mathrm{TeV}$ for $\tau e$ transitions and $M_{\text {SUSY }} \gtrsim 200 / \sqrt{|\cos (\alpha-\beta)|} \mathrm{TeV}$ for $\mu$ e transitions. 


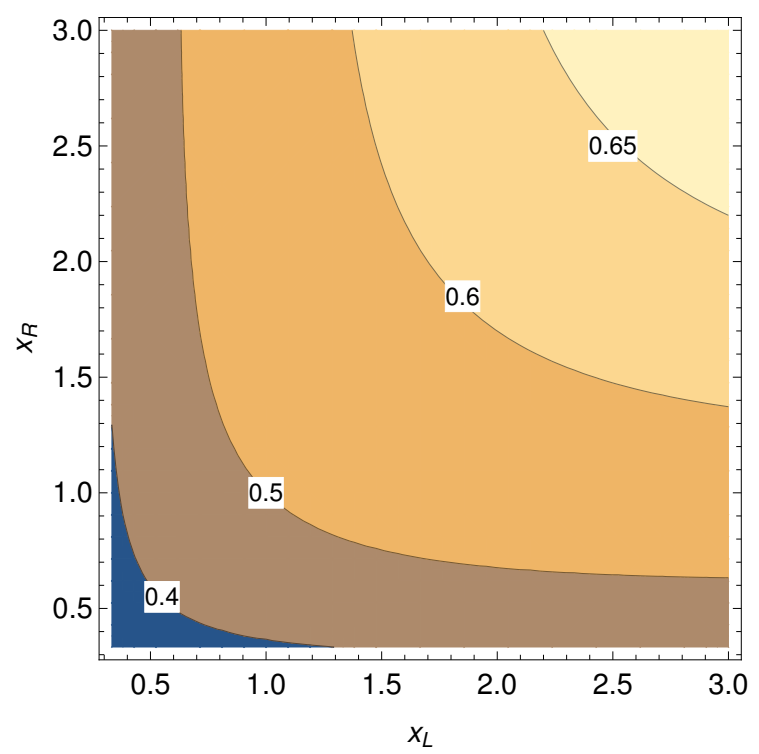

Figure 12. Dependence of function $g\left(x_{L}, x_{R}\right)$ of eq. (5.15) on the splitting between the slepton and bino masses.

The Higgs decays in supersymmetric extensions of the SM have already been studied e.g. in [84, 102-109]. In this section we analyse within the general MSSM the decays of the lighter CP-even Higgs boson $h$. The mass eigenstates formulae for the MSSM contributions to the effective leptonic Yukawa couplings of $h$ are given in eqs. (2.14)-(2.16) while the relevant MI expressions are collected in appendix E.3. The potentially largest contributions to $h \rightarrow \ell \ell^{\prime}$ decays come from the effects non decoupling in the limit of large SUSY masses and proportional to non-holomorphic trilinear terms (see eq. (E.22)). Assuming that flavour violating $A_{l}^{\prime I J}$ terms are the only source of LFV and using eqs. (3.4), (E.22) and (A.8), one can write

$$
B r\left(h \rightarrow \ell^{I} \bar{\ell}^{J}\right) \approx \frac{e^{4} M_{h}}{8192 \pi^{5} c_{W}^{4} \Gamma_{h}} \frac{\cos ^{2}(\alpha-\beta)}{\cos ^{2} \beta}\left(g\left(x_{\tilde{e}_{L I}}, x_{\tilde{e}_{R J}}\right)^{2}\left|\Delta_{L R}^{\prime I J}\right|^{2}+g\left(x_{\tilde{e}_{L J}}, x_{\tilde{e}_{R I}}\right)^{2}\left|\Delta_{L R}^{\prime J I}\right|^{2}\right),
$$

where $\alpha, \beta$ are the mixing angles in the Higgs sector (see appendix A), the dimensionless mass ratios are

$$
x_{\tilde{e}_{L(R) I}}=\frac{m_{\tilde{e}_{L(R) I}}}{\left|M_{1}\right|},
$$

and we defined

$$
g(x, y)=-\sqrt{x y} C_{0}(x, y, 1) .
$$

As can be seen from of figure 12, for reasonable mass splittings $g(x, y) \sim \mathcal{O}(1)$ and, inserting the numerical values of known quantities, one has

$$
\operatorname{Br}\left(h \rightarrow \ell^{I} \bar{\ell}^{J}\right) \sim 2 \cdot 10^{-4} \frac{\cos ^{2}(\alpha-\beta)}{\cos ^{2} \beta}\left|\Delta_{L R}^{\prime I J(J I)}\right|^{2} .
$$

Even if for large SUSY mass scale $\Delta_{L R}^{\prime I J}$ insertions are not constrained experimentally by other LFV measurements, $\operatorname{Br}\left(h \rightarrow \ell^{I} \bar{\ell}^{J}\right)$ cannot be arbitrarily large in the MSSM because 
$\Delta_{L R}^{\prime I J}$ are constrained to $\mathcal{O}(1)$ by the vacuum stability conditions and the requirement of the absence of charge and colour breaking (CCB) minima of the scalar potential (see e.g. discussion in [110]).

The Higgs mixing angle $\alpha$ is subject to strong radiative corrections from the squark sector and thus from the point of view of pure leptonic sector can be treated as a free parameter. However, the allowed values of the Higgs mixing angles $\alpha, \beta$ are limited by the existing experimental constraints (see e.g. figure 6 in appendix B of ref. [107]), thus also the overall pre-factor $\frac{\cos ^{2}(\alpha-\beta)}{\cos ^{2} \beta}$ in eq. (5.16) can be at most $\mathcal{O}(1)$. Summarising, the maximal $\operatorname{Br}\left(h \rightarrow \ell^{I} \bar{\ell}^{J}\right)$ which can be generated with the non-holomorphic trilinear terms is $\mathcal{O}\left(10^{-4}\right)$, not much below the current experimental sensitivities collected in table 2 (including decoupling contributions does not change this conclusion even for a light SUSY spectrum $[106,107])$. Further searches may therefore find the effects of non-holomorphic trilinear terms or provide stricter bounds on them.

Similar analysis could be done for non-decoupling contributions proportional to $\Delta_{L L}^{I J}$ and $\Delta_{R R}^{I J}$ parameters. However, in this case non-decoupling terms are proportional also either to the diagonal $A_{l}^{\prime}$ soft terms or to lepton Yukawa couplings, so the formulae become complicated and a more involved numerical analysis is required. Terms proportional to $\Delta_{L L}^{I J}$ and $\Delta_{R R}^{I J}$ multiplied by diagonal $A_{l}^{\prime}$ terms can generate similar LFV Higgs decay rates as the flavour off-diagonal non-holomorphic $A_{l}^{\prime}$-terms. However, assuming that all non-holomorphic terms vanish, and including only the Yukawa suppressed contributions one has a much stricter bound $\operatorname{Br}\left(h \rightarrow \ell^{I} \bar{\ell}^{J}\right) \lesssim 10^{-4}\left(Y_{l}^{I}\right)^{2}$ in the MSSM.

For a complete phenomenological analysis of LFV Higgs decays in the MSSM one would need to go beyond the one-loop analysis of this article. First, one would need to perform the matching of the MSSM on the 2HDM with generic Yukawa couplings including the resummation of the higher order chirally enhanced effects (see for example [54-56]). Then, one has to calculate the loop effects for flavour observables within this generic 2HDM [111].

\section{Conclusions}

New precision data in the lepton flavour sector are expected to come in the foreseeable future. The search for beyond the SM effects will require precision and efficient calculations in various BSM models. In this article lepton flavour violating processes within MSSM have been calculated using the Flavour Expansion Theorem, a recently developed new technique of a purely algebraic mass-insertion expansion of the amplitudes [31]. Both flavour-violating off-diagonal terms and flavour-conserving mass-insertions are considered. The expansion in the flavour conserving off-diagonal mass terms leads to a transparent qualitative understanding of the coefficients in front of the flavour violating mass insertions (see eq. (4.2)) in various decoupling limits. Most flavour violating one-loop amplitudes decouple as $v^{2} / M^{2}$ where $M$ is one of the soft SUSY breaking mass parameters. The exception are the Higgs flavour violating decays where the amplitudes decouple as $v^{2} / M_{A}^{2}$. We find that our full MI approximation, both in flavour violating and flavour conserving off-diagonal mass terms is an excellent approximation to the calculations in the mass eigenstates basis for a very broad pattern of supersymmetric spectra, in particular for highly non-degenerate spectra. This is 
useful because in the MI approximation we work directly with the Lagrangian parameters and can constrain them with experimental limits.

On the physics side, the considered processes are: $\ell \rightarrow \ell^{\prime} \gamma, \ell \rightarrow 3 \ell^{\prime}, \ell \rightarrow 2 \ell^{\prime} \ell^{\prime \prime}, h \rightarrow \ell \ell^{\prime}$ as well as $\mu \rightarrow e$ conversion in nuclei. The bounds on the flavour changing parameters of the MSSM have been updated and their sensitivity to the forthcoming experimental results in different channels has been discussed. We have emphasised that, given the foreseen experimental progress, precision measurements of different processes have very different potential for the discovery of supersymmetric effects. The radiative and leptonic muon decays are likely to remain the most important source of information on supersymmetric LFV. The leptonic decays play a complementary role to the radiative ones in eliminating some "blind spots" of weakly constrained by the latter LFV mass insertions. This is illustrated in sections 5.2 and 5.3. Our complete analytical MI expansion facilitates the investigation of the LFV processes when the SUSY spectra are non-degenerate and finding such "blind spots" with suppressed branching ratios and regions of correlations between various processes. This is illustrated in sections 5.2 and 5.3. The LFV Higgs decays are discussed in some detail. For the supersymmetric spectrum of order of $1 \mathrm{TeV}$, the current experimental limits on the LFV Higgs decays give several orders of magnitude weaker bounds on lepton violating $\mathrm{MI}$ than the radiative lepton decays. However, for the superpartner masses of several TeV Higgs decays provide stronger bounds than the latter because the bounds from Higgs decays do not scale with superpartner masses. We have also analysed the role of the so-called non-holomorphic $A$-terms in the flavour-violating Higgs boson decays, which can give branching ratios not much below the present experimental sensitivity.

\section{Acknowledgments}

ZF, WM, SP and JR are supported in part by the National Science Centre, Poland, under research grants DEC-2015/19/B/ST2/02848, DEC-2015/18/M/ST2/00054 and DEC2014/15/B/ST2/02157. U.N. is supported by BMBF under grant no. 05H15VKKB1. The work of A.C. is supported by an Ambizione Grant of the Swiss National Science Foundation (PZ00P2_154834). JR would also like to thank CERN for hospitality during his visits there.

\section{A MSSM Lagrangian and vertices}

Throughout this article we use the notation of refs. $[43,44]$ which is very similar to SLHA2 conventions [45], up to minor differences listed in table 6 .

For completeness, we collect here the definitions of the mass and mixing matrices for the supersymmetric particles and the relevant MSSM Feynman rules. The slepton and sneutrino mass and mixing matrices are defined as:

$$
\begin{gathered}
Z_{\nu}^{\dagger}\left(M_{L L}^{2}+\frac{M_{Z}^{2} \cos 2 \beta}{2} \hat{1}\right) Z_{\nu}=\operatorname{diag}\left(m_{\nu_{1}}^{2} \ldots m_{\nu_{3}}^{2}\right), \\
Z_{L}^{\dagger}\left(\begin{array}{l}
\left(\mathcal{M}_{L}^{2}\right)_{L L}\left(\mathcal{M}_{L}^{2}\right)_{L R} \\
\left(\mathcal{M}_{L}^{2}\right)_{L R}^{\dagger}\left(\mathcal{M}_{L}^{2}\right)_{R R}
\end{array}\right) Z_{L}=\operatorname{diag}\left(m_{L_{1}}^{2} \ldots m_{L_{6}}^{2}\right),
\end{gathered}
$$




\begin{tabular}{|c|c|}
\hline SLHA2 [45] & refs. [43,44] \\
\hline$\hat{T}_{U}, \hat{T}_{D}, \hat{T}_{E}$ & $-A_{u}^{T},+A_{d}^{T},+A_{l}^{T}$ \\
$\hat{m}_{\tilde{Q}}^{2}, \hat{m}_{\tilde{L}}^{2}$ & $m_{Q}^{2}, m_{L}^{2}$ \\
$\hat{m}_{\tilde{u}}^{2}, \hat{m}_{\tilde{d}}^{2}, \hat{m}_{\tilde{l}}^{2}$ & $\left(m_{U}^{2}\right)^{T},\left(m_{D}^{2}\right)^{T},\left(m_{E}^{2}\right)^{T}$ \\
$\mathcal{M}_{\tilde{u}}^{2}, \mathcal{M}_{\tilde{d}}^{2}$ & $\left(\mathcal{M}_{U}^{2}\right)^{T},\left(\mathcal{M}_{D}^{2}\right)^{T}$ \\
\hline
\end{tabular}

Table 6. Comparison of SLHA2 [45] and refs. [43, 44] conventions.

$$
\begin{aligned}
& \left(\mathcal{M}_{L}^{2}\right)_{L L}=\left(M_{L L}^{2}\right)^{T}+\frac{M_{Z}^{2} \cos 2 \beta}{2}\left(1-2 c_{W}^{2}\right) \hat{1}+\frac{v_{1}^{2} Y_{l}^{2}}{2} \\
& \left(\mathcal{M}_{L}^{2}\right)_{R R}=M_{R R}^{2}-\frac{M_{Z}^{2} \cos 2 \beta}{2} s_{W}^{2} \hat{1}+\frac{v_{1}^{2} Y_{l}^{2}}{2} \\
& \left(\mathcal{M}_{L}^{2}\right)_{L R}=\frac{1}{\sqrt{2}}\left(v_{2}\left(Y_{l} \mu^{\star}-A_{l}^{\prime}\right)+v_{1} A_{l}\right)
\end{aligned}
$$

where, as usual, we use $\tan \beta=v_{2} / v_{1}$ and $M_{L L}^{2}, M_{R R}^{2}, A_{l}, A_{l}^{\prime}, Y_{l}=-\sqrt{2} m_{l} / v_{1}$ are $3 \times 3$ matrices in flavour space.

The neutralino and chargino mass and mixing matrices can be written down as:

$$
\begin{gathered}
Z_{N}^{T}\left(\begin{array}{cccc}
M_{1} & 0 & -\frac{e v_{1}}{2 c_{W}} & \frac{e v_{2}}{2 c_{W}} \\
0 & M_{2} & \frac{e v_{1}}{2 s_{W}} & -\frac{e v_{2}}{2 s_{W}} \\
-\frac{e v_{1}}{2 c_{W}} & \frac{e v_{1}}{2 s_{W}} & 0 & -\mu \\
\frac{e v_{2}}{2 c_{W}} & -\frac{e v_{2}}{2 s_{W}} & -\mu & 0
\end{array}\right) Z_{N}=\operatorname{diag}\left(m_{\chi_{1}^{0}} \ldots m_{\chi_{4}^{0}}\right) \\
\left(Z_{-}\right)^{T}\left(\begin{array}{cc}
M_{2} & \frac{e v_{2}}{\sqrt{2} s_{W}} \\
\frac{e v_{1}}{\sqrt{2} s_{W}} & \mu
\end{array}\right) Z_{+}=\operatorname{diag}\left(m_{\chi_{1}}, m_{\chi_{2}}\right)
\end{gathered}
$$

We also use the following abbreviation for the matrix $Z_{R}$ parametrizing the mixing in the CP-even Higgs sector:

$$
Z_{R}=\left(\begin{array}{cc}
\cos \alpha & -\sin \alpha \\
\sin \alpha & \cos \alpha
\end{array}\right) .
$$

Below we list the vertices used in calculations of the LFV processes expressed in terms of the mixing matrices defined above.

1) Lepton-slepton-neutralino and lepton-sneutrino-chargino vertices (for an incoming charged lepton of flavour $I$ ):

$$
\begin{aligned}
V_{\ell \tilde{L} N, L}^{I i j} & =\frac{e}{\sqrt{2} s_{W} c_{W}} Z_{L}^{I i}\left(Z_{N}^{1 j} s_{W}+Z_{N}^{2 j} c_{W}\right)+Y_{l}^{I} Z_{L}^{(I+3) i} Z_{N}^{3 j}, \\
V_{\ell \tilde{L} N, R}^{I i j} & =\frac{-e \sqrt{2}}{c_{W}} Z_{L}^{(I+3) i} Z_{N}^{1 j \star}+Y_{l}^{I} Z_{L}^{I i} Z_{N}^{3 j \star}, \\
V_{\ell \tilde{\nu} C, L}^{I K j} & =-\frac{e}{s_{W}} Z_{+}^{1 j}, Z_{\nu}^{I K \star} \\
V_{\ell \tilde{\nu} C, R}^{I K j} & =-Y_{l}^{I} Z_{-}^{2 j \star} Z_{\nu}^{I K \star} .
\end{aligned}
$$


2) $Z$-chargino and $Z$-neutralino vertices:

$$
\begin{aligned}
V_{C C Z, L}^{i j} & =-\frac{e}{2 s_{W} c_{W}}\left(Z_{+}^{1 i *} Z_{+}^{1 j}+\delta^{i j}\left(c_{W}^{2}-s_{W}^{2}\right)\right), \\
V_{C C Z, R}^{i j} & =-\frac{e}{2 s_{W} c_{W}}\left(Z_{-}^{1 i} Z_{-}^{1 j *}+\delta^{i j}\left(c_{W}^{2}-s_{W}^{2}\right)\right), \\
V_{N N Z, L}^{i j} & =-V_{N N Z, R}^{j i}=\frac{e}{2 s_{W} c_{W}}\left(Z_{N}^{4 i *} Z_{N}^{4 j}-Z_{N}^{3 i *} Z_{N}^{3 j}\right) .
\end{aligned}
$$

3) CP-even-Higgs-slepton and CP-even-Higgs-sneutrino vertices:

$$
\begin{aligned}
V_{H L L}^{K i l}= & \sum_{C=1}^{3}\left(\frac{e^{2}}{2 c_{W}^{2}}\left(v_{1} Z_{R}^{1 K}-v_{2} Z_{R}^{2 K}\right)\left(\delta^{i l}+\frac{1-4 s_{W}^{2}}{2 s_{W}^{2}} Z_{L}^{C i \star} Z_{L}^{C l}\right)\right. \\
& -\left(Y_{l}^{C}\right)^{2} v_{1} Z_{R}^{1 K}\left(Z_{L}^{C i \star} Z_{L}^{C l}+Z_{L}^{(C+3) i \star} Z_{L}^{(C+3) l}\right) \\
& \left.-\frac{Z_{R}^{2 K}}{\sqrt{2}}\left(Y_{l}^{C *} \mu^{*} Z_{L}^{C i \star} Z_{L}^{(C+3) l}+Y_{l}^{C} \mu Z_{L}^{C l} Z_{L}^{(C+3) i \star}\right)\right) \\
& -\frac{1}{\sqrt{2}} \sum_{C, D=1}^{3}\left(Z_{R}^{1 K}\left(A_{l}^{C D \star} Z_{L}^{C l} Z_{L}^{(D+3) i \star}+A_{l}^{C D} Z_{L}^{C i \star} Z_{L}^{(D+3) l}\right)\right. \\
& \left.-Z_{R}^{2 K}\left(A_{l}^{\prime C D \star} Z_{L}^{C l} Z_{L}^{(D+3) i \star}+A_{l}^{C} C D Z_{L}^{C i \star} Z_{L}^{(D+3) l}\right)\right), \\
V_{H \tilde{\nu} \tilde{\nu}}^{K L I}= & -\frac{e^{2}}{4 s_{W}^{2} c_{W}^{2}}\left(v_{1} Z_{R}^{1 K}-v_{2} Z_{R}^{2 K}\right) \delta_{L I} .
\end{aligned}
$$

4) CP-odd-Higgs-slepton and CP-odd-Higgs-sneutrino vertices:

$$
\begin{aligned}
V_{A L L}^{1 i l}= & \frac{i \cos \beta}{\sqrt{2}} \sum_{C, D=1}^{3}\left(\left(A_{l}^{C D *} \tan \beta+A_{l}^{\prime C D *}-Y_{l}^{C} \mu \delta^{C D}\right) Z_{L}^{C j} Z_{L}^{(D+3) i \star}\right. \\
& \left.-\left(A_{l}^{C D} \tan \beta+A_{l}^{\prime C D}-Y_{l}^{C} \mu^{*} \delta^{C D}\right) Z_{L}^{C i \star} Z_{L}^{(D+3) j}\right), \\
V_{A \tilde{\nu} \tilde{\nu}}^{1 L I}= & 0 .
\end{aligned}
$$

5) CP-even-Higgs-neutralino and CP-even-Higgs-chargino vertices:

$$
\begin{aligned}
V_{N H N, L}^{i K l}= & V_{N H N, R}^{i K l *}=\frac{e}{2 s_{W} c_{W}}\left(\left(Z_{R}^{1 K} Z_{N}^{3 l}-Z_{R}^{2 K} Z_{N}^{4 l}\right)\left(Z_{N}^{1 i} s_{W}-Z_{N}^{2 i} c_{W}\right)\right. \\
& \left.+\left(Z_{R}^{1 K} Z_{N}^{3 i}-Z_{R}^{2 K} Z_{N}^{4 i}\right)\left(Z_{N}^{1 l} s_{W}-Z_{N}^{2 l} c_{W}\right)\right), \\
V_{C H C, L}^{i K l}= & V_{C H C, R}^{i K l *}=-\frac{e}{\sqrt{2} s_{W}}\left(Z_{R}^{1 K} Z_{-}^{2 i} Z_{+}^{1 l}+Z_{R}^{2 K} Z_{-}^{1 i} Z_{+}^{2 l}\right) .
\end{aligned}
$$

6) CP-odd-Higgs-neutralino and CP-odd-Higgs-chargino vertices:

$$
\begin{aligned}
V_{N A N, L}^{i 1 l}= & V_{N A N, R}^{i 1 l *}=\frac{-i e^{2}}{4 s_{W}^{2} c_{W}^{2} M_{Z}}\left(\left(v_{2} Z_{N}^{3 j}-v_{1} Z_{N}^{4 j}\right)\left(Z_{N}^{1 i} s_{W}-Z_{N}^{2 i} c_{W}\right)\right. \\
& \left.+\left(v_{2} Z_{N}^{3 i}-v_{1} Z_{N}^{4 i}\right)\left(Z_{N}^{1 j} s_{W}-Z_{N}^{2 j} c_{W}\right)\right) \\
V_{C A C, L}^{i 1 l}= & V_{C A C, R}^{i 1 l *}=\frac{i e^{2}}{2 \sqrt{2} s_{W}^{2} M_{W}}\left(v_{2} Z_{-}^{2 i} Z_{+}^{1 j}+v_{1} Z_{-}^{1 i} Z_{+}^{2 j}\right) .
\end{aligned}
$$


7) $Z$-slepton vertex:

$$
V_{L L Z}^{i j}=\frac{e}{2 s_{W} c_{W}}\left(Z_{L}^{k i *} Z_{L}^{k j}-2 s_{W}^{2} \delta^{i j}\right)
$$

\section{B Loop integrals}

We define the following loop integrals for 2-point and 3-point functions with non-vanishing external momenta $p$ and $q$ :

$$
\begin{aligned}
\frac{i}{(4 \pi)^{2}} B_{0}\left(p, m_{1}, m_{2}\right) & =\int \frac{d^{4} k}{(2 \pi)^{4}} \frac{1}{\left(k^{2}-m_{1}^{2}\right)\left((k-p)^{2}-m_{2}^{2}\right)}, \\
\frac{i}{(4 \pi)^{2}} p_{\mu} B_{1}\left(p, m_{1}, m_{2}\right) & =\int \frac{d^{4} k}{(2 \pi)^{4}} \frac{k_{\mu}}{\left(k^{2}-m_{1}^{2}\right)\left((k-p)^{2}-m_{2}^{2}\right)}, \\
\frac{i}{(4 \pi)^{2}} C_{2 n}\left(p, q, m_{1}, m_{2}, m_{3}\right) & =\int \frac{d^{4} k}{(2 \pi)^{4}} \frac{\left(k^{2}\right)^{n}}{\left(k^{2}-m_{1}^{2}\right)\left((k+p)^{2}-m_{2}^{2}\right)\left((k+p+q)^{2}-m_{3}^{2}\right)}, \\
\frac{i}{(4 \pi)^{2}}\left(p_{\mu} C_{11}\left(p, q, m_{1}, m_{2}, m_{3}\right)\right. & \left.+q_{\mu} C_{12}\left(p, q, m_{1}, m_{2}, m_{3}\right)\right) \\
& =\int \frac{d^{4} k}{(2 \pi)^{4}} \frac{k_{\mu}}{\left(k^{2}-m_{1}^{2}\right)\left((k+p)^{2}-m_{2}^{2}\right)\left((k+p+q)^{2}-m_{3}^{2}\right)} .
\end{aligned}
$$

In our expanded results we need only the integrals above, their derivatives and higher point 1-loop integrals calculated at vanishing external momenta. Let us define

$$
\frac{i}{(4 \pi)^{2}} L_{i}^{2 n}\left(m_{1}, \ldots, m_{i}\right)=\int \frac{d^{4} k}{(2 \pi)^{4}} \frac{\left(k^{2}\right)^{n}}{\prod_{j=1}^{i}\left(k^{2}-m_{j}^{2}\right)} .
$$

In common notation $L_{3}^{2 n}=C_{2 n}, L_{4}^{2 n}=D_{2 n}, L_{5}^{2 n}=E_{2 n}$ etc.

For $i \geq 3$ one has:

$$
\begin{aligned}
& L_{i}^{0}\left(m_{1}, \ldots, m_{i}\right)=-\sum_{j=2}^{i} \frac{m_{j}^{2} \log \frac{m_{j}^{2}}{m_{1}^{2}}}{\prod_{k=1, k \neq j}^{i}\left(m_{j}^{2}-m_{k}^{2}\right)}, \\
& L_{i}^{2}\left(m_{1}, \ldots, m_{i}\right)=\sum_{j=2}^{i} \frac{m_{j}^{4} \log \frac{m_{j}^{2}}{m_{1}^{2}}}{\prod_{k=1, k \neq j}^{i}\left(m_{j}^{2}-m_{k}^{2}\right)},
\end{aligned}
$$

(with the exception of $L_{3}^{2} \equiv C_{2}$ having also an infinite part, which however is always cancelled out in flavour violating processes and is thus not given here explicitly).

To simplify our formulae, we use the relation

$$
\begin{aligned}
2 L_{i}^{0}\left(m_{1}, m_{2}, \ldots, m_{i}\right)= & L_{i+1}^{2}\left(m_{1}, m_{1}, m_{2}, \ldots, m_{i}\right)+L_{i+1}^{2}\left(m_{1}, m_{2}, m_{2}, \ldots, m_{i}\right) \\
& +\ldots+L_{i+1}^{2}\left(m_{1}, \ldots, m_{i-1}, m_{i}, m_{i}\right),
\end{aligned}
$$

which can be obtained by differentiating with respect to $\lambda$ the integral form of the homogeneity property

$$
L_{i}^{0}\left(\lambda m_{1}, \ldots, \lambda m_{i}\right)=\lambda^{4-2 i} L_{i}^{0}\left(m_{1}, \ldots, m_{i}\right)
$$


and using the relation $(k=1, \ldots, i)$

$$
\begin{aligned}
m_{k}^{2} L_{i+1}^{0}\left(m_{1}, \ldots, m_{k}, m_{k}, \ldots, m_{i}\right)= & L_{i+1}^{2}\left(m_{1}, \ldots, m_{k}, m_{k}, \ldots, m_{i}\right) \\
& -L_{i}^{0}\left(m_{1}, \ldots, m_{k}, \ldots, m_{i}\right) .
\end{aligned}
$$

In addition, we define the following integrals:

$$
\begin{aligned}
C_{0}^{\prime}\left(m_{1}, m_{2}, m_{3}\right)= & \left.\frac{\partial C_{0}\left(p, q, m_{1}, m_{2}, m_{3}\right)}{\partial q^{2}}\right|_{p=q=0} \\
= & \frac{2 m_{2}^{2} m_{3}^{2}-m_{1}^{2}\left(m_{2}^{2}+m_{3}^{2}\right)}{2\left(m_{1}^{2}-m_{2}^{2}\right)\left(m_{1}^{2}-m_{3}^{2}\right)\left(m_{2}^{2}-m_{3}^{2}\right)^{2}} \\
& +\frac{m_{1}^{4} \log \frac{m_{1}^{2}}{m_{2}^{2}}}{2\left(m_{1}^{2}-m_{2}^{2}\right)^{2}\left(m_{1}^{2}-m_{3}^{2}\right)^{2}}+\frac{m_{3}^{2}\left(m_{3}^{4}-2 m_{1}^{2} m_{2}^{2}+m_{2}^{2} m_{3}^{2}\right) \log \frac{m_{3}^{2}}{m_{2}^{2}}}{2\left(m_{1}^{2}-m_{3}^{2}\right)^{2}\left(m_{2}^{2}-m_{3}^{2}\right)^{3}} \\
C_{11}\left(m_{1}, m_{2}\right)= & -\frac{m_{1}^{2}-3 m_{2}^{2}}{4\left(m_{1}^{2}-m_{2}^{2}\right)^{2}}+\frac{m_{2}^{4}}{2\left(m_{1}^{2}-m_{2}^{2}\right)^{3}} \log \frac{m_{2}^{2}}{m_{1}^{2}} \\
C_{12}\left(m_{1}, m_{2}\right)= & -\frac{m_{1}^{2}+m_{2}^{2}}{2\left(m_{1}^{2}-m_{2}^{2}\right)^{2}}-\frac{m_{1}^{2} m_{2}^{2}}{\left(m_{1}^{2}-m_{2}^{2}\right)^{3}} \log \frac{m_{2}^{2}}{m_{1}^{2}} \\
C_{23}\left(m_{1}, m_{2}\right)= & -\frac{m_{1}^{4}-5 m_{1}^{2} m_{2}^{2}-2 m_{2}^{4}}{12\left(m_{1}^{2}-m_{2}^{2}\right)^{3}}+\frac{m_{1}^{2} m_{2}^{4}}{2\left(m_{1}^{2}-m_{2}^{2}\right)^{4}} \log \frac{m_{2}^{2}}{m_{1}^{2}} \\
C_{01}\left(m_{1}, m_{2}\right)= & \frac{7 m_{1}^{4}-29 m_{1}^{2} m_{2}^{2}+16 m_{2}^{4}}{36\left(m_{1}^{2}-m_{2}^{2}\right)^{3}}+\frac{m_{2}^{4}\left(-3 m_{1}^{2}+2 m_{2}^{2}\right)}{6\left(m_{1}^{2}-m_{2}^{2}\right)^{4}} \log \frac{m_{2}^{2}}{m_{1}^{2}} \\
C_{02}\left(m_{1}, m_{2}\right)= & \frac{11 m_{1}^{4}-7 m_{1}^{2} m_{2}^{2}+2 m_{2}^{4}}{36\left(m_{1}^{2}-m_{2}^{2}\right)^{3}}+\frac{m_{1}^{6}}{6\left(m_{1}^{2}-m_{2}^{2}\right)^{4}} \log \frac{m_{2}^{2}}{m_{1}^{2}}
\end{aligned}
$$

\section{Divided differences}

The expansion of the amplitudes given in the mass eigenbasis in terms of mass insertions can be naturally expressed [31] by the so-called divided differences of the loop functions.

In case of a function of a single argument, $f(x)$, divided differences are defined recursively as:

$$
\begin{aligned}
f^{[0]}(x) & =f(x), \\
f^{[1]}(x, y) & =\frac{f^{[0]}(x)-f^{[0]}(y)}{x-y}, \\
f^{[2]}(x, y, z) & =\frac{f^{[1]}(x, y)-f^{[1]}(x, z)}{y-z}, \\
& \ldots
\end{aligned}
$$

As can be easily checked, a divided difference of order $n$ is symmetric under permutation of any subset of its arguments. It also has a smooth limit for degenerate arguments:

$$
\lim _{\left\{x_{0}, \ldots, x_{m}\right\} \rightarrow\{\xi, \ldots, \xi\}} f^{[k]}\left(x_{0}, \ldots, x_{k}\right)=\frac{1}{m !} \frac{\partial^{m}}{\partial \xi^{m}} f^{[k-m]}\left(\xi, x_{m+1} \ldots, x_{k}\right) .
$$


To compactify the formulae for functions of many arguments, we use the notation

$$
f^{[k]}\left(x_{0}, \ldots, x_{k}\right) \equiv f\left(\left\{x_{0}, \ldots, x_{k}\right\}\right),
$$

where the order of the divided difference is defined by the number of arguments inside curly brackets. Then, for example a divided difference of the 1st order in the 1st argument and of the 3 rd order in the 2nd argument for the function of 3 variables, $g(x, y, z)$, can be written down as:

$$
g\left(\left\{x_{1}, x_{2}\right\},\left\{y_{1}, y_{2}, y_{3}, y_{4}\right\}, z\right) .
$$

For the loop functions defined in appendix B one should note that their natural arguments are squares of masses. However, we use $m_{i}$ 's instead of $m_{i}^{2}$ 's to compactify the notation. Thus, for loop functions we write divided differences as

$$
L\left(m_{1}, \ldots,\left\{m_{i}, m_{i}^{\prime}\right\}, \ldots, m_{n}\right)=\frac{L\left(m_{1}, \ldots, m_{i}, \ldots, m_{n}\right)-L\left(m_{1}, \ldots, m_{i}^{\prime}, \ldots, m_{n}\right)}{m_{i}^{2}-m_{i}^{\prime 2}},
$$

with squared masses in the denominator.

The FET expansion works for any transition amplitude, also in the case of nonvanishing external momenta or for multi-loop calculations. However, it is particularly effective for 1-loop functions with vanishing external momenta, due to the fact that the notion of the divided differences is naturally encoded in the structure of such functions: a divided difference of a $n$-point scalar 1-loop function is a $(n+1)$-point function (see eq. 3.13 in ref. [31] for generalisation to the case of non-vanishing external momenta). Thus, for example one has

$$
\begin{aligned}
B_{0}\left(m_{1},\left\{m_{2}, m_{3}\right\}\right) & =B_{0}\left(\left\{m_{1}, m_{2}\right\}, m_{3}\right)=C_{0}\left(m_{1}, m_{2}, m_{3}\right) \\
B_{0}\left(m_{1},\left\{m_{2}, m_{3}, m_{4}\right\}\right) & =C_{0}\left(m_{1}, m_{2},\left\{m_{3}, m_{4}\right\}\right)=D_{0}\left(m_{1}, m_{2}, m_{3}, m_{4}\right)
\end{aligned}
$$

We use such relations extensively to find cancellations between various terms and to identify the lowest non-vanishing order of mass insertion expansion for a given process.

\section{Box diagrams in the mass eigenstates basis}

There are four types of box diagrams with four external leptons involving slepton (sneutrinos) and neutralinos (charginos) in the loop, displayed in figure 13. Both chargino-sneutrino and neutralino-slepton pairs contribute to diagrams A) and B), while only neutralinos (Majorana fermions) can be exchanged in the "crossed" diagrams C) and D).

Using whenever necessary Fierz identities, the amplitudes describing each of the diagrams $N=A, B, C, D$ can be brought into the form

$$
i A_{N}^{J I K L}=i \sum_{Q=V, S, T} B_{N Q X Y}^{J I K L}\left[\bar{u}\left(p_{J}\right) \Gamma_{Q} P_{X} u\left(p_{I}\right)\right]\left[\bar{u}\left(p_{K}\right) \Gamma_{Q} P_{Y} v\left(p_{L}\right)\right]
$$

with $\Gamma_{V}=\gamma^{\mu}, \Gamma_{S}=1$ and $\Gamma_{T}=\sigma_{\mu \nu}$. Note that for $\Gamma_{T}$ only the case $X=Y$ is non vanishing. Assuming that the generic couplings for an incoming lepton $\ell^{I}$ - an incoming 


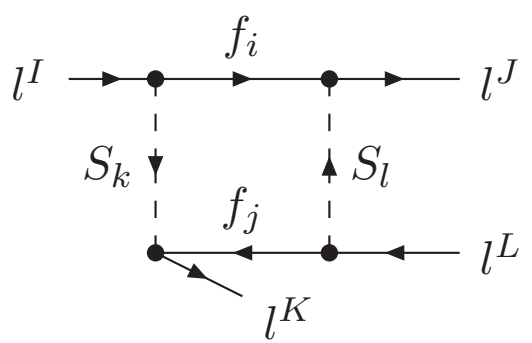

A)

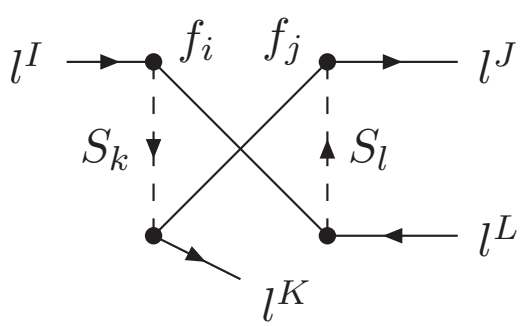

C)

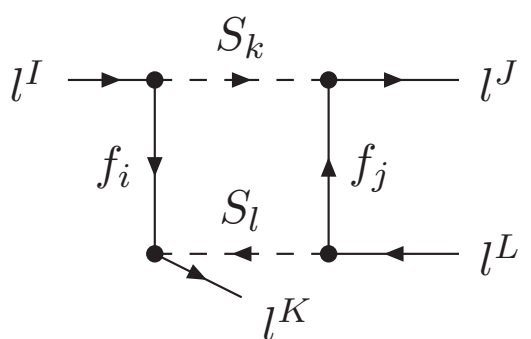

B)

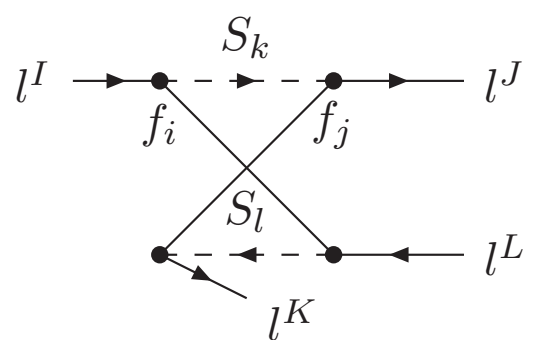

D)

Figure 13. MSSM box diagrams with 4 external charged leptons.

scalar particle $S_{k}$ and an outgoing fermion $f_{i}$ takes the form

$$
i V_{\ell S f}^{I k i}=i\left(A_{\ell S f}^{I k i} P_{L}+B_{\ell S f}^{I k i} P_{R}\right)
$$

the contribution from diagram A) in figure 13) to the Wilson coefficients $B_{Q X Y}$ can be written down as:

$$
\begin{aligned}
(4 \pi)^{2} B_{A V L L}^{J I K L} & =\frac{1}{4} A_{\ell S f}^{I k i} A_{\ell S f}^{J l i *} A_{\ell S f}^{K k j *} A_{\ell S f}^{L l j} D_{2}, \\
(4 \pi)^{2} B_{A V R R}^{J I K L} & =\frac{1}{4} B_{\ell S f}^{I k i} B_{\ell S f}^{J l i *} B_{\ell S f}^{K k j *} B_{\ell S f}^{L l j} D_{2}, \\
(4 \pi)^{2} B_{A V L R}^{J I K L} & =\frac{1}{4} A_{\ell S f}^{I k i} A_{\ell S f}^{J l i *} B_{\ell S f}^{K k j *} B_{\ell S f}^{L l j} D_{2}, \\
(4 \pi)^{2} B_{A V R L}^{J I K L} & =\frac{1}{4} B_{\ell S f}^{I k i} B_{\ell S f}^{J l i *} A_{\ell S f}^{K k j *} A_{\ell S f}^{L l j} D_{2}, \\
(4 \pi)^{2} B_{A S L L}^{J I K L} & =A_{\ell S f}^{I k i} B_{\ell S f}^{J l i *} B_{\ell S f}^{K k j *} A_{\ell S f}^{L l j} m_{f_{i}} m_{f_{j}} D_{0}, \\
(4 \pi)^{2} B_{A S R R}^{J I K L} & =B_{\ell S f}^{I k i} A_{\ell S f}^{J l i *} A_{\ell S f}^{K k j *} B_{\ell S f}^{L l j} m_{f_{i}} m_{f_{j}} D_{0}, \\
(4 \pi)^{2} B_{A S L R}^{J I K L} & =A_{\ell S f}^{I k i} B_{\ell S f}^{J l i *} A_{\ell S f}^{K k *} B_{\ell S f}^{L l j} m_{f_{i}} m_{f_{j}} D_{0}, \\
(4 \pi)^{2} B_{A S R L}^{J I K L} & =B_{\ell S f}^{I k i} A_{\ell S f}^{J l i *} B_{\ell S f}^{K k j *} A_{\ell S f}^{L l j} m_{f_{i}} m_{f_{j}} D_{0}, \\
(4 \pi)^{2} B_{A T L}^{J I K L} & =0, \\
(4 \pi)^{2} B_{A T R}^{J I K L} & =0,
\end{aligned}
$$

where $D_{0}, D_{2}$ above are the abbreviations for 4-point loop functions with respective mass arguments, $D_{0}=D_{0}\left(m_{f_{i}}, m_{f_{j}}, m_{S_{k}}, m_{S_{l}}\right), D_{2}=D_{2}\left(m_{f_{i}}, m_{f_{j}}, m_{S_{k}}, m_{S_{l}}\right)$ (see appendix B). 
Using the same notation, the contributions from diagram B), C), D) are:

$$
\begin{aligned}
& (4 \pi)^{2} B_{B V L L}^{J I K L}=\frac{1}{4} A_{\ell S f}^{I k i} A_{\ell S f}^{J k j *} A_{\ell S f}^{K l i *} A_{\ell S f}^{L l j} D_{2}, \\
& (4 \pi)^{2} B_{B V R R}^{J I K L}=\frac{1}{4} B_{\ell S f}^{I k i} B_{\ell S f}^{J k j *} B_{\ell S f}^{K l i *} B_{\ell S f}^{L l j} D_{2}, \\
& (4 \pi)^{2} B_{B V L R}^{J I K L}=-\frac{1}{2} A_{\ell S f}^{I k i} A_{\ell S f}^{J k j *} B_{\ell S f}^{K l i *} B_{\ell S f}^{L l j} m_{f_{i}} m_{f_{j}} D_{0}, \\
& (4 \pi)^{2} B_{B V R L}^{J I K L}=-\frac{1}{2} B_{\ell S f}^{I k i} B_{\ell S f}^{J k j *} A_{\ell S f}^{K l i *} A_{\ell S f}^{L l j} m_{f_{i}} m_{f_{j}} D_{0}, \\
& (4 \pi)^{2} B_{B S L L}^{J I K L}=-\frac{1}{2} A_{\ell S f}^{I k i} B_{\ell S f}^{J k j *} B_{\ell S f}^{K l i *} A_{\ell S f}^{L l j} m_{f_{i}} m_{f_{j}} D_{0}, \\
& (4 \pi)^{2} B_{B S R R}^{J I K L}=-\frac{1}{2} B_{\ell S f}^{I k i} A_{\ell S f}^{J k j *} A_{\ell S f}^{K l i *} B_{\ell S f}^{L l j} m_{f_{i}} m_{f_{j}} D_{0}, \\
& (4 \pi)^{2} B_{B S L R}^{J I K L}=-\frac{1}{2} A_{\ell S f}^{I k i} B_{\ell S f}^{J k j *} A_{\ell S f}^{K l i *} B_{\ell S f}^{L l j} D_{2}, \\
& (4 \pi)^{2} B_{B S R L}^{J I K L}=-\frac{1}{2} B_{\ell S f}^{I k i} A_{\ell S f}^{J k j *} B_{\ell S f}^{K l i *} A_{\ell S f}^{L l j} D_{2}, \\
& (4 \pi)^{2} B_{B T L}^{J I K L}=-\frac{1}{8} A_{\ell S f}^{I k i} B_{\ell S f}^{J k j *} B_{\ell S f}^{K l i *} A_{\ell S f}^{L l j} m_{f_{i}} m_{f_{j}} D_{0}, \\
& (4 \pi)^{2} B_{B T R}^{J I K L}=-\frac{1}{8} B_{\ell S f}^{I k i} A_{\ell S f}^{J k j *} A_{\ell S f}^{K l i *} B_{\ell S f}^{L l j} m_{f_{i}} m_{f_{j}} D_{0}, \\
& (4 \pi)^{2} B_{C V L L}^{J I K L}=\frac{1}{2} A_{\ell S f}^{I k i} A_{\ell S f}^{L l i} A_{\ell S f}^{J l j *} A_{\ell S f}^{K k j *} m_{f_{i}} m_{f_{j}} D_{0}, \\
& (4 \pi)^{2} B_{C V R R}^{J I K L}=\frac{1}{2} B_{\ell S f}^{I k i} B_{\ell S f}^{L l i} B_{\ell S f}^{J l j *} B_{\ell S f}^{K k j *} m_{f_{i}} m_{f_{j}} D_{0}, \\
& (4 \pi)^{2} B_{C V L R}^{J I K L}=\frac{1}{4} B_{\ell S f}^{I k i} A_{\ell S f}^{L l i} B_{\ell S f}^{J l j *} A_{\ell S f}^{K k j *} D_{2}, \\
& (4 \pi)^{2} B_{C V R L}^{J I K L}=\frac{1}{4} A_{\ell S f}^{I k i} B_{\ell S f}^{L l i} A_{\ell S f}^{J l j *} B_{\ell S f}^{K k j *} D_{2}, \\
& (4 \pi)^{2} B_{C S L L}^{J I K L}=-\frac{1}{2} A_{\ell S f}^{I k i} A_{\ell S f}^{L l i} B_{\ell S f}^{J l j *} B_{\ell S f}^{K k j *} m_{f_{i}} m_{f_{j}} D_{0}, \\
& (4 \pi)^{2} B_{C S R R}^{J I K L}=-\frac{1}{2} B_{\ell S f}^{I k i} B_{\ell S f}^{L l i} A_{\ell S f}^{J l j *} A_{\ell S f}^{K k j *} m_{f_{i}} m_{f_{j}} D_{0}, \\
& (4 \pi)^{2} B_{C S L R}^{J I K L}=\frac{1}{2} B_{\ell S f}^{I k i} A_{\ell S f}^{L l i} A_{\ell S f}^{J l j *} B_{\ell S f}^{K k j *} D_{2}, \\
& (4 \pi)^{2} B_{C S R L}^{J I K L}=\frac{1}{2} A_{\ell S f}^{I k i} B_{\ell S f}^{L l i} B_{\ell S f}^{J l j *} A_{\ell S f}^{K k j *} D_{2}, \\
& (4 \pi)^{2} B_{C T L}^{J I K L}=\frac{1}{8} A_{\ell S f}^{I k i} A_{\ell S f}^{L l i} B_{\ell S f}^{J l j *} B_{\ell S f}^{K k j *} m_{f_{i}} m_{f_{j}} D_{0}, \\
& (4 \pi)^{2} B_{C T R}^{J I K L}=\frac{1}{8} B_{\ell S f}^{I k i} B_{\ell S f}^{L l i} A_{\ell S f}^{J l j *} A_{\ell S f}^{K k j *} m_{f_{i}} m_{f_{j}} D_{0}, \\
& (4 \pi)^{2} B_{D V L L}^{J I K L}=\frac{1}{2} A_{\ell S f}^{I k i} A_{\ell S f}^{L l i} A_{\ell S f}^{J k j *} A_{\ell S f}^{K l j *} m_{f_{i}} m_{f_{j}} D_{0}, \\
& (4 \pi)^{2} B_{D V R R}^{J I K L}=\frac{1}{2} B_{\ell S f}^{I k i} B_{\ell S f}^{L l i} B_{\ell S f}^{J k j *} B_{\ell S f}^{K l j *} m_{f_{i}} m_{f_{j}} D_{0}, \\
& (4 \pi)^{2} B_{D V L R}^{J I K L}=-\frac{1}{4} B_{\ell S f}^{I k i} A_{\ell S f}^{L l i} B_{\ell S f}^{J k j *} A_{\ell S f}^{K l j *} D_{2},
\end{aligned}
$$




$$
\begin{aligned}
(4 \pi)^{2} B_{D V R L}^{J I K L} & =-\frac{1}{4} A_{\ell S f}^{I k i} B_{\ell S f}^{L l i} A_{\ell S f}^{J k j *} B_{\ell S f}^{K l j *} D_{2}, \\
(4 \pi)^{2} B_{D S L L}^{J I K L} & =-\frac{1}{2} A_{\ell S f}^{I k i} A_{\ell S f}^{L l i} B_{\ell S f}^{J k j *} B_{\ell S f}^{K l j *} m_{f_{i}} m_{f_{j}} D_{0}, \\
(4 \pi)^{2} B_{D S R R}^{J I K L} & =-\frac{1}{2} B_{\ell S f}^{I k i} B_{\ell S f}^{L l i} A_{\ell S f}^{J k j *} A_{\ell S f}^{K l j *} m_{f_{i}} m_{f_{j}} D_{0}, \\
(4 \pi)^{2} B_{D S L R}^{J I K L} & =-\frac{1}{2} B_{\ell S f}^{I k i} A_{\ell S f}^{L l i} A_{\ell S f}^{J k j *} B_{\ell S f}^{K l j *} D_{2}, \\
(4 \pi)^{2} B_{D S R L}^{J I K L} & =-\frac{1}{2} A_{\ell S f}^{I k i} B_{\ell S f}^{L l i} B_{\ell S f}^{J k j *} A_{\ell S f}^{K l j *} D_{2}, \\
(4 \pi)^{2} B_{D T L}^{J I K L} & =\frac{1}{8} A_{\ell S f}^{I k i} A_{\ell S f}^{L l i} B_{\ell S f}^{J k j *} B_{\ell S f}^{K l j *} m_{f_{i}} m_{f_{j}} D_{0}, \\
(4 \pi)^{2} B_{D T R}^{J I K L} & =\frac{1}{8} B_{\ell S f}^{I k i} B_{\ell S f}^{L l i} A_{\ell S f}^{J k j *} A_{\ell S f}^{K l j *} m_{f_{i}} m_{f_{j}} D_{0} .
\end{aligned}
$$

To obtain the actual MSSM contributions to the 4-lepton operators, one should add terms from eqs. (D.3), (D.4) with replacements $f \rightarrow C, S \rightarrow \tilde{\nu}, A_{\ell S f} \rightarrow V_{\ell \tilde{\nu} C, L}, B_{\ell S f} \rightarrow$ $V_{\ell \tilde{\nu} C, R}$ and $f \rightarrow N, S \rightarrow \tilde{L}, A_{\ell S f} \rightarrow V_{\ell \tilde{L} N, L}, B_{\ell S f} \rightarrow V_{\ell \tilde{L} N, R}$ (summing over repeated indices of loop particles) and terms from eqs. (D.5), (D.6), substituting there only $f \rightarrow N, S \rightarrow$ $\tilde{L}, A_{\ell S f} \rightarrow V_{\ell \tilde{L} N, L}, B_{\ell S f} \rightarrow V_{\ell \tilde{L} N, R}$

The contributions to 2-quark 2-lepton operators can be obtained from diagrams A) and C) by replacing $\ell_{K}$ and $\ell_{L}$ with $q_{K}$ and $q_{L}$ as defined in eq. (2.28). Therefore, the expressions for $B_{q} Q X Y$ can be obtained replacing vertices of leptons $\ell^{K}$ and $\ell^{L}$ by the relevant quark-squark vertices. Such vertices are not listed in appendix A but can be found in refs. [43, 44]. The explicit form of $\ell \ell d d$ box amplitudes can be also found in appendix A.3 of ref. [112].

\section{E Effective lepton couplings in the leading MI order}

We list below the MI expanded expressions for the leptonic penguin and box diagram amplitudes. For penguins we follow the decomposition of eq. (4.2), with $F_{X Y}$ denoting functions of flavour diagonal SUSY parameters multiplying the respective slepton mass insertions:

$$
\begin{aligned}
F_{X}^{I J}=\frac{1}{(4 \pi)^{2}} & \left(F_{X L L}^{I J} \Delta_{L L}^{I J}+F_{X R R}^{I J} \Delta_{R R}^{J I}\right. \\
& \left.+F_{X}^{I J}{ }_{A L R} \Delta_{L R}^{J I}+F_{X B L R}^{I J} \Delta_{L R}^{I J *}+F_{X}^{\prime I J}{ }_{A L R} \Delta_{L R}^{\prime J I}+F_{X B L R}^{\prime J J} \Delta_{L R}^{\prime I J *}\right) .
\end{aligned}
$$

To compactify the notation, we also introduce the abbreviation

$$
\bar{M}_{X Y}^{I J}=\sqrt{\left(M_{X X}^{2}\right)^{I I}\left(M_{Y Y}^{2}\right)^{J J}}
$$

where $X, Y=L$ or $R$.

\section{E.1 Lepton-photon vertex}

\section{E.1.1 Tensor (magnetic) couplings}

After performing MI expansion, one can see that terms coming from $F_{\gamma A}$ in eq. (2.4) are always suppressed by the powers of lepton Yukawa couplings or lepton masses, and may 
add to or cancel terms generated from $F_{\gamma L B}, F_{\gamma R B}$. Thus, in the expressions below we give the sum of both types of contributions.

The chargino contributions contain only terms proportional to LL slepton mass insertions (see appendix $\mathrm{C}$ for the notation of divided differences and curly brackets around the function arguments)

$$
\begin{aligned}
\left(F_{\gamma L L}\right)_{C}^{J I}= & \frac{e^{2} v_{1} Y_{L}^{J}}{2 \sqrt{2} s_{W}^{2}} \bar{M}_{L L}^{I J}\left(C_{11}\left(\left|M_{2}\right|,\left\{m_{\tilde{\nu}_{I}}, m_{\tilde{\nu}_{J}}\right\}\right)\right. \\
& +C_{11}\left(|\mu|,\left\{m_{\tilde{\nu}_{I}}, m_{\tilde{\nu}_{J}}\right\}\right)-C_{23}\left(\left|M_{2}\right|,\left\{m_{\tilde{\nu}_{I}}, m_{\tilde{\nu}_{J}}\right\}\right) \\
& \left.+\left(|\mu|^{2}+\left|M_{2}\right|^{2}+2 \mu^{*} M_{2}^{*} \tan \beta\right) C_{11}\left(\left\{|\mu|,\left|M_{2}\right|\right\},\left\{m_{\tilde{\nu}_{I}}, m_{\tilde{\nu}_{J}}\right\}\right)\right)
\end{aligned}
$$

The non-vanishing neutralino contributions are:

$$
\begin{aligned}
\left(F_{\gamma} L L\right)_{N}^{J I}= & \frac{e^{2}}{2 c_{W}^{2}} \bar{M}_{L L}^{I J}\left(M_{1}^{\star} C_{12}\left(\left\{m_{\tilde{e}_{L I}}, m_{\tilde{e}_{L J}}, m_{\tilde{e}_{R J}}\right\},\left|M_{1}\right|\right)\left(M_{L R}^{2}\right)_{J J}\right. \\
& -\frac{v_{1}}{2 \sqrt{2}} Y_{L}^{J}\left(\frac{c_{W}^{2}}{s_{W}^{2}}\left(C_{12}\left(\left\{m_{\tilde{e}_{L I}}, m_{\tilde{e}_{L J}}\right\},|\mu|\right)-C_{23}\left(\left\{m_{\tilde{e}_{L I}}, m_{\tilde{e}_{L J}}\right\},\left|M_{2}\right|\right)\right)\right. \\
& -C_{12}\left(\left\{m_{\tilde{e}_{L I}}, m_{\tilde{e}_{L J}}\right\},|\mu|\right)-C_{23}\left(\left\{m_{\tilde{e}_{L I}}, m_{\tilde{e}_{L J}}\right\},\left|M_{1}\right|\right) \\
& +\left(\left|M_{2}\right|^{2}+\mu^{\star} M_{2}^{\star} \tan \beta\right) \frac{c_{W}^{2}}{s_{W}^{2}} C_{12}\left(\left\{m_{\tilde{e}_{L I}}, m_{\tilde{e}_{L J}}\right\},\left\{|\mu|,\left|M_{2}\right|\right\}\right) \\
& \left.\left.-\left(\left|M_{1}\right|^{2}+\mu^{\star} M_{1}^{\star} \tan \beta\right) C_{12}\left(\left\{m_{\tilde{e}_{L J}}, m_{\tilde{e}_{L I}}\right\},\left\{|\mu|,\left|M_{1}\right|\right\}\right)\right)\right) \\
\left(F_{\gamma} R R\right)_{N}^{J I}= & \frac{e^{2}}{2 c_{W}^{2}} \bar{M}_{R R}^{I J}\left(M_{1}^{\star} C_{12}\left(\left\{m_{\tilde{e}_{L I}}, m_{\tilde{e}_{R I}}, m_{\tilde{e}_{R J}}\right\},\left|M_{1}\right|\right)\left(M_{L R}^{2}\right)_{I I}\right. \\
& -\frac{v_{1}}{\sqrt{2}} Y_{L}^{I}\left(C_{12}\left(\left\{m_{\tilde{e}_{R I}}, m_{\tilde{e}_{R J}}\right\},|\mu|\right)-2 C_{23}\left(\left\{m_{\tilde{e}_{R I}}, m_{\tilde{e}_{R J}}\right\},\left|M_{1}\right|\right)\right. \\
& \left.\left.+\left(\left|M_{1}\right|^{2}+\mu^{\star} M_{1}^{\star} \tan \beta\right) C_{12}\left(\left\{m_{\tilde{e}_{R I}}, m_{\tilde{e}_{R J}}\right\},\left\{\mu,\left|M_{1}\right|\right\}\right)\right)\right) \\
\left(F_{\gamma} A L R\right)_{N}^{J I}= & -\frac{v_{1}}{v_{2}}\left(F_{\gamma}^{\prime} A L R\right)_{N}^{J I}=\frac{e^{2} v_{1}}{2 \sqrt{2} c_{W}^{2}} \sqrt{\bar{M}_{L R}^{I J}} M_{1}^{\star} C_{12}\left(\left\{m_{\tilde{e}_{L I}}, m_{\tilde{e}_{R J}}\right\},\left|M_{1}\right|\right)
\end{aligned}
$$

\section{E.1.2 Vector couplings}

Loop functions $C_{01}$ and $C_{02}$ appearing in eq. (2.6) scale with the inverse of the squared SUSY scale $M^{2}$. Thus, only LL and RR terms contribute to the MI expanded expressions at the $v^{2} / M^{2}$ order, as LR mass insertions always come with additional $v / M$ powers. The non-vanishing chargino and neutralino contributions are:

$$
\begin{aligned}
\left(V_{\gamma L L L}\right)_{C}^{J I} & =\frac{e^{2}}{s_{W}^{2}} \bar{M}_{L L}^{I J} C_{01}\left(\left|M_{2}\right|,\left\{m_{\tilde{\nu}_{I}}, m_{\tilde{\nu}_{J}}\right\}\right) \\
\left(V_{\gamma L L L}\right)_{N}^{J I} & =-\frac{e^{2}}{2 s_{W}^{2} c_{W}^{2}} \bar{M}_{L L}^{I J}\left(c_{W}^{2} C_{02}\left(\left|M_{2}\right|,\left\{m_{\tilde{e}_{L I}}, m_{\tilde{e}_{L J}}\right\}\right)+s_{W}^{2} C_{02}\left(\left|M_{1}\right|,\left\{m_{\tilde{e}_{L I}}, m_{\tilde{e}_{L J}}\right\}\right)\right. \\
\left(V_{\gamma R} R R\right)_{N}^{J I} & =-\frac{2 e^{2}}{c_{W}^{2}} \bar{M}_{R R}^{I J} C_{02}\left(\left|M_{1}\right|,\left\{m_{\tilde{e}_{R I}}, m_{\tilde{e}_{R J}}\right\}\right)
\end{aligned}
$$




\section{E.2 Lepton- $Z^{0}$ vertex}

The leading $v^{2} / M_{\text {SUSY }}^{2}$ terms in the effective $Z \bar{\ell}^{I} \ell^{J}$ vertex defined in eq. (2.7), expanded to the 1st order in LFV mass insertions, depend on divided differences of scalar $C_{0}$ and $C_{2} 3$ point functions. They can be expressed as higher point 1-loop functions (see appendices B and C). We give here the expressions using explicitly scalar 4-, 5- and 6-point functions $D$, $E$ and $F$.

The only non-negligible chargino contribution to $Z \ell \ell^{\prime}$ vertex read:

$$
\begin{aligned}
\left(F_{Z L L L}\right)_{C}^{J I}= & -\frac{e^{5}}{4 s_{W}^{5} c_{W}} \bar{M}_{L L}^{I J}\left(v_{2}^{2} D_{0}\left(\left|M_{2}\right|,|\mu|, m_{\tilde{\nu}^{I}}, m_{\tilde{\nu}^{J}}\right)\right. \\
& +\left(v_{1}^{2}-v_{2}^{2}\right) E_{2}\left(\left|M_{2}\right|,\left|M_{2}\right|,|\mu|, m_{\tilde{\nu}^{I}}, m_{\tilde{\nu}^{J}}\right) \\
& \left.+\frac{1}{2}\left|v_{2} M_{2}+v_{1} \mu^{*}\right|^{2} F_{2}\left(\left|M_{2}\right|,\left|M_{2}\right|,|\mu|,|\mu|, m_{\tilde{\nu}^{I}}, m_{\tilde{\nu}^{J}}\right)\right)
\end{aligned}
$$

Neutralino contributions have a more complicated form. They can be written down as:

$$
\begin{aligned}
\left(F_{Z L L L}\right)_{N}^{J I} & =\frac{e^{3} \sqrt{2}}{16 s_{W}^{3} c_{W}^{3}} \bar{M}_{L L}^{I J}\left(X_{Z N L 4}^{J I}+X_{Z N L 5}^{J I}+X_{Z N L 5}^{I J *}\right) \\
\left(F_{Z R} L L\right)_{N}^{J I} & =\frac{e^{3} \sqrt{2}}{8 s_{W} c_{W}^{3}} \bar{M}_{L L}^{I J}\left(X_{Z N R 4}^{I J}+X_{Z N R 5}^{J I}+X_{Z N R 5}^{I J *}\right) \\
\left(F_{Z L R R}\right)_{N}^{J I} & =\frac{e^{3} \sqrt{2}}{16 s_{W}^{3} c_{W}^{3}} \bar{M}_{R R}^{I J}\left(X_{Z N L 2}^{J I}+X_{Z N L 3}^{J I}+X_{Z N L 3}^{I J *}\right) \\
\left(F_{Z R} R R\right)_{N}^{J I} & =\frac{e^{3} \sqrt{2}}{8 s_{W} c_{W}^{3}} \bar{M}_{R R}^{I J}\left(X_{Z N R 2}^{J I}+X_{Z N R 3}^{J I}+X_{Z N R 3}^{I J *}\right) \\
\left(F_{Z L A L R}\right)_{N}^{J I} & =\left(F_{Z L B L R}\right)_{N}^{I J *}=-\frac{v_{1}}{v_{2}}\left(F_{Z L A L R}^{\prime}\right)_{N}^{J I}=-\frac{v_{1}}{v_{2}}\left(F_{Z L B L R}\right)_{N}^{I J *} \\
& =\frac{e^{3} v_{1}}{16 s_{W}^{3} c_{W}^{3}} \sqrt{\bar{M}_{L R}^{I J}} X_{Z N L 1}^{J I} \\
\left(F_{Z R} A L R\right)_{N}^{J I} & =\left(F_{Z R} B L R\right)_{N}^{I J *}=-\frac{v_{1}}{v_{2}}\left(F_{Z R A L R}^{\prime}\right)_{N}^{J I}=-\frac{v_{1}}{v_{2}}\left(F_{Z R} B L R\right)_{N}^{I J *} \\
& =\frac{e^{3} v_{1}}{4 s_{W} c_{W}^{3}} \sqrt{\bar{M}_{L R}^{I J}} X_{Z N R 1}^{J I}
\end{aligned}
$$

where we defined

$$
\begin{aligned}
X_{Z N L 1}^{J I}= & \sqrt{2}\left(s_{W}^{2} E_{2}\left(\left|M_{1}\right|, m_{\tilde{e}_{L J}}, m_{\tilde{e}_{L I}}, m_{\tilde{e}_{R J}}, m_{\tilde{e}_{R J}}\right)\right. \\
& \left.+c_{W}^{2} E_{2}\left(\left|M_{2}\right|, m_{\tilde{e}_{L J}}, m_{\tilde{e}_{L I}}, m_{\tilde{e}_{R J}}, m_{\tilde{e}_{R J}}\right)\right)\left(M_{L R}^{2}\right)_{J J}^{*} \\
& +Y_{l}^{J}\left(2 v_{1}\left(M_{1}^{*} s_{W}^{2} D_{0}\left(\left|M_{1}\right|,|\mu|, m_{\tilde{e}_{L I}}, m_{\tilde{e}_{R J}}\right)-c_{W}^{2} M_{2}^{*} D_{0}\left(\left|M_{2}\right|,|\mu|, m_{\tilde{e}_{L I}}, m_{\tilde{e}_{R J}}\right)\right)\right. \\
& -s_{W}^{2}\left(v_{1} M_{1}^{*}+v_{2} \mu\right)\left(E_{2}\left(\left|M_{1}\right|,|\mu|, m_{\tilde{e}_{L I}}, m_{\tilde{e}_{R J}}, m_{\tilde{e}_{R J}}\right)\right. \\
& \left.+E_{2}\left(\left|M_{1}\right|,|\mu|,|\mu|, m_{\tilde{e}_{L I}}, m_{\tilde{e}_{R J}}\right)\right) \\
& +c_{W}^{2}\left(v_{1} M_{2}^{*}+v_{2} \mu\right)\left(E_{2}\left(\left|M_{2}\right|,|\mu|, m_{\tilde{e}_{L I}}, m_{\tilde{e}_{R J}}, m_{\tilde{e}_{R J}}\right)\right. \\
& \left.\left.+E_{2}\left(\left|M_{2}\right|,|\mu|,|\mu|, m_{\tilde{e}_{L I}}, m_{\tilde{e}_{R J}}\right)\right)\right)
\end{aligned}
$$




$$
\begin{aligned}
& X_{Z N L 2}^{J I}=\sqrt{2}\left(M_{L R}^{2}\right)_{J J}^{*}\left(M_{L R}^{2}\right)_{I I}\left(s _ { W } ^ { 2 } \left(F_{2}\left(\left|M_{1}\right|, m_{\tilde{e}_{L J}}, m_{\tilde{e}_{L I}}, m_{\tilde{e}_{R J}}, m_{\tilde{e}_{R J}}, m_{\tilde{e}_{R I}}\right)\right.\right. \\
& \left.+F_{2}\left(\left|M_{1}\right|, m_{\tilde{e}_{L J}}, m_{\tilde{e}_{L I}}, m_{\tilde{e}_{R J}}, m_{\tilde{e}_{R I}}, m_{\tilde{e}_{R I}}\right)\right) \\
& +c_{W}^{2}\left(F_{2}\left(\left|M_{2}\right|, m_{\tilde{e}_{L J}}, m_{\tilde{e}_{L I}}, m_{\tilde{e}_{R J}}, m_{\tilde{e}_{R J}}, m_{\tilde{e}_{R I}}\right)\right. \\
& \left.\left.+F_{2}\left(\left|M_{2}\right|, m_{\tilde{e}_{L J}}, m_{\tilde{e}_{L I}}, m_{\tilde{e}_{R J}}, m_{\tilde{e}_{R I}}, m_{\tilde{e}_{R I}}\right)\right)\right) \\
& X_{Z N L 3}^{J I}=Y_{l}^{I *}\left(M_{L R}^{2}\right)_{J J}^{*}\left(2 v _ { 1 } \left(M_{1}^{*} s_{W}^{2} E_{0}\left(\left|M_{1}\right|,|\mu|, m_{\tilde{e}_{L J}}, m_{\tilde{e}_{R J}}, m_{\tilde{e}_{R I}}\right)\right.\right. \\
& \left.-c_{W}^{2} M_{2}^{*} E_{0}\left(\left|M_{2}\right|,|\mu|, m_{\tilde{e}_{L J}}, m_{\tilde{e}_{R J}}, m_{\tilde{e}_{R I}}\right)\right) \\
& -s_{W}^{2}\left(v_{1} M_{1}^{*}+v_{2} \mu\right)\left(F_{2}\left(\left|M_{1}\right|,|\mu|, m_{\tilde{e}_{L J}}, m_{\tilde{e}_{R J}}, m_{\tilde{e}_{R J}}, m_{\tilde{e}_{R I}}\right)\right. \\
& \left.+F_{2}\left(\left|M_{1}\right|,|\mu|, m_{\tilde{e}_{L J}}, m_{\tilde{e}_{R J}}, m_{\tilde{e}_{R I}}, m_{\tilde{e}_{R I}}\right)+F_{2}\left(\left|M_{1}\right|,|\mu|,|\mu|, m_{\tilde{e}_{L J}}, m_{\tilde{e}_{R J}}, m_{\tilde{e}_{R I}}\right)\right) \\
& +c_{W}^{2}\left(v_{1} M_{2}^{*}+v_{2} \mu\right)\left(F_{2}\left(\left|M_{2}\right|,|\mu|, m_{\tilde{e}_{L J}}, m_{\tilde{e}_{R J}}, m_{\tilde{e}_{R J}}, m_{\tilde{e}_{R I}}\right)\right. \\
& \left.\left.+F_{2}\left(\left|M_{2}\right|,|\mu|, m_{\tilde{e}_{L J}}, m_{\tilde{e}_{R J}}, m_{\tilde{e}_{R I}}, m_{\tilde{e}_{R I}}\right)+F_{2}\left(\left|M_{2}\right|,|\mu|,|\mu|, m_{\tilde{e}_{L J}}, m_{\tilde{e}_{R J}}, m_{\tilde{e}_{R I}}\right)\right)\right) \\
& X_{Z N L 4}^{J I}=\frac{e^{2}\left(v_{1}^{2}-v_{2}^{2}\right)}{\sqrt{2} s_{W}^{2} c_{W}^{2}}\left(s_{W}^{4} D_{0}\left(\left|M_{1}\right|,|\mu|, m_{\tilde{e}_{L J}}, m_{\tilde{e}_{L I}}\right)+c_{W}^{4} D_{0}\left(\left|M_{2}\right|,|\mu|, m_{\tilde{e}_{L J}}, m_{\tilde{e}_{L I}}\right)\right. \\
& +2 s_{W}^{2} c_{W}^{2} \operatorname{Re}\left(M_{1} M_{2}^{*}\right) E_{0}\left(\left|M_{1}\right|,\left|M_{2}\right|,|\mu|, m_{\tilde{e}_{L J}}, m_{\tilde{e}_{L I}}\right) \\
& -s_{W}^{4}\left(E_{2}\left(\left|M_{1}\right|,\left|M_{1}\right|,|\mu|, m_{\tilde{e}_{L J}}, m_{\tilde{e}_{L I}}\right)+E_{2}\left(\left|M_{1}\right|,|\mu|,|\mu|, m_{\tilde{e}_{L J}}, m_{\tilde{e}_{L I}}\right)\right) \\
& -c_{W}^{4}\left(E_{2}\left(\left|M_{2}\right|,\left|M_{2}\right|,|\mu|, m_{\tilde{e}_{L J}}, m_{\tilde{e}_{L I}}\right)+E_{2}\left(\left|M_{2}\right|,|\mu|,|\mu|, m_{\tilde{e}_{L J}}, m_{\tilde{e}_{L I}}\right)\right) \\
& +2 s_{W}^{2} c_{W}^{2} E_{2}\left(\left|M_{1}\right|,\left|M_{2}\right|,|\mu|, m_{\tilde{e}_{L J}}, m_{\tilde{e}_{L I}}\right) \\
& +\frac{1}{2} s_{W}^{4}\left(|\mu|^{2}-\left|M_{1}\right|^{2}\right) F_{2}\left(\left|M_{1}\right|,\left|M_{1}\right|,|\mu|,|\mu|, m_{\tilde{e}_{L J}}, m_{\tilde{e}_{L I}}\right) \\
& +\frac{1}{2} c_{W}^{4}\left(|\mu|^{2}-\left|M_{2}\right|^{2}\right) F_{2}\left(\left|M_{2}\right|,\left|M_{2}\right|,|\mu|,|\mu|, m_{\tilde{e}_{L J}}, m_{\tilde{e}_{L I}}\right) \\
& \left.+s_{W}^{2} c_{W}^{2}\left(|\mu|^{2}-\operatorname{Re}\left(M_{1} M_{2}^{*}\right)\right) F_{2}\left(\left|M_{1}\right|,\left|M_{2}\right|,|\mu|,|\mu|, m_{\tilde{e}_{L J}}, m_{\tilde{e}_{L I}}\right)\right) \\
& X_{Z N L 5}^{J I}=Y_{l}^{I *}\left(M_{L R}^{2}\right)_{I I}^{*}\left(2 v _ { 1 } \left(s_{W}^{2} M_{1}^{*} E_{0}\left(\left|M_{1}\right|,|\mu|, m_{\tilde{e}_{L J}}, m_{\tilde{e}_{L I}}, m_{\tilde{e}_{R I}}\right)\right.\right. \\
& \left.-c_{W}^{2} M_{2}^{*} E_{0}\left(\left|M_{2}\right|,|\mu|, m_{\tilde{e}_{L J}}, m_{\tilde{e}_{L I}}, m_{\tilde{e}_{R I}}\right)\right) \\
& -s_{W}^{2}\left(v_{1} M_{1}^{*}+v_{2} \mu\right)\left(F_{2}\left(\left|M_{1}\right|,|\mu|, m_{\tilde{e}_{L J}}, m_{\tilde{e}_{L I}}, m_{\tilde{e}_{R I}}, m_{\tilde{e}_{R I}}\right)\right. \\
& \left.+F_{2}\left(\left|M_{1}\right|,|\mu|,|\mu|, m_{\tilde{e}_{L J}}, m_{\tilde{e}_{L I}}, m_{\tilde{e}_{R I}}\right)\right) \\
& +c_{W}^{2}\left(v_{1} M_{2}^{*}+v_{2} \mu\right)\left(F_{2}\left(\left|M_{2}\right|,|\mu|, m_{\tilde{e}_{L J}}, m_{\tilde{e}_{L I}}, m_{\tilde{e}_{R I}}, m_{\tilde{e}_{R I}}\right)\right. \\
& \left.\left.+F_{2}\left(\left|M_{2}\right|,|\mu|,|\mu|, m_{\tilde{e}_{L J}}, m_{\tilde{e}_{L I}}, m_{\tilde{e}_{R I}}\right)\right)\right) \\
& +\sqrt{2}\left(s_{W}^{2} F_{2}\left(\left|M_{1}\right|, m_{\tilde{e}_{L J}}, m_{\tilde{e}_{L I}}, m_{\tilde{e}_{L I}}, m_{\tilde{e}_{R I}}, m_{\tilde{e}_{R I}}\right)\right. \\
& \left.+c_{W}^{2} F_{2}\left(\left|M_{2}\right|, m_{\tilde{e}_{L J}}, m_{\tilde{e}_{L I}}, m_{\tilde{e}_{L I}}, m_{\tilde{e}_{R I}}, m_{\tilde{e}_{R I}}\right)\right)\left|\left(M_{L R}^{2}\right)_{I I}\right|^{2} \\
& X_{Z N R 1}^{J I}=Y_{l}^{I}\left(2 v_{1} M_{1}^{*} D_{0}\left(\left|M_{1}\right|,|\mu|, m_{\tilde{e}_{L I}}, m_{\tilde{e}_{R J}}\right)\right. \\
& -\left(v_{1} M_{1}^{*}+v_{2} \mu\right)\left(E_{2}\left(\left|M_{1}\right|,|\mu|, m_{\tilde{e}_{L I}}, m_{\tilde{e}_{L I}}, m_{\tilde{e}_{R J}}\right)\right. \\
& \left.\left.+E_{2}\left(\left|M_{1}\right|,|\mu|,|\mu|, m_{\tilde{e}_{L I}}, m_{\tilde{e}_{R J}}\right)\right)\right) \\
& -2 \sqrt{2} E_{2}\left(\left|M_{1}\right|, m_{\tilde{e}_{L I}}, m_{\tilde{e}_{L I}}, m_{\tilde{e}_{R J}}, m_{\tilde{e}_{R I}}\right)\left(M_{L R}^{2}\right)_{I I}^{*}
\end{aligned}
$$




$$
\begin{aligned}
X_{Z N R 2}^{J I}= & \frac{\sqrt{2} e^{2}\left(v_{1}^{2}-v_{2}^{2}\right)}{c_{W}^{2}}\left(\left|M_{1}\right|^{2} E_{0}\left(\left|M_{1}\right|,|\mu|,|\mu|, m_{\tilde{e}_{R J}}, m_{\tilde{e}_{R I}}\right)\right. \\
& +E_{2}\left(\left|M_{1}\right|,\left|M_{1}\right|,|\mu|, m_{\tilde{e}_{R J}}, m_{\tilde{e}_{R I}}\right) \\
& -\frac{1}{2}\left(\left|M_{1}\right|^{2}-|\mu|^{2}\right)\left(F_{2}\left(\left|M_{1}\right|,|\mu|,|\mu|, m_{\tilde{e}_{R J}}, m_{\tilde{e}_{R J}}, m_{\tilde{e}_{R I}}\right)\right. \\
& +F_{2}\left(\left|M_{1}\right|,|\mu|,|\mu|, m_{\tilde{e}_{R J}}, m_{\tilde{e}_{R I}}, m_{\tilde{e}_{R I}}\right) \\
& \left.\left.+2 F_{2}\left(\left|M_{1}\right|,|\mu|,|\mu|,|\mu|, m_{\tilde{e}_{R J}}, m_{\tilde{e}_{R I}}\right)\right)\right) \\
X_{Z N R 3}^{J I}= & Y_{l}^{I}\left(M_{L R}^{2}\right)_{I I}\left(2 v_{1} M_{1}^{*} E_{0}\left(\left|M_{1}\right|,|\mu|, m_{\tilde{e}_{L I}}, m_{\tilde{e}_{R J}}, m_{\tilde{e}_{R I}}\right)\right. \\
& -\left(v_{1} M_{1}^{*}+v_{2} \mu\right)\left(F_{2}\left(\left|M_{1}\right|,|\mu|, m_{\tilde{e}_{L I}}, m_{\tilde{e}_{L I}}, m_{\tilde{e}_{R J}}, m_{\tilde{e}_{R I}}\right)\right. \\
& \left.\left.+F_{2}\left(\left|M_{1}\right|,|\mu|,|\mu|, m_{\tilde{e}_{L I}}, m_{\tilde{e}_{R J}}, m_{\tilde{e}_{R I}}\right)\right)\right) \\
& -2 \sqrt{2} F_{2}\left(\left|M_{1}\right|, m_{\tilde{e}_{L I}}, m_{\tilde{e}_{L I}}, m_{\tilde{e}_{R J}}, m_{\tilde{e}_{R I}}, m_{\tilde{e}_{R I}}\right)\left|\left(M_{L R}^{2}\right)_{I I}\right|^{2} \\
X_{Z N R 4}^{J I}= & -2 \sqrt{2}\left(F_{2}\left(\left|M_{1}\right|, m_{\tilde{e}_{L J}}, m_{\tilde{e}_{L J}}, m_{\tilde{e}_{L I}}, m_{\tilde{e}_{R J}}, m_{\tilde{e}_{R I}}\right)\right. \\
& \left.+F_{2}\left(\left|M_{1}\right|, m_{\tilde{e}_{L J}}, m_{\tilde{e}_{L I}}, m_{\tilde{e}_{L I}}, m_{\tilde{e}_{R J}}, m_{\tilde{e}_{R I}}\right)\right)\left(M_{L R}^{2}\right)_{J J}\left(M_{L R}^{2}\right)_{I I}^{*} \\
X_{Z N R 5}^{J I}= & -Y_{l}^{I}\left(M_{L R}^{2}\right)_{J J}\left(2 \mu v_{2} E_{0}\left(\left|M_{1}\right|,|\mu|, m_{\tilde{e}_{L J}}, m_{\tilde{e}_{L I}}, m_{\tilde{e}_{R J}}\right)\right. \\
& -\left(v_{1} M_{1}^{*}+v_{2} \mu\right)\left(F_{2}\left(\left|M_{1}\right|,\left|M_{1}\right|,|\mu|, m_{\tilde{e}_{L J}}, m_{\tilde{e}_{L I}}, m_{\tilde{e}_{R J}}\right)\right. \\
& \left.\left.+F_{2}\left(\left|M_{1}\right|,|\mu|, m_{\tilde{e}_{L J}}, m_{\tilde{e}_{L I}}, m_{\tilde{e}_{R J}}, m_{\tilde{e}_{R J}}\right)\right)\right)
\end{aligned}
$$

\section{E.3 CP-even Higgs-lepton vertex}

The dominant MI terms in the effective CP-even Higgs - lepton couplings (see eq. (2.13)) can be split into four classes,

$$
F_{h}^{I J K}=\frac{1}{(4 \pi)^{2}}\left(F_{h n d}^{I J K}+F_{h Y}^{I J K}+F_{h d e c}^{I J K}+F_{h m}^{I J K}\right)
$$

defined as (below we give the sum of neutralino and chargino contributions, the latter appearing only as single term depending on sneutrino masses in eq. (E.23) and follow notation of eq. (4.2)):

1. Contributions proportional to non-holomorphic $A_{l}^{\prime}$ trilinear terms, ${ }^{10}$ non-decoupling for $M_{\text {SUSY }} \gg v$ :

$$
\begin{aligned}
\left(F_{\text {hnd } A L R}^{\prime}\right)^{I J K} & =\frac{e^{2}\left(v_{1} Z_{R}^{2 K}-v_{2} Z_{R}^{1 K}\right)}{\sqrt{2} c_{W}^{2} v_{1}} \sqrt{\bar{M}_{L R}^{I J}} M_{1}^{\star} C_{0}\left(\left|M_{1}\right|, m_{\tilde{e}_{L I}}, m_{\tilde{e}_{R J}}\right) \\
\left(F_{\text {hnd } L L}\right)^{I J K} & =\frac{e^{2}\left(v_{1} Z_{R}^{2 K}-v_{2} Z_{R}^{1 K}\right)}{\sqrt{2} c_{W}^{2} v_{1}} \bar{M}_{L L}^{I J} M_{1}^{\star} D_{0}\left(\left|M_{1}\right|, m_{\tilde{e}_{L I}}, m_{\tilde{e}_{L J}}, m_{\tilde{e}_{R J}}\right) A_{L}^{\prime J J} \\
\left(F_{\text {hnd RR }}\right)^{I J K} & =\frac{e^{2}\left(v_{1} Z_{R}^{2 K}-v_{2} Z_{R}^{1 K}\right)}{\sqrt{2} c_{W}^{2} v_{1}} \bar{M}_{R R}^{I J} M_{1}^{\star} D_{0}\left(\left|M_{1}\right|, m_{\tilde{e}_{L I}}, m_{\tilde{e}_{R I}}, m_{\tilde{e}_{R J}}\right) A_{L}^{\prime I I}
\end{aligned}
$$

\footnotetext{
${ }^{10}$ For comparison with commonly used notation of the Higgs mixing angles, note that $\left(v_{1} Z_{R}^{2 K}-v_{2} Z_{R}^{1 K}\right) / v_{1}=\left\{\begin{array}{l}\sin (\alpha-\beta) / \cos \beta \text { for } K=1 \\ \cos (\alpha-\beta) / \cos \beta \text { for } K=2\end{array}\right.$.
} 
2. Contributions suppressed by the lepton Yukawa couplings, also non-decoupling for $M_{\mathrm{SUSY}} \gg v$ :

$$
\begin{aligned}
\left(F_{h Y L L}\right)^{I J K}= & -\frac{e^{2}}{2 \sqrt{2} v_{1} c_{W}^{2} s_{W}^{2}}\left(v_{1} Z_{R}^{2 K}-v_{2} Z_{R}^{1 K}\right)\left(s _ { W } ^ { 2 } M _ { 1 } ^ { \star } \mu ^ { \star } \left(D_{0}\left(\left|M_{1}\right|,|\mu|, m_{\tilde{e}_{L I}}, m_{\tilde{e}_{L J}}\right)\right.\right. \\
& \left.+2 D_{0}\left(\left|M_{1}\right|, m_{\tilde{e}_{L I}}, m_{\tilde{e}_{L J}}, m_{\tilde{e}_{R J}}\right)\right)-c_{W}^{2} M_{2}^{\star} \mu^{\star}\left(D_{0}\left(\left|M_{2}\right|,|\mu|, m_{\tilde{e}_{L I}}, m_{\tilde{e}_{L J}}\right)\right. \\
& \left.\left.+2 D_{0}\left(\left|M_{2}\right|,|\mu|, m_{\tilde{\nu}_{I}}, m_{\tilde{\nu}_{J}}\right)\right)\right) \bar{M}_{L L}^{I J} Y_{L}^{J} \\
\left(F_{h Y R R}\right)^{I J K}= & -\frac{e^{2}}{\sqrt{2} v_{1} c_{W}^{2}}\left(v_{1} Z_{R}^{2 K}-v_{2} Z_{R}^{1 K}\right) M_{1}^{\star} \mu^{\star}\left(D_{0}\left(\left|M_{1}\right|, m_{\tilde{e}_{L I}}, m_{\tilde{e}_{R I}}, m_{\tilde{e}_{R J}}\right)\right. \\
& \left.-D_{0}\left(\left|M_{1}\right|,|\mu|, m_{\tilde{e}_{R I}}, m_{\tilde{e}_{R J}}\right)\right) \bar{M}_{R R}^{I J} Y_{L}^{I}
\end{aligned}
$$

3. Contributions decoupling as $v^{2} / M_{\mathrm{SUSY}}^{2}$. We neglect here terms proportional to $\Delta_{L L}$, $\Delta_{R R}, \Delta_{L R}^{\prime}$ as they are dominated by non-decoupling contributions listed in points 1) and 2). Only the terms proportional to $\Delta_{L R}^{I J}$ and $\Delta_{R L}^{J I *}$ are generated starting at order $v^{2} / M_{\text {SUSY }}^{2}$. To simplify the expressions, below we also neglect terms additionally suppressed by lepton Yukawa couplings (this approximation becomes inaccurate for large $\mu$ and $\tan \beta \geq 30$, when the diagonal LR elements of the slepton mass matrix proportional to $\mu Y_{l}$ become important).

$$
\begin{aligned}
& \left(F_{h d e c} A L R\right)^{I J K}=\left(\frac { e ^ { 4 } } { 4 v _ { 1 } \sqrt { 2 } c _ { W } ^ { 4 } s _ { W } ^ { 2 } } \left(( v _ { 1 } Z _ { R } ^ { 1 K } - v _ { 2 } Z _ { R } ^ { 2 K } ) M _ { 1 } ^ { * } \left(2 s_{W}^{2} D_{0}\left(\left|M_{1}\right|, m_{\tilde{e}_{L I}}, m_{\tilde{e}_{R J}}, m_{\tilde{e}_{R J}}\right)\right.\right.\right. \\
& \left.-\left(2 s_{W}^{2}-1\right) D_{0}\left(\left|M_{1}\right|, m_{\tilde{e}_{L I}}, m_{\tilde{e}_{L I}}, m_{\tilde{e}_{R J}}\right)\right) \\
& +2\left(v_{1} Z_{R}^{1 K}+v_{2} Z_{R}^{2 K}\right)\left(c_{W}^{2}\left(M_{1}^{*}+M_{2}^{*}\right) E_{2}\left(\left|M_{1}\right|,\left|M_{2}\right|,|\mu|, m_{\tilde{e}_{L I}}, m_{\tilde{e}_{R J}}\right)\right. \\
& \left.-2 s_{W}^{2} M_{1}^{*} E_{2}\left(\left|M_{1}\right|,\left|M_{1}\right|,|\mu|, m_{\tilde{e}_{L I}}, m_{\tilde{e}_{R J}}\right)\right) \\
& +2\left(v_{2} Z_{R}^{1 K}+v_{1} Z_{R}^{2 K}\right)\left(M _ { 1 } ^ { * } \mu ^ { * } \left(c_{W}^{2} M_{2}^{*} E_{0}\left(\left|M_{1}\right|,\left|M_{2}\right|,|\mu|, m_{\tilde{e}_{L I}}, m_{\tilde{e}_{R J}}\right)\right.\right. \\
& \left.-s_{W}^{2} M_{1}^{*} E_{0}\left(\left|M_{1}\right|,\left|M_{1}\right|,|\mu|, m_{\tilde{e}_{L I}}, m_{\tilde{e}_{R J}}\right)\right) \\
& +\mu\left(c_{W}^{2} E_{2}\left(\left|M_{1}\right|,\left|M_{2}\right|,|\mu|, m_{\tilde{e}_{L I}}, m_{\tilde{e}_{R J}}\right)\right. \\
& \left.\left.\left.-s_{W}^{2} E_{2}\left(\left|M_{1}\right|,\left|M_{1}\right|,|\mu|, m_{\tilde{e}_{L I}}, m_{\tilde{e}_{R J}}\right)\right)\right)\right) \\
& -\frac{e^{2} v_{1}^{2}}{\sqrt{2} c_{W}^{2}} Z_{R}^{1 K} M_{1}^{*}\left(\left|A_{l}^{I I}\right|^{2} E_{0}\left(\left|M_{1}\right|, m_{\tilde{e}_{L I}}, m_{\tilde{e}_{L I}}, m_{\tilde{e}_{R J}}, m_{\tilde{e}_{R I}}\right)\right. \\
& \left.\left.+\left|A_{l}^{J J}\right|^{2} E_{0}\left(\left|M_{1}\right|, m_{\tilde{e}_{L J}}, m_{\tilde{e}_{L I}}, m_{\tilde{e}_{R J}}, m_{\tilde{e}_{R J}}\right)\right)\right) \sqrt{\bar{M}_{L R}^{I J}} \\
& \left(F_{h d e c ~ B L R}\right)^{I J K}=-\frac{e^{2} v_{1}^{2}}{\sqrt{2} c_{W}^{2}} Z_{R}^{1 K} M_{1}^{*} E_{0}\left(\left|M_{1}\right|, m_{\tilde{e}_{L J}}, m_{\tilde{e}_{L I}}, m_{\tilde{e}_{R J}}, m_{\tilde{e}_{R I}}\right) A_{l}^{I I} A_{l}^{J J} \sqrt{\bar{M}_{L R}^{J I}}
\end{aligned}
$$

4. Contributions decoupling as $M_{h(H)}^{2} / M_{\text {SUSY }}^{2}$. Here, we do not show numerically small terms suppressed by lepton Yukawa couplings or flavour-diagonal $A$ terms:

$$
\left(F_{h m A L R}\right)^{I J K}=\frac{e^{2} M_{H_{0}^{K}}^{2}}{\sqrt{2} c_{W}^{2}} Z_{R}^{1 K} M_{1}^{*} C_{0}^{\prime}\left(\left|M_{1}\right|, m_{\tilde{e}_{R J}}, m_{\tilde{e}_{L I}}\right) \sqrt{\bar{M}_{L R}^{I J}}
$$

where by $C_{0}^{\prime}$ we denote the derivative of $C_{0}$ over the external Higgs mass, $C_{0}^{\prime}=\frac{\partial C_{0}}{\partial M_{h}^{2}}$ (see eq. (B.7)). 


\section{E.4 CP-odd Higgs-lepton vertex}

For the processes considered in this article, the contribution from the LFV CP-odd Higgslepton vertex can become important only in the case of the three body charged lepton decays and only in the limit of $M_{\mathrm{SUSY}} \gg v$, when photon, $Z^{0}$ and box contributions decouple. Thus, we give here only the dominant non-decoupling terms for this vertex.

$$
F_{A}^{I J}=\frac{1}{(4 \pi)^{2}}\left(F_{A n d}^{I J}+F_{A Y}^{I J}+F_{A m}^{I J}\right) .
$$

As for CP-odd Higgs vertices, we give the sum of the neutralino and chargino contributions, the latter appearing only as single term depending on sneutrino masses in eq. (E.28):

1. Contributions proportional to non-holomorphic $A_{l}^{\prime}$ terms:

$$
\begin{aligned}
\left(F_{\text {And } A L R}^{\prime}\right)^{I J} & =-\frac{i e^{2}}{\sqrt{2} c_{W}^{2} \cos \beta} \sqrt{\bar{M}_{L R}^{I J}} M_{1}^{\star} C_{0}\left(\left|M_{1}\right|, m_{\tilde{e}_{L I}}, m_{\tilde{e}_{R J}}\right) \\
\left(F_{\text {And } L L}\right)^{I J} & =-\frac{i e^{2}}{\sqrt{2} c_{W}^{2} \cos \beta} \bar{M}_{L L}^{I J} M_{1}^{\star} D_{0}\left(\left|M_{1}\right|, m_{\tilde{e}_{L I}}, m_{\tilde{e}_{L J}}, m_{\tilde{e}_{R J}}\right) A_{L}^{\prime J J} \\
\left(F_{\text {And } R R}\right)^{I J} & =-\frac{i e^{2}}{\sqrt{2} c_{W}^{2} \cos \beta} \bar{M}_{R R}^{I J} M_{1}^{\star} D_{0}\left(\left|M_{1}\right|, m_{\tilde{e}_{L I}}, m_{\tilde{e}_{R I}}, m_{\tilde{e}_{R J}}\right) A_{L}^{\prime I I}
\end{aligned}
$$

2. Contributions suppressed by lepton Yukawa couplings:

$$
\begin{aligned}
\left(F_{A Y L L}\right)^{I J}= & \frac{i e^{2}}{2 \sqrt{2} c_{W}^{2} s_{W}^{2} \cos \beta}\left(s _ { W } ^ { 2 } M _ { 1 } ^ { \star } \mu ^ { \star } \left(D_{0}\left(\left|M_{1}\right|,|\mu|, m_{\tilde{e}_{L I}}, m_{\tilde{e}_{L J}}\right)\right.\right. \\
& \left.+2 D_{0}\left(\left|M_{1}\right|, m_{\tilde{e}_{L I}}, m_{\tilde{e}_{L J}}, m_{\tilde{e}_{R J}}\right)\right)-c_{W}^{2} M_{2}^{\star} \mu^{\star}\left(D_{0}\left(\left|M_{2}\right|,|\mu|, m_{\tilde{e}_{L I}}, m_{\tilde{e}_{L J}}\right)\right. \\
& \left.\left.+2 D_{0}\left(\left|M_{2}\right|,|\mu|, m_{\tilde{\nu}_{I}}, m_{\tilde{\nu}_{J}}\right)\right)\right) \bar{M}_{L L}^{I J} Y_{L}^{J} \\
\left(F_{A Y} R R\right)^{I J}= & \frac{i e^{2}}{\sqrt{2} c_{W}^{2} \cos \beta} M_{1}^{\star} \mu^{\star}\left(D_{0}\left(\left|M_{1}\right|, m_{\tilde{e}_{L I}}, m_{\tilde{e}_{R I}}, m_{\tilde{e}_{R J}}\right)\right. \\
& \left.-D_{0}\left(\left|M_{1}\right|,|\mu|, m_{\tilde{e}_{R I}}, m_{\tilde{e}_{R J}}\right)\right) \bar{M}_{R R}^{I J} Y_{L}^{I}
\end{aligned}
$$

3. Contributions proportional to $M_{A}^{2} / M_{\text {SUSY }}^{2}$ (see eq. (B.7) for the definition of $C_{0}^{\prime}$ ). As in eq. (E.25) we do not show numerically small terms suppressed by lepton Yukawa couplings or flavour-diagonal $A$ terms:

$$
\left(F_{A m A L R}\right)^{I J K}=-\frac{i e^{2} M_{A}^{2} \sin \beta}{\sqrt{2} c_{W}^{2}} M_{1}^{*} C_{0}^{\prime}\left(\left|M_{1}\right|, m_{\tilde{e}_{R J}}, m_{\tilde{e}_{L I}}\right) \sqrt{\bar{M}_{L R}^{I J}}
$$

\section{E.5 4-lepton box diagrams}

All genuine box diagram contributions listed in eqs. (D.3)-(D.6) have negative mass dimension and without any cancellations explicitly decouple like $v^{2} / M_{\mathrm{SUSY}}^{2}$. Thus, it is sufficient to expand them only in the lowest order in chargino and neutralino mass insertions. Also the LR slepton mass insertions are always associated with additional factors of $v / M_{\text {SUSY }}$. Thus in the leading $v^{2} / M_{\text {SUSY }}^{2}$ order only LL and RR slepton mass insertion can contribute to formulae for box diagrams. 
Expressions listed below are valid only for $\Delta L=1$ processes, i.e. excluding combinations of indices $I=J, K=L$ or $I=K, J=L$ - for these one would also take into account flavour conserving diagrams. As mentioned in section 3.3, we do not consider MI expanded expressions for exotic $\Delta L=2$ processes.

The chargino diagrams contribute significantly only to the $B_{V L L}$, all other contributions are at least double Yukawa suppressed and very small. The $B_{V L L}$ term is:

$$
\begin{aligned}
(4 \pi)^{2} B_{V L L C}^{J I K L}= & \frac{e^{4}}{4 s_{W}^{4}}\left(E_{2}\left(\left|M_{2}\right|,\left|M_{2}\right|, m_{\tilde{\nu}_{I}}, m_{\tilde{\nu}_{J}}, m_{\tilde{\nu}_{K}}\right)\left(\delta^{K L} \Delta_{L L}^{J I} \bar{M}_{L L}^{I J}+\delta^{J L} \Delta_{L L}^{K I} \bar{M}_{L L}^{I K}\right)\right. \\
& \left.+E_{2}\left(\left|M_{2}\right|,\left|M_{2}\right|, m_{\tilde{\nu}_{J}}, m_{\tilde{\nu}_{K}}, m_{\tilde{\nu}_{L}}\right)\left(\delta^{I K} \Delta_{L L}^{J L} \bar{M}_{L L}^{J L}+\delta^{I J} \Delta_{L L}^{K L} \bar{M}_{L L}^{K L}\right)\right)
\end{aligned}
$$

Contributions arising from neutralino box diagrams, both normal and crossed added together, are listed below in eqs. (E.31)-(E.36). We do not give here formulae for the neutralino contributions to $B_{S L L}, B_{S R R}, B_{T L}$ and $B_{T R}$, as they are also double Yukawa suppressed and small.

$$
\begin{aligned}
& (4 \pi)^{2} B_{V L L N}^{J I K L}=\frac{e^{4}}{16 s_{W}^{4} c_{W}^{4}}\left(\left(\delta^{K L} \Delta_{L L}^{J I} \bar{M}_{L L}^{I J}+\delta^{I K} \Delta_{L L}^{J L} \bar{M}_{L L}^{J L}\right)\right. \\
& \times\left(3 c_{W}^{4} E_{2}\left(\left|M_{2}\right|,\left|M_{2}\right|, m_{\tilde{e}_{L I}}, m_{\tilde{e}_{L J}}, m_{\tilde{e}_{L L}}\right)\right. \\
& +3 s_{W}^{4} E_{2}\left(\left|M_{1}\right|,\left|M_{1}\right|, m_{\tilde{e}_{L I}}, m_{\tilde{e}_{L J}}, m_{\tilde{e}_{L L}}\right) \\
& -2 c_{W}^{4} D_{0}\left(\left|M_{2}\right|, m_{\tilde{e}_{L I}}, m_{\tilde{e}_{L J}}, m_{\tilde{e}_{L L}}\right)-2 s_{W}^{4} D_{0}\left(\left|M_{1}\right|, m_{\tilde{e}_{L I}}, m_{\tilde{e}_{L J}}, m_{\tilde{e}_{L L}}\right) \\
& +4 s_{W}^{2} c_{W}^{2} \operatorname{Re}\left(M_{1} M_{2}^{*}\right) E_{0}\left(\left|M_{1}\right|,\left|M_{2}\right|, m_{\tilde{e}_{L I}}, m_{\tilde{e}_{L J}}, m_{\tilde{e}_{L L}}\right) \\
& \left.+2 s_{W}^{2} c_{W}^{2} E_{2}\left(\left|M_{1}\right|,\left|M_{2}\right|, m_{\tilde{e}_{L I}}, m_{\tilde{e}_{L J}}, m_{\tilde{e}_{L L}}\right)\right) \\
& +\left(\delta^{J L} \Delta_{L L}^{K I} \bar{M}_{L L}^{I K}+\delta^{I J} \Delta_{L L}^{K L} \bar{M}_{L L}^{K L}\right)\left(3 c_{W}^{4} E_{2}\left(\left|M_{2}\right|,\left|M_{2}\right|, m_{\tilde{e}_{L I}}, m_{\tilde{e}_{L K}}, m_{\tilde{e}_{L L}}\right)\right. \\
& +3 s_{W}^{4} E_{2}\left(\left|M_{1}\right|,\left|M_{1}\right|, m_{\tilde{e}_{L I}}, m_{\tilde{e}_{L K}}, m_{\tilde{e}_{L L}}\right) \\
& -2 c_{W}^{4} D_{0}\left(\left|M_{2}\right|, m_{\tilde{e}_{L I}}, m_{\tilde{e}_{L K}}, m_{\tilde{e}_{L L}}\right)-2 s_{W}^{4} D_{0}\left(\left|M_{1}\right|, m_{\tilde{e}_{L I}}, m_{\tilde{e}_{L K}}, m_{\tilde{e}_{L L}}\right) \\
& +4 s_{W}^{2} c_{W}^{2} \operatorname{Re}\left(M_{1} M_{2}^{*}\right) E_{0}\left(\left|M_{1}\right|,\left|M_{2}\right|, m_{\tilde{e}_{L I}}, m_{\tilde{e}_{L K}}, m_{\tilde{e}_{L L}}\right) \\
& \left.\left.+2 s_{W}^{2} c_{W}^{2} E_{2}\left(\left|M_{1}\right|,\left|M_{2}\right|, m_{\tilde{e}_{L I}}, m_{\tilde{e}_{L K}}, m_{\tilde{e}_{L L}}\right)\right)\right) \\
& (4 \pi)^{2} B_{V R R N}^{J I K L}=-\frac{e^{4}}{c_{W}^{4}}\left(( \delta ^ { K L } \Delta _ { R R } ^ { I J } \overline { M } _ { R R } ^ { I J } + \delta ^ { I K } \Delta _ { R R } ^ { L J } \overline { M } _ { R R } ^ { J L } ) \left(2 D_{0}\left(\left|M_{1}\right|, m_{\tilde{e}_{R I}}, m_{\tilde{e}_{R J}}, m_{\tilde{e}_{R L}}\right)\right.\right. \\
& \left.-3 E_{2}\left(\left|M_{1}\right|,\left|M_{1}\right|, m_{\tilde{e}_{R I}}, m_{\tilde{e}_{R J}}, m_{\tilde{e}_{R L}}\right)\right) \\
& +\left(\delta^{J L} \Delta_{R R}^{I K} \bar{M}_{R R}^{I K}+. \delta^{I J} \Delta_{R R}^{L K} \bar{M}_{R R}^{K L}\right)\left(2 D_{0}\left(\left|M_{1}\right|, m_{\tilde{e}_{R I}}, m_{\tilde{e}_{R K}}, m_{\tilde{e}_{R L}}\right)\right. \\
& \left.\left.-3 E_{2}\left(\left|M_{1}\right|,\left|M_{1}\right|, m_{\tilde{e}_{R I}}, m_{\tilde{e}_{R K}}, m_{\tilde{e}_{R L}}\right)\right)\right) \\
& (4 \pi)^{2} B_{V L R N}^{J I K L}=-\frac{e^{4}}{4 c_{W}^{4}}\left(\delta ^ { K L } \Delta _ { L L } ^ { J I } \overline { M } _ { L L } ^ { I J } \left(2 D_{0}\left(\left|M_{1}\right|, m_{\tilde{e}_{L I}}, m_{\tilde{e}_{L J}}, m_{\tilde{e}_{R L}}\right)\right.\right. \\
& \left.-3 E_{2}\left(\left|M_{1}\right|,\left|M_{1}\right|, m_{\tilde{e}_{L I}}, m_{\tilde{e}_{L J}}, m_{\tilde{e}_{R L}}\right)\right) \\
& +\delta^{I J} \Delta_{R R}^{L K} \bar{M}_{R R}^{K L}\left(2 D_{0}\left(\left|M_{1}\right|, m_{\tilde{e}_{L I}}, m_{\tilde{e}_{R K}}, m_{\tilde{e}_{R L}}\right)\right. \\
& \left.-3 E_{0}\left(\left|M_{1}\right|,\left|M_{1}\right|, m_{\tilde{e}_{L I}}, m_{\tilde{e}_{R K}}, m_{\tilde{e}_{R L}}\right)\right)
\end{aligned}
$$




$$
\begin{aligned}
(4 \pi)^{2} B_{V R L N}^{J I K L}= & -\frac{e^{4}}{4 c_{W}^{4}}\left(\delta ^ { K L } \Delta _ { R R } ^ { I J } \overline { M } _ { R R } ^ { I J } \left(2 D_{0}\left(\left|M_{1}\right|, m_{\tilde{e}_{R I}}, m_{\tilde{e}_{R J}}, m_{\tilde{e}_{L L}}\right)\right.\right. \\
& \left.-3 E_{2}\left(\left|M_{1}\right|,\left|M_{1}\right|, m_{\tilde{e}_{R I}}, m_{\tilde{e}_{R J}}, m_{\tilde{e}_{L L}}\right)\right) \\
& +\delta^{I J} \Delta_{L L}^{K L} \bar{M}_{L L}^{K L}\left(2 D_{0}\left(\left|M_{1}\right|, m_{\tilde{e}_{R I}}, m_{\tilde{e}_{L K}}, m_{\tilde{e}_{L L}}\right)\right. \\
& \left.-3 E_{0}\left(\left|M_{1}\right|,\left|M_{1}\right|, m_{\tilde{e}_{R I}}, m_{\tilde{e}_{L K}}, m_{\tilde{e}_{L L}}\right)\right) \\
(4 \pi)^{2} B_{S L R N}^{J I K L}= & \frac{e^{4}}{2 c_{W}^{4}}\left(\delta ^ { J L } \Delta _ { L L } ^ { K I } \overline { M } _ { L L } ^ { I K } \left(2 D_{0}\left(\left|M_{1}\right|, m_{\tilde{e}_{L I}}, m_{\tilde{e}_{L K}}, m_{\tilde{e}_{R L}}\right)\right.\right. \\
& \left.-3 E_{2}\left(\left|M_{1}\right|,\left|M_{1}\right|, m_{\tilde{e}_{L I}}, m_{\tilde{e}_{L K}}, m_{\tilde{e}_{R L}}\right)\right) \\
& +\delta^{I K} \Delta_{R R}^{L J} \bar{M}_{R R}^{L J}\left(2 D_{0}\left(\left|M_{1}\right|, m_{\tilde{e}_{L I}}, m_{\tilde{e}_{R J}}, m_{\tilde{e}_{R L}}\right)\right. \\
& \left.\left.-3 E_{2}\left(\left|M_{1}\right|,\left|M_{1}\right|, m_{\tilde{e}_{L I}}, m_{\tilde{e}_{R J}}, m_{\tilde{e}_{R L}}\right)\right)\right) \\
(4 \pi)^{2} B_{S R L N}^{J I K L}= & \frac{e^{4}}{2 c_{W}^{4}}\left(\delta ^ { J L } \Delta _ { R R } ^ { I K } \overline { M } _ { R R } ^ { I K } \left(2 D_{0}\left(\left|M_{1}\right|, m_{\tilde{e}_{R I}}, m_{\tilde{e}_{R K}}, m_{\tilde{e}_{L L}}\right)\right.\right. \\
& \left.-3 E_{2}\left(\left|M_{1}\right|,\left|M_{1}\right|, m_{\tilde{e}_{R I}}, m_{\tilde{e}_{R K}}, m_{\tilde{e}_{L L}}\right)\right) \\
& +\delta^{I K} \Delta_{L L}^{J L} \bar{M}_{L L}^{L J}\left(2 D_{0}\left(\left|M_{1}\right|, m_{\tilde{e}_{R I}}, m_{\tilde{e}_{L J}}, m_{\tilde{e}_{L L}}\right)\right. \\
& \left.\left.-3 E_{2}\left(\left|M_{1}\right|,\left|M_{1}\right|, m_{\tilde{e}_{R I}}, m_{\tilde{e}_{L J}}, m_{\tilde{e}_{L L}}\right)\right)\right)
\end{aligned}
$$

Open Access. This article is distributed under the terms of the Creative Commons Attribution License (CC-BY 4.0), which permits any use, distribution and reproduction in any medium, provided the original author(s) and source are credited.

\section{References}

[1] ATLAS collaboration, Observation of a new particle in the search for the Standard Model Higgs boson with the ATLAS detector at the LHC, Phys. Lett. B 716 (2012) 1 [arXiv: 1207.7214] [INSPIRE].

[2] CMS collaboration, Observation of a new boson at a mass of $125 \mathrm{GeV}$ with the CMS experiment at the LHC, Phys. Lett. B 716 (2012) 30 [arXiv:1207.7235] [INSPIRE].

[3] F. Borzumati and A. Masiero, Large Muon and electron Number Violations in Supergravity Theories, Phys. Rev. Lett. 57 (1986) 961 [INSPIRE].

[4] J.A. Casas and A. Ibarra, Oscillating neutrinos and $\mu \rightarrow e \gamma$, Nucl. Phys. B 618 (2001) 171 [hep-ph/0103065] [INSPIRE].

[5] I. Masina and C.A. Savoy, Sleptonarium: Constraints on the CP and flavor pattern of scalar lepton masses, Nucl. Phys. B 661 (2003) 365 [hep-ph/0211283] [INSPIRE].

[6] A. Brignole and A. Rossi, Anatomy and phenomenology of mu-tau lepton flavor violation in the MSSM, Nucl. Phys. B 701 (2004) 3 [hep-ph/0404211] [INSPIRE].

[7] P. Paradisi, Constraints on SUSY lepton flavor violation by rare processes, JHEP 10 (2005) 006 [hep-ph/0505046] [INSPIRE].

[8] T. Fukuyama, A. Ilakovac and T. Kikuchi, Lepton flavor violating leptonic/semileptonic decays of charged leptons in the minimal supersymmetric standard model, Eur. Phys. J. C 56 (2008) 125 [hep-ph/0506295] [INSPIRE]. 
[9] P. Paradisi, Higgs-mediated $\tau \rightarrow \mu$ and $\tau \rightarrow e$ transitions in II Higgs doublet model and supersymmetry, JHEP 02 (2006) 050 [hep-ph/0508054] [INSPIRE].

[10] A. Dedes, S. Rimmer and J. Rosiek, Neutrino masses in the lepton number violating MSSM, JHEP 08 (2006) 005 [hep-ph/0603225] [INSPIRE].

[11] A. Dedes, H.E. Haber and J. Rosiek, Seesaw mechanism in the sneutrino sector and its consequences, JHEP 11 (2007) 059 [arXiv:0707.3718] [INSPIRE].

[12] S. Antusch and S.F. King, Lepton Flavour Violation in the Constrained MSSM with Constrained Sequential Dominance, Phys. Lett. B 659 (2008) 640 [arXiv:0709.0666] [INSPIRE].

[13] E. Arganda, M.J. Herrero and J. Portoles, Lepton flavour violating semileptonic tau decays in constrained MSSM-seesaw scenarios, JHEP 06 (2008) 079 [arXiv:0803.2039] [INSPIRE].

[14] A. Ilakovac and A. Pilaftsis, Supersymmetric Lepton Flavour Violation in Low-Scale Seesaw Models, Phys. Rev. D 80 (2009) 091902 [arXiv:0904.2381] [INSPIRE].

[15] L. Calibbi, J. Jones-Perez, A. Masiero, J.-h. Park, W. Porod and O. Vives, FCNC and CP Violation Observables in a SU(3)-flavoured MSSM, Nucl. Phys. B 831 (2010) 26 [arXiv: 0907.4069] [INSPIRE].

[16] W. Altmannshofer, A.J. Buras, S. Gori, P. Paradisi and D.M. Straub, Anatomy and Phenomenology of FCNC and CPV Effects in SUSY Theories, Nucl. Phys. B 830 (2010) 17 [arXiv:0909.1333] [INSPIRE].

[17] L. Calibbi, A. Faccia, A. Masiero and S.K. Vempati, Lepton flavour violation from SUSY-GUTs: Where do we stand for MEG, PRISM/PRIME and a super flavour factory, Phys. Rev. D 74 (2006) 116002 [hep-ph/0605139] [INSPIRE].

[18] L. Calibbi, M. Frigerio, S. Lavignac and A. Romanino, Flavour violation in supersymmetric $\mathrm{SO}(10)$ unification with a type II seesaw mechanism, JHEP 12 (2009) 057 [arXiv:0910.0377] [INSPIRE].

[19] J. Hisano, M. Nagai, P. Paradisi and Y. Shimizu, Waiting for $\mu \rightarrow e \gamma$ from the MEG experiment, JHEP 12 (2009) 030 [arXiv:0904.2080] [INSPIRE].

[20] J. Girrbach, S. Mertens, U. Nierste and S. Wiesenfeldt, Lepton flavour violation in the MSSM, JHEP 05 (2010) 026 [arXiv:0910. 2663] [INSPIRE].

[21] C. Biggio and L. Calibbi, Phenomenology of SUSY SU(5) with type I+III seesaw, JHEP 10 (2010) 037 [arXiv: 1007.3750] [INSPIRE].

[22] J.N. Esteves, J.C. Romao, M. Hirsch, F. Staub and W. Porod, Supersymmetric type-III seesaw: lepton flavour violating decays and dark matter, Phys. Rev. D 83 (2011) 013003 [arXiv: 1010.6000] [INSPIRE].

[23] A. Ilakovac, A. Pilaftsis and L. Popov, Charged lepton flavor violation in supersymmetric low-scale seesaw models, Phys. Rev. D 87 (2013) 053014 [arXiv:1212.5939] [INSPIRE].

[24] M. Arana-Catania, S. Heinemeyer and M.J. Herrero, New Constraints on General Slepton Flavor Mixing, Phys. Rev. D 88 (2013) 015026 [arXiv: 1304.2783] [INSPIRE].

[25] T. Goto, Y. Okada, T. Shindou, M. Tanaka and R. Watanabe, Lepton flavor violation in the supersymmetric seesaw model after the LHC 8 TeV run, Phys. Rev. D 91 (2015) 033007 [arXiv: 1412.2530] [INSPIRE]. 
[26] A. Abada, M.E. Krauss, W. Porod, F. Staub, A. Vicente and C. Weiland, Lepton flavor violation in low-scale seesaw models: SUSY and non-SUSY contributions, JHEP 11 (2014) 048 [arXiv: 1408.0138] [INSPIRE].

[27] A. Vicente, Lepton flavor violation beyond the MSSM, Adv. High Energy Phys. 2015 (2015) 686572 [arXiv: 1503.08622] [INSPIRE].

[28] C. Bonilla, M.E. Krauss, T. Opferkuch and W. Porod, Perspectives for Detecting Lepton Flavour Violation in Left-Right Symmetric Models, JHEP 03 (2017) 027 [arXiv: 1611.07025] [INSPIRE].

[29] M. Lindner, M. Platscher and F.S. Queiroz, A Call for New Physics: The Muon Anomalous Magnetic Moment and Lepton Flavor Violation, Phys. Rept. 731 (2018) 1 [arXiv: 1610.06587] [INSPIRE].

[30] L. Calibbi and G. Signorelli, Charged Lepton Flavour Violation: An Experimental and Theoretical Introduction, Riv. Nuovo Cim. 41 (2018) 1 [arXiv:1709.00294] [INSPIRE].

[31] A. Dedes, M. Paraskevas, J. Rosiek, K. Suxho and K. Tamvakis, Mass Insertions vs. Mass Eigenstates calculations in Flavour Physics, JHEP 06 (2015) 151 [arXiv:1504.00960] [INSPIRE].

[32] J. Rosiek, MassToMI - A Mathematica package for an automatic Mass Insertion expansion, Comput. Phys. Commun. 201 (2016) 144 [arXiv:1509.05030] [INSPIRE].

[33] F. Gabbiani, E. Gabrielli, A. Masiero and L. Silvestrini, A complete analysis of FCNC and CP constraints in general SUSY extensions of the standard model, Nucl. Phys. B 477 (1996) 321 [hep-ph/9604387] [INSPIRE].

[34] M. Misiak, S. Pokorski and J. Rosiek, Supersymmetry and FCNC effects, Adv. Ser. Direct. High Energy Phys. 15 (1998) 795 [hep-ph/9703442] [INSPIRE].

[35] B. de Wit, M.T. Grisaru and M. Roček, Nonholomorphic corrections to the one loop $N=2$ superYang-Mills action, Phys. Lett. B 374 (1996) 297 [hep-th/9601115] [INSPIRE].

[36] M. Matone, Modular invariance and structure of the exact Wilsonian action of $N=2$ supersymmetric Yang-Mills theory, Phys. Rev. Lett. 78 (1997) 1412 [hep-th/9610204] [INSPIRE].

[37] D. Bellisai, F. Fucito, M. Matone and G. Travaglini, Nonholomorphic terms in $N=2$ SUSY Wilsonian actions and the renormalization group equation, Phys. Rev. D 56 (1997) 5218 [hep-th/9706099] [INSPIRE].

[38] M. Dine and N. Seiberg, Comments on higher derivative operators in some SUSY field theories, Phys. Lett. B 409 (1997) 239 [hep-th/9705057] [INSPIRE].

[39] N. Arkani-Hamed and R. Rattazzi, Exact results for nonholomorphic masses in softly broken supersymmetric gauge theories, Phys. Lett. B 454 (1999) 290 [hep-th/9804068] [INSPIRE].

[40] F. Gonzalez-Rey and M. Roček, Nonholomorphic $N=2$ terms in $N=4 S Y M$ : One loop calculation in $N=2$ superspace, Phys. Lett. B 434 (1998) 303 [hep-th/9804010] [INSPIRE].

[41] E.I. Buchbinder, I.L. Buchbinder and S.M. Kuzenko, Nonholomorphic effective potential in $N=4 \mathrm{SU}(N) S Y M$, Phys. Lett. B 446 (1999) 216 [hep-th/9810239] [InSPIRE].

[42] S.P. Martin, Dimensionless supersymmetry breaking couplings, flat directions, and the origin of intermediate mass scales, Phys. Rev. D 61 (2000) 035004 [hep-ph/9907550] [INSPIRE]. 
[43] J. Rosiek, Complete Set of Feynman Rules for the Minimal Supersymmetric Extension of the Standard Model, Phys. Rev. D 41 (1990) 3464 [inSPIRE].

[44] J. Rosiek, Complete set of Feynman rules for the MSSM: Erratum, hep-ph/9511250 [INSPIRE].

[45] B.C. Allanach et al., SUSY Les Houches Accord 2, Comput. Phys. Commun. 180 (2009) 8 [arXiv:0801.0045] [INSPIRE].

[46] S.M. Barr and A. Zee, Electric Dipole Moment of the Electron and of the Neutron, Phys. Rev. Lett. 65 (1990) 21 [Erratum ibid. 65 (1990) 2920] [INSPIRE].

[47] D. Chang, W.S. Hou and W.-Y. Keung, Two loop contributions of flavor changing neutral Higgs bosons to $\mu \rightarrow e \gamma$, Phys. Rev. D 48 (1993) 217 [hep-ph/9302267] [INSPIRE].

[48] J. Hisano, M. Nagai and P. Paradisi, New Two-loop Contributions to Hadronic EDMs in the MSSM, Phys. Lett. B 642 (2006) 510 [hep-ph/0606322] [INSPIRE].

[49] M. Jung and A. Pich, Electric Dipole Moments in Two-Higgs-Doublet Models, JHEP 04 (2014) 076 [arXiv: 1308.6283] [INSPIRE].

[50] T. Abe, J. Hisano, T. Kitahara and K. Tobioka, Gauge invariant Barr-Zee type contributions to fermionic EDMs in the two-Higgs doublet models, JHEP 01 (2014) 106 [Erratum ibid. 04 (2016) 161] [arXiv:1311.4704] [INSPIRE].

[51] V. Ilisie, New Barr-Zee contributions to $(g-2)_{\mu}$ in two-Higgs-doublet models, JHEP 04 (2015) 077 [arXiv: 1502.04199] [INSPIRE].

[52] A. Crivellin, J. Heeck and P. Stoffer, A perturbed lepton-specific two-Higgs-doublet model facing experimental hints for physics beyond the Standard Model, Phys. Rev. Lett. 116 (2016) 081801 [arXiv: 1507.07567] [INSPIRE].

[53] A.J. Buras, P.H. Chankowski, J. Rosiek and L. Slawianowska, $\Delta M_{d, s}, B^{0} d, s \rightarrow \mu^{+} \mu^{-}$and $B \rightarrow X_{s} \gamma$ in supersymmetry at large $\tan \beta$, Nucl. Phys. B 659 (2003) 3 [hep-ph/0210145] [INSPIRE].

[54] A. Crivellin, Effective Higgs Vertices in the generic MSSM, Phys. Rev. D 83 (2011) 056001 [arXiv: 1012.4840] [INSPIRE].

[55] A. Crivellin, L. Hofer and J. Rosiek, Complete resummation of chirally-enhanced loop-effects in the MSSM with non-minimal sources of flavor-violation, JHEP 07 (2011) 017 [arXiv:1103.4272] [INSPIRE].

[56] A. Crivellin and C. Greub, Two-loop supersymmetric QCD corrections to Higgs-quark-quark couplings in the generic MSSM, Phys. Rev. D 87 (2013) 015013 [Erratum ibid. D 87 (2013) 079901] [arXiv:1210.7453] [INSPIRE].

[57] L.J. Hall, R. Rattazzi and U. Sarid, The top quark mass in supersymmetric $\mathrm{SO}(10)$ unification, Phys. Rev. D 50 (1994) 7048 [hep-ph/9306309] [INSPIRE].

[58] M. Carena, M. Olechowski, S. Pokorski and C.E.M. Wagner, Electroweak symmetry breaking and bottom - top Yukawa unification, Nucl. Phys. B 426 (1994) 269 [hep-ph/9402253] [INSPIRE].

[59] M. Carena, D. Garcia, U. Nierste and C.E.M. Wagner, Effective Lagrangian for the $\bar{t} b H^{+}$ interaction in the MSSM and charged Higgs phenomenology, Nucl. Phys. B 577 (2000) 88 [hep-ph/9912516] [INSPIRE]. 
[60] C. Bobeth, T. Ewerth, F. Krüger and J. Urban, Analysis of neutral Higgs boson contributions to the decays $\bar{B}(s) \rightarrow \ell^{+} \ell^{-}$and $\bar{B} \rightarrow K \ell^{+} \ell^{-}$, Phys. Rev. D 64 (2001) 074014 [hep-ph/0104284] [INSPIRE].

[61] K.S. Babu and C.F. Kolda, Higgs mediated $B^{0} \rightarrow \mu^{+} \mu^{-}$in minimal supersymmetry, Phys. Rev. Lett. 84 (2000) 228 [hep-ph/9909476] [INSPIRE].

[62] G. Isidori and A. Retico, Scalar flavor changing neutral currents in the large tan beta limit, JHEP 11 (2001) 001 [hep-ph/0110121] [INSPIRE].

[63] A. Dedes and A. Pilaftsis, Resummed effective Lagrangian for Higgs mediated FCNC interactions in the CP violating MSSM, Phys. Rev. D 67 (2003) 015012 [hep-ph/0209306] [INSPIRE].

[64] L. Hofer, U. Nierste and D. Scherer, Resummation of tan-beta-enhanced supersymmetric loop corrections beyond the decoupling limit, JHEP 10 (2009) 081 [arXiv:0907.5408] [INSPIRE].

[65] D. Noth and M. Spira, Supersymmetric Higgs Yukawa Couplings to Bottom Quarks at next-to-next-to-leading Order, JHEP 06 (2011) 084 [arXiv:1001.1935] [INSPIRE].

[66] J. Rosiek, P. Chankowski, A. Dedes, S. Jager and P. Tanedo, SUSY_FLAVOR: A Computational Tool for FCNC and CP-violating Processes in the MSSM, Comput. Phys. Commun. 181 (2010) 2180 [arXiv:1003.4260] [INSPIRE].

[67] A. Crivellin, J. Rosiek, P.H. Chankowski, A. Dedes, S. Jaeger and P. Tanedo, SUSY_FLAVOR v2: A computational tool for FCNC and CP-violating processes in the MSSM, Comput. Phys. Commun. 184 (2013) 1004 [arXiv:1203.5023] [INSPIRE].

[68] J. Rosiek, SUSY FLAVOR v2.5: a computational tool for FCNC and CP-violating processes in the MSSM, Comput. Phys. Commun. 188 (2015) 208 [arXiv:1410.0606] [INSPIRE].

[69] Particle Data Group collaboration, K.A. Olive et al., Review of Particle Physics, Chin. Phys. C 38 (2014) 090001 [inSPIRE].

[70] S.A.R. Ellis and A. Pierce, Impact of Future Lepton Flavor Violation Measurements in the Minimal Supersymmetric Standard Model, Phys. Rev. D 94 (2016) 015014 [arXiv: 1604.01419] [INSPIRE].

[71] R. Kitano, M. Koike and Y. Okada, Detailed calculation of lepton flavor violating muon electron conversion rate for various nuclei, Phys. Rev. D 66 (2002) 096002 [Erratum ibid. D 76 (2007) 059902] [hep-ph/0203110] [INSPIRE].

[72] A. Crivellin, S. Davidson, G.M. Pruna and A. Signer, Renormalisation-group improved analysis of $\mu \rightarrow e$ processes in a systematic effective-field-theory approach, JHEP 05 (2017) 117 [arXiv: 1702.03020$]$ [INSPIRE].

[73] M.A. Shifman, A.I. Vainshtein and V.I. Zakharov, Remarks on Higgs Boson Interactions with Nucleons, Phys. Lett. B 78 (1978) 443 [INSPIRE].

[74] V. Cirigliano, R. Kitano, Y. Okada and P. Tuzon, On the model discriminating power of $\mu \rightarrow e$ conversion in nuclei, Phys. Rev. D 80 (2009) 013002 [arXiv:0904.0957] [INSPIRE].

[75] A. Crivellin, M. Hoferichter and M. Procura, Accurate evaluation of hadronic uncertainties in spin-independent WIMP-nucleon scattering: Disentangling two- and three-flavor effects, Phys. Rev. D 89 (2014) 054021 [arXiv: 1312.4951] [INSPIRE]. 
[76] A. Crivellin, M. Hoferichter and M. Procura, Improved predictions for $\mu \rightarrow e$ conversion in nuclei and Higgs-induced lepton flavor violation, Phys. Rev. D 89 (2014) 093024 [arXiv: 1404.7134] [INSPIRE].

[77] M. Hoferichter, J. Ruiz de Elvira, B. Kubis and U.-G. Meißner, High-Precision Determination of the Pion-Nucleon $\sigma$ Term from Roy-Steiner Equations, Phys. Rev. Lett. 115 (2015) 092301 [arXiv: 1506.04142] [INSPIRE].

[78] P. Junnarkar and A. Walker-Loud, Scalar strange content of the nucleon from lattice QCD, Phys. Rev. D 87 (2013) 114510 [arXiv:1301.1114] [INSPIRE].

[79] J.M. Alarcon, J. Martin Camalich and J.A. Oller, The chiral representation of the $\pi N$ scattering amplitude and the pion-nucleon sigma term, Phys. Rev. D 85 (2012) 051503 [arXiv:1110.3797] [INSPIRE].

[80] J.M. Alarcon, L.S. Geng, J. Martin Camalich and J.A. Oller, The strangeness content of the nucleon from effective field theory and phenomenology, Phys. Lett. B 730 (2014) 342 [arXiv: 1209.2870] [INSPIRE].

[81] A. Czarnecki, W.J. Marciano and K. Melnikov, Coherent muon electron conversion in muonic atoms, AIP Conf. Proc. 435 (1998) 409 [hep-ph/9801218] [INSPIRE].

[82] T. Suzuki, D.F. Measday and J.P. Roalsvig, Total Nuclear Capture Rates for Negative Muons, Phys. Rev. C 35 (1987) 2212 [InSPIRE].

[83] J. Rosiek, General Mass Insertion Expansion in Flavor Physics, in 5th Large Hadron Collider Physics Conference (LHCP 2017) Shanghai, China, May 15-20, 2017, arXiv: 1708.06818 [INSPIRE].

[84] E. Arganda, M.J. Herrero, R. Morales and A. Szynkman, Analysis of the $h, H, A \rightarrow \tau \mu$ decays induced from SUSY loops within the Mass Insertion Approximation, JHEP 03 (2016) 055 [arXiv: 1510.04685] [INSPIRE].

[85] BABAR collaboration, B. Aubert et al., Searches for Lepton Flavor Violation in the Decays $\tau^{ \pm} \rightarrow e^{ \pm} \gamma$ and $\tau^{ \pm} \rightarrow \mu^{ \pm} \gamma$, Phys. Rev. Lett. 104 (2010) 021802 [arXiv:0908.2381] [INSPIRE].

[86] Belle and Belle-II collaborations, K. Hayasaka, Results and prospects on lepton flavor violation at Belle/Belle II, J. Phys. Conf. Ser. 408 (2013) 012069 [InSPIRE].

[87] G. Cavoto, A. Papa, F. Renga, E. Ripiccini and C. Voena, The quest for $\mu \rightarrow$ e $\gamma$ and its experimental limiting factors at future high intensity muon beams, Eur. Phys. J. C 78 (2018) 37 [arXiv:1707.01805] [INSPIRE].

[88] Belle collaboration, K. Hayasaka et al., New Search for $\tau \rightarrow \mu \gamma$ and $\tau \rightarrow e \gamma$ Decays at Belle, Phys. Lett. B 666 (2008) 16 [arXiv:0705.0650] [INSPIRE].

[89] MEG collaboration, J. Adam et al., New constraint on the existence of the $\mu^{+} \rightarrow e^{+} \gamma$ decay, Phys. Rev. Lett. 110 (2013) 201801 [arXiv:1303.0754] [INSPIRE].

[90] MEG II collaboration, A.M. Baldini et al., The design of the MEG II experiment, arXiv:1801.04688 [INSPIRE].

[91] ATLAS collaboration, Search for the lepton flavor violating decay $Z \rightarrow e \mu$ in pp collisions at $\sqrt{s}=8 \mathrm{TeV}$ with the ATLAS detector, Phys. Rev. D 90 (2014) 072010 [arXiv: 1408.5774] [INSPIRE]. 
[92] DELPHI collaboration, P. Abreu et al., Search for lepton flavor number violating $Z_{0}$ decays, Z. Phys. C 73 (1997) 243 [InSPIRE].

[93] SINDRUM collaboration, U. Bellgardt et al., Search for the Decay $\mu^{+} \rightarrow e^{+} e^{+} e^{-}$, Nucl. Phys. B 299 (1988) 1 [INSPIRE].

[94] A. Blondel et al., Research Proposal for an Experiment to Search for the Decay $\mu \rightarrow$ eee, arXiv: 1301.6113 [INSPIRE].

[95] Mu3e collaboration, N. Berger, The Muße Experiment, Nucl. Phys. Proc. Suppl. 248-250 (2014) 35 [INSPIRE].

[96] K. Hayasaka et al., Search for Lepton Flavor Violating Tau Decays into Three Leptons with 719 Million Produced Tau+Tau- Pairs, Phys. Lett. B 687 (2010) 139 [arXiv:1001.3221] [INSPIRE].

[97] CMS collaboration, Search for lepton flavour violating decays of the Higgs boson to $\mu \tau$ and eT in proton-proton collisions at $\sqrt{s}=13 \mathrm{TeV}$, [arXiv:1712.07173] [INSPIRE].

[98] CMS collaboration, Search for lepton-flavour-violating decays of the Higgs boson to etau and emu at $\sqrt{s}=8 \mathrm{TeV}$, CMS-PAS-HIG-14-040 [INSPIRE].

[99] SINDRUM II collaboration, W.H. Bertl et al., A search for muon to electron conversion in muonic gold, Eur. Phys. J. C 47 (2006) 337 [INSPIRE].

[100] Mu2e collaboration, R.J. Abrams et al., Mu2e Conceptual Design Report, arXiv: 1211.7019 [INSPIRE].

[101] T. Appelquist and J. Carazzone, Infrared Singularities and Massive Fields, Phys. Rev. D 11 (1975) 2856 [INSPIRE].

[102] E. Arganda, A.M. Curiel, M.J. Herrero and D. Temes, Lepton flavor violating Higgs boson decays from massive seesaw neutrinos, Phys. Rev. D 71 (2005) 035011 [hep-ph/0407302] [INSPIRE].

[103] A. Azatov, S. Chang, N. Craig and J. Galloway, Higgs fits preference for suppressed down-type couplings: Implications for supersymmetry, Phys. Rev. D 86 (2012) 075033 [arXiv:1206.1058] [INSPIRE].

[104] C. Petersson, A. Romagnoni and R. Torre, Liberating Higgs couplings in supersymmetry, Phys. Rev. D 87 (2013) 013008 [arXiv:1211.2114] [INSPIRE].

[105] A. Bartl, H. Eberl, E. Ginina, K. Hidaka and W. Majerotto, $h^{0} \rightarrow c \bar{c}$ as a test case for quark flavor violation in the MSSM, Phys. Rev. D 91 (2015) 015007 [arXiv:1411.2840] [INSPIRE].

[106] M. Arana-Catania, E. Arganda and M.J. Herrero, Non-decoupling SUSY in LFV Higgs decays: a window to new physics at the LHC, JHEP 09 (2013) 160 [Erratum ibid. 10 (2015) 192] [arXiv: 1304.3371] [INSPIRE].

[107] D. Aloni, Y. Nir and E. Stamou, Large BR(h $\rightarrow \tau \mu)$ in the MSSM?, JHEP 04 (2016) 162 [arXiv: 1511.00979] [INSPIRE].

[108] G. Barenboim, C. Bosch, J.S. Lee, M.L. López-Ibáñez and O. Vives, Flavor-changing Higgs boson decays into bottom and strange quarks in supersymmetric models, Phys. Rev. D 92 (2015) 095017 [arXiv: 1507.08304] [INSPIRE].

[109] M.E. Gomez, S. Heinemeyer and M. Rehman, Lepton flavor violating Higgs Boson Decays in Supersymmetric High Scale Seesaw Models, arXiv:1703.02229 [INSPIRE]. 
[110] A. Dedes, M. Paraskevas, J. Rosiek, K. Suxho and K. Tamvakis, Rare Top-quark Decays to Higgs boson in MSSM, JHEP 11 (2014) 137 [arXiv:1409.6546] [INSPIRE].

[111] A. Crivellin, A. Kokulu and C. Greub, Flavor-phenomenology of two-Higgs-doublet models with generic Yukawa structure, Phys. Rev. D 87 (2013) 094031 [arXiv:1303.5877] [INSPIRE].

[112] A. Dedes, J. Rosiek and P. Tanedo, Complete One-Loop MSSM Predictions for $B \rightarrow \ell \ell^{\prime}$ at the Tevatron and LHC, Phys. Rev. D 79 (2009) 055006 [arXiv:0812.4320] [inSPIRE]. 